\title{
Lipid Digestion of Oil-in-water Emulsions Stabilized with Low Molecular Weight Surfactants
}

\author{
by \\ Natalie Ng \\ A Thesis \\ presented to \\ The University of Guelph \\ In partial fulfilment of requirements \\ for the degree of \\ Master of Science \\ in \\ Food Science
}

Guelph, Ontario, Canada

(C) Natalie Ng, August, 2019 


\section{ABSTRACT \\ Lipid Digestion of Oil-in-water Emulsions Stabilized with Low Molecular Weight Surfactants}

Natalie Ng

University of Guelph, 2019

\section{Advisor: \\ Dr. Michael Rogers}

Lipid digestion kinetics and fatty acid bioaccessibility were compared between oil-in-water (o/w) emulsions stabilized with monoglycerides (i.e., glycerol monostearate (1-GMS), glycerol monooleate (1-GMO), or sn-2 glycerol monooleate (2-GMO)) or commonly-used synthetic surfactants (i.e., Span 60, Span 80, Tween 60 , or Tween 80) using a multi-compartmental, simulated digestion model (TIM-1). Although statistically significant differences in induction time and rate of lipid digestion were not observed, total bioaccessibility was significantly lower for emulsions stabilized with Span 80 and 2-GMO. Comparable trends were observed between area under the curve (AUC) of the absolute bioaccessibility and total overall bioaccessibility obtained by fitting a three-parameter shifted logistic model to the cumulative bioaccessibility. No significant differences for overall bioaccessibility were observed between oil-in-water emulsions stabilized by commercial 1-GMO and 1-GMS, Span 60, Tween 60 and Tween 80 . The results confirm that $s n$-position and surfactant choice influence the total extent of lipid digestion (total bioaccessibility) in vitro. 


\section{ACKNOWLEDGEMENTS}

I am extremely grateful to have learnt and experienced so much over these past two years, in terms of both personal growth and as a researcher. This was all made possible by my support system of mentors, friends, and family. I would like to begin by thanking my advisor, Dr. Michael Rogers, for his careful supervision and direction that challenged me to be a better researcher, critical thinker, and scientific writer. I would also like to thank my advisory committee members, Dr. Iris Joye and Dr. Doug Goff, for their guidance and useful feedback in overseeing the progression of my project.

I would also like to express gratitude to members of my own lab group, other lab groups, and individuals whom have helped me greatly. Members of my lab group for assistance with countless TIM-1 runs, experiments, and the camaraderie. Members of Dr. Spagnuolo's lab group for always accommodating my usage of their fume hood and nitrogen supply. Saeed, from Dr. Marangoni's lab group, for his genuine willingness to help, positivity, and advice. Sameer, from the NMR center, for all of his patience and help on one of the toughest parts of my project. Tricia, and everyone in the main office, for all their support throughout all my years in the Food Science department as both an undergraduate and graduate student.

Last but not least, I would not have made it thus far without the continued encouragement

and comfort from my friends and family. For bearing with me through the ups and downs, providing much needed moral support, and filling these past two years with so many great memories. 


\section{TABLE OF CONTENTS}

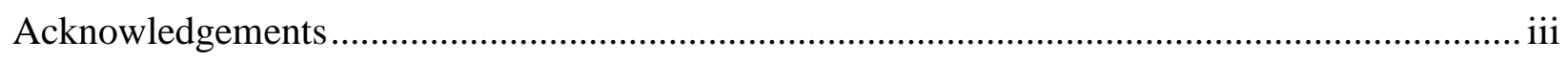

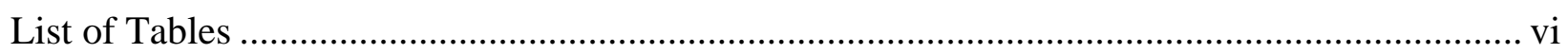

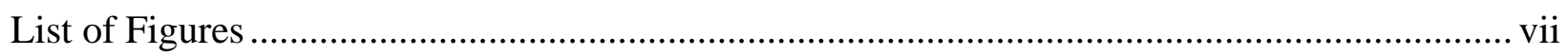

List of Symbols, Abbreviations or Nomenclature ……….................................................. ix

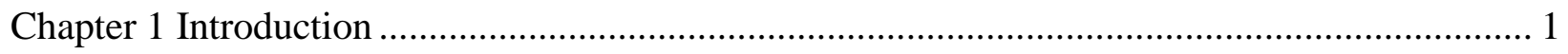

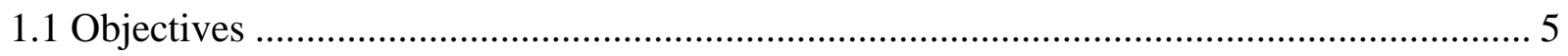

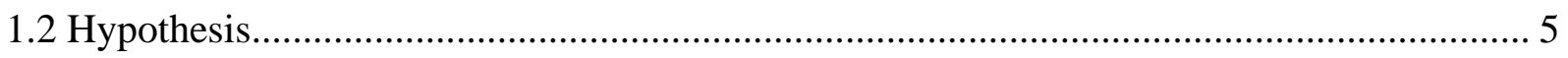

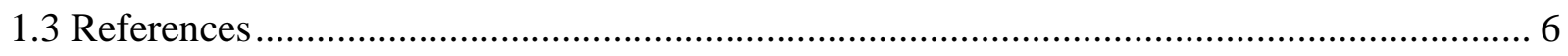

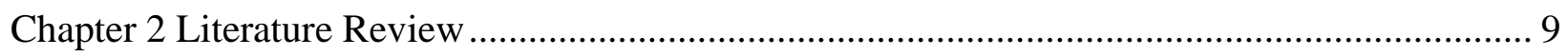

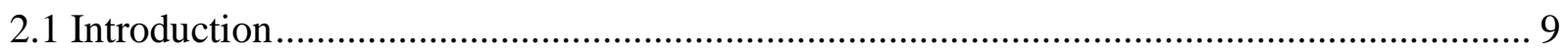

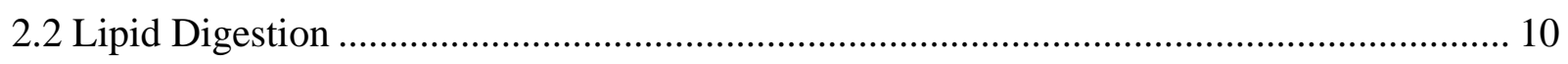

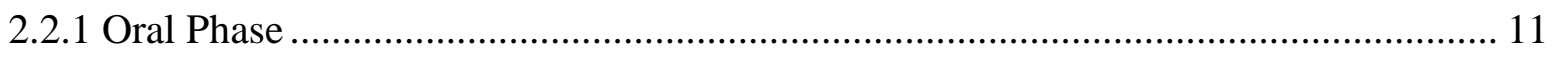

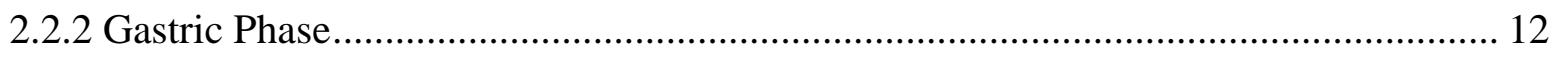

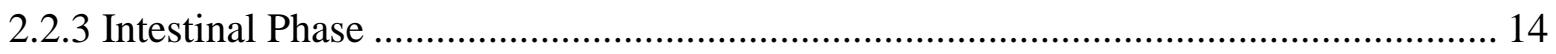

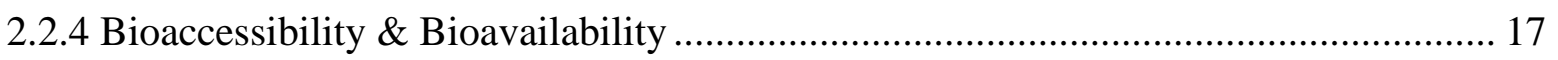

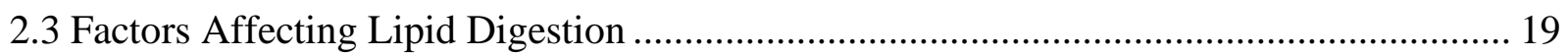

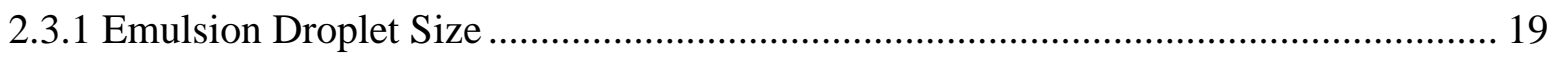

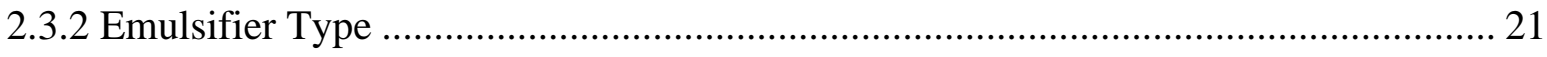

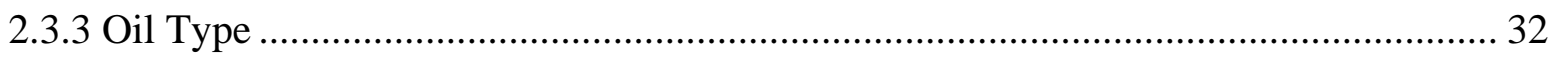

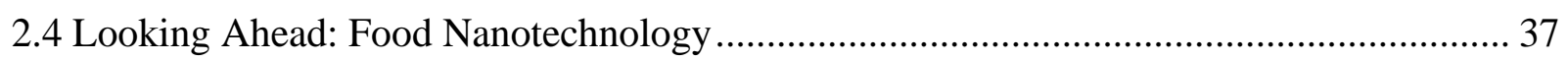

2.4.1 Other Novel Delivery Systems ………………..................................................... 43

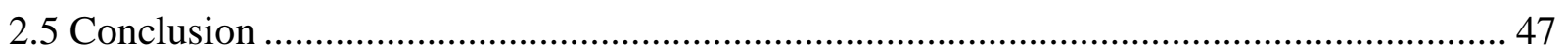

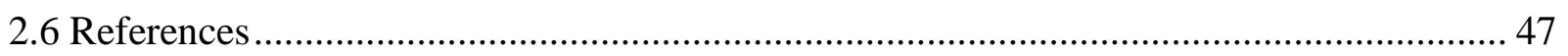

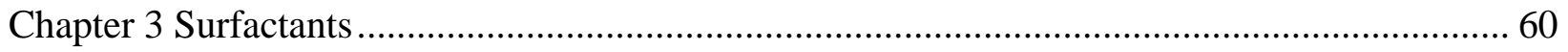

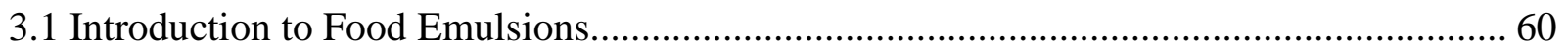

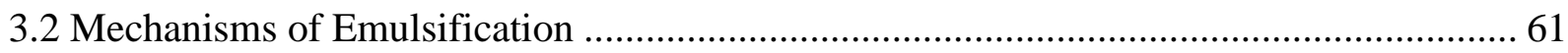

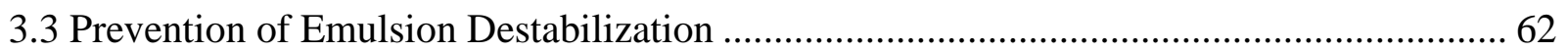

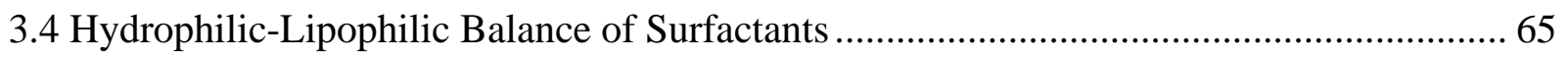

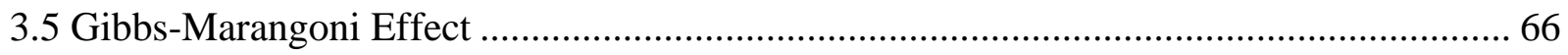

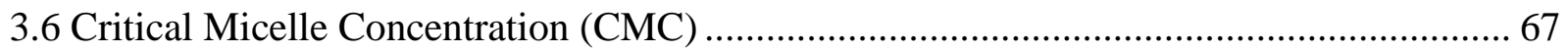

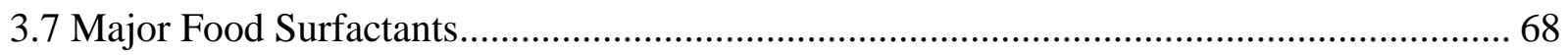

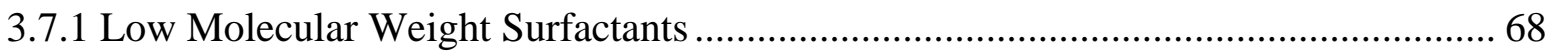




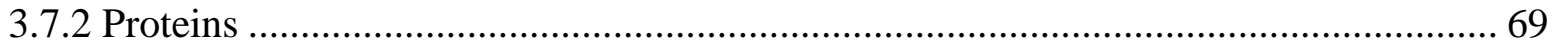

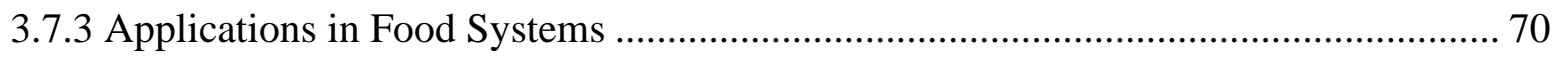

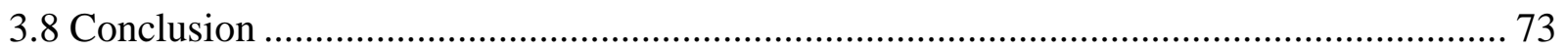

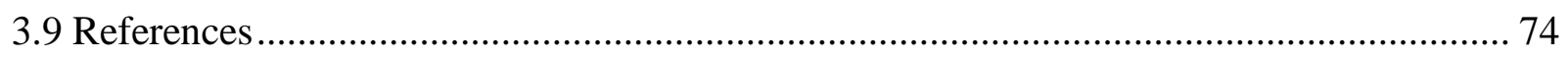

Chapter 4 Lipid Digestion of Oil-in-Water Emulsions Stabilized with Low Molecular Weight

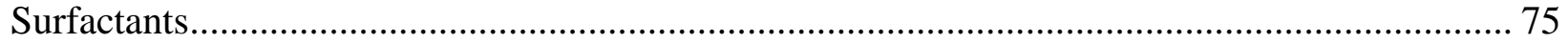

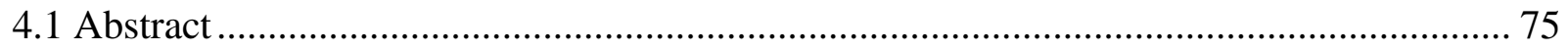

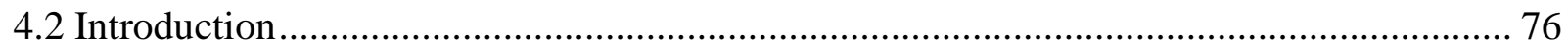

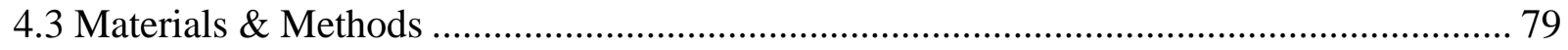

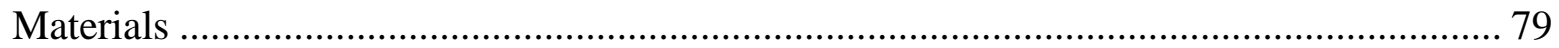

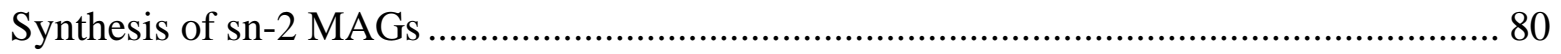

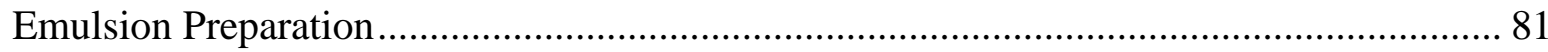

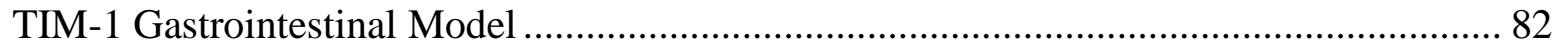

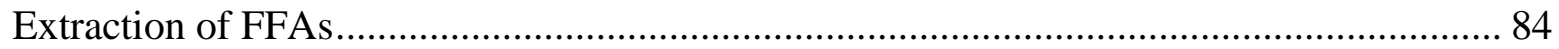

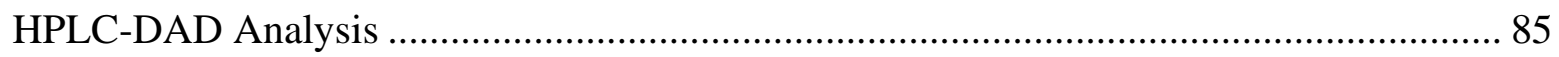

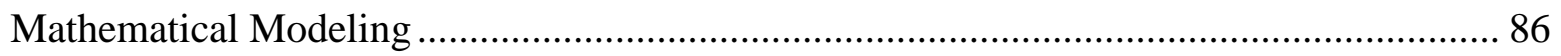

Statistical Analysis .................................................................................................. 87

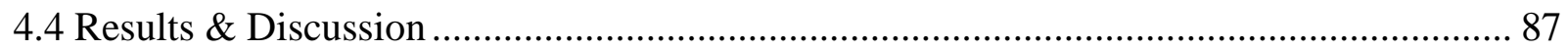

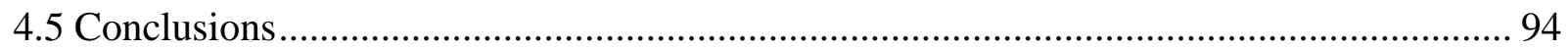

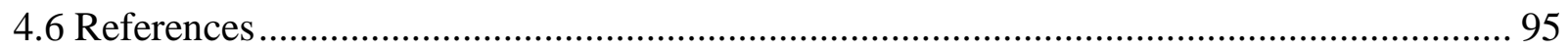

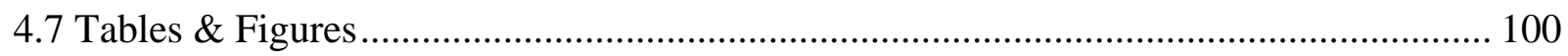

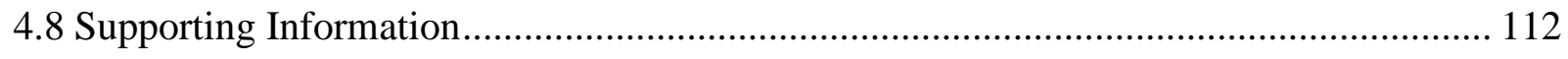

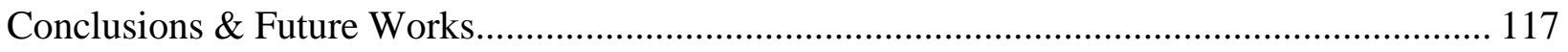




\section{LIST OF TABLES}

Table 4.1 TIM-1 gastric compartment $\mathrm{pH}$ values throughout the $5 \mathrm{~h}$ fed state digestion protocol. 100

Table 4.2 Class, common names, and HLB value of surfactants (Hasenhuettl G, Hartel 2008).101

Table 4.3 Induction time $\left(\mathrm{T}_{\mathrm{c}}\right)$, rate constant $(\mathrm{K})$, and overall bioaccessibility $\left(\mathrm{C}_{\text {asymp }}\right)$ parameters obtained from the shifted logistic model in the jejunum (Jej), ileum (Ile), and combined jejunum

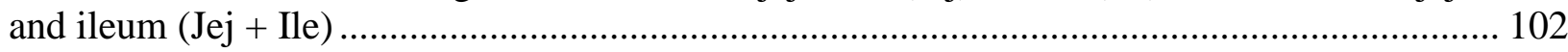




\section{LIST OF FIGURES}

Figure 2.1 Physiological conditions in the gastrointestinal tract (McClements 2013).

Figure 2.2 Destabilization of food emulsions in the acidic environment of the human gastric compartment, beginning with (i) flocculation and leading to various possible structures in the small intestine: (ii) redispersed (stable), (iii) partially-coalesced), (iv) coalesced, or (v) broken, depending on the nature of the emulsion interface and fat composition (Golding et al. 2011).... 14

Figure 2.3 Ileal brake feedback mechanism to induce satiety and slow digestion (Goodman 2012).

Figure 2.4 Overall bioavailability of a bioactive compound encapsulated in a lipid nanoparticle (Joye et al. 2014).

Figure 2.5 Common natural surfactants used to stabilize emulsions (McClements and Gumus 2016)

Figure 2.6 Common phospholipid structures at an emulsion interface (van Nieuwenhuyzen and Szuhaj 2002). 26

Figure 2.7 (a) Deoxycholic acid and (b) schematic representation of facial amphiphilic structure of bile salts (i.e., polar hydroxyl groups on concave side, methyl groups on convex side) (Maldonado-Valderrama et al. 2011). 28

Figure 2.8 Structures of naturally-occurring lipids in food (Meynier and Genot 2017)............. 33

Figure 2.9 Common release mechanisms of micronutrients encapsulated in nanoparticles: (a) diffusion through intact particle matrix, (b) release through particle swelling, (c) particle disintegration, and (d) particle erosion (Joye et al. 2014).

Figure 2.10 Mixed nanoparticle delivery systems with the potential to increase the bioavailability of hydrophobic bioactives in the gastrointestinal tract (GIT) (McClements 2017). 43

Figure 2.11 Various novel delivery systems that have potential to modify lipid digestion (Joye et al. 2014). 46

Figure 3.1 The relationships between different o/w emulsion destabilization mechanisms. Reprinted with permission from Meinders, M. B. J.; Kloek, W.; Van Vliet, T. Effect of Surface Elasticity on Ostwald Ripening in Emulsions. Langmuir 2001, 17, 3923-3929. Copyright 2001 American Chemical Society.

Figure 3.2 Portrayal of the Gibbs-Marangoni effect on two approaching emulsion droplets, with surfactants represented by Y. Reprinted with permission from Walstra, P. Principles of emulsion formation. Chem. Eng. Sci. 1993, 48 (2), 333-349. Copyright 1993 Elsevier..... 67

Figure 3.3 Relationship between interfacial tension, surface hydrophobicity and emulsifying activity of various proteins. Reprinted with permission from Nakai, S. Structure-Function 
Relationships of Food Proteins with an Emphasis on the Importance of Protein Hydrophobicity. J. Agric. Food Chem. 1983, 31 (4), 676-683. Copyright 1983 American Chemical Society...... 70

Figure 3.4 The interfacial components and effect of salts on mayonnaise o/w emulsion. Reprinted with permission from Depree, J. A.; Savage, G. P. Physical and flavour stability of mayonnaise. Trends Food Sci. Technol. 2001, 12 (5-6), 157-163. Copyright 2002 Elsevier

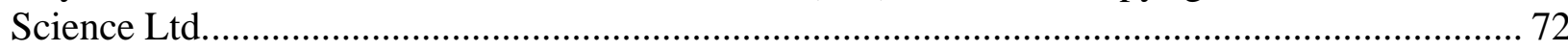

Figure 4.1 Chemical structures of surfactants employed in this study. 103

Figure 4.2 Schematic diagram of the TIM-1 system: A) gastric compartment; B) pyloric sphincter; C) duodenal compartment; D) jejunal compartment; E) ileal compartment; F) pressure sensor; G) gastric secretion; H) duodenal secretion; I) jejunal secretion; J) ileal secretion; K) prefilter; L) semi-permeable membrane (hollow fibre cross-section); M) filtrate pump; N) $\mathrm{pH}$ electrode; O) level sensor; P) temperature sensor; Q) dosing port (Dickinson et al. 2012)...... 104

Figure 4.3 Concentration as a function of average particle size obtained for each surfactantstabilized emulsion measured; $0.5 \%(\mathrm{w} / \mathrm{w})$ was chosen as the lowest concentration at which desired particle size is achieved for all surfactants, except for 2-GMO at $0.7 \%(\mathrm{w} / \mathrm{w})$ concentration. 105

Figure 4.4 Particle size distributions of surfactant-stabilized o/w emulsions stored at $37^{\circ} \mathrm{C}$; emulsions were considered stable when particle size distributions remained monomodal with no major shift in peak over the 7-day period.

Figure 4.5 Absolute caprylic acid bioaccessibility from low molecular weight surfactant stabilized tricaprylin oil-in-water emulsions over a $5 \mathrm{~h}$ simulated digestion period.

Figure 4.6 Cumulative percent bioaccessibility of caprylic acid cleaved from tricaprylin oil-inwater emulsions stabilized with various low molecular weight surfactants over a $5 \mathrm{~h}$ simulated digestion period. 108

Figure 4.7 Induction time obtained from the shifted logistic model of the jejunal filtrate. No significant differences between samples were observed $(p>0.05)$.

Figure 4.8 Rate constant calculated from the shifted logistic model of the jejunal and ileal filtrate. No significant differences between samples were observed $(p>0.05)$.

Figure 4.9 Total caprylic acid bioaccessibility (i.e., $\mathrm{C}_{\text {asymp}}$ ) calculated from the shifted logistic model of the jejunal and ileal filtrate. Different symbols indicate significant differences between samples $(p<0.05)$. 


\section{LIST OF SYMBOLS, ABBREVIATIONS OR NOMENCLATURE}

\begin{tabular}{|c|c|}
\hline GI & Gastrointestinal \\
\hline MAG & Monoacylglyceride \\
\hline DAG & Diacylglyceride \\
\hline TAG & Triacylglyceride \\
\hline FFA & Free fatty acid \\
\hline LMW & Low molecular weight \\
\hline HLB & Hydrophilic-lipophilic balance \\
\hline Span 60 & Sorbitan monostearate \\
\hline Span 80 & Sorbitan monooleate \\
\hline Tween 60 & Polyoxyethylene (20) sorbitan monostearate \\
\hline Tween 80 & Polyoxyethylene (20) sorbitan monooleate \\
\hline GMO & Glycerol monooleate \\
\hline GMS & Glycerol monostearate \\
\hline $\mathrm{T}_{\mathrm{c}}$ & Induction time \\
\hline K & Rate constant \\
\hline $\mathrm{C}_{\text {asymp }}$ & Total bioaccessible fraction of FFAs; asymptotic plateau \\
\hline AUC & Area under the curve \\
\hline
\end{tabular}




\section{Chapter 1 Introduction}

The global diet has become increasingly dominated by energy-dense, ultra-processed foods (UPFs) which are typically high in fat, sugar, and salt (Moubarac et al. 2013; Monteiro et al. 2013). Numerous studies have correlated UPF consumption with increased prevalence of obesity (Louzada et al. 2015; Juul and Hemmingsson 2015; Poti et al. 2017; Monteiro et al. 2018b; Nardocci et al. 2019). This has contributed significantly to the global obesity pandemic and rise in obesity-related non-communicable diseases, including insulin resistance, metabolic syndrome, type 2 diabetes, and coronary heart disease (Misra and Khurana 2011; Ng et al. 2014). Consequently, dietary risk factors have become the leading cause of all-cause mortality, with $\sim 11$ million deaths across 195 countries attributable to dietary risk factors in 2017 (Afshin et al. 2019). Therefore, careful attention to food processing and formulation is necessary to attenuate negative health outcomes associated with diets largely dominated by UPFs (Monteiro et al. 2018a).

Foods with the same nutritional composition and energy content, but of different structure and form, can demonstrate different metabolic responses (Dias et al. 2019). Food matrices can impact the release, digestion, and absorption of lipids (McClements 2018). Therefore, an alternative to reducing fat content in foods, which would negatively impact organoleptic properties (i.e., texture and flavor), is structuring foods to modify lipid digestion and bioavailability (Singh and Ye 2013). Foods are complex systems containing a variety of components, such as dietary fibers, minerals, proteins, carbohydrates, and surfactants, which all have the potential to alter digestion and absorption of lipids (McClements et al. 2009). The body must breakdown food matrices surrounding lipids before lipolysis can occur (Chen et al. 2006). Furthermore, hydrolysis of proteins, polysaccharides, and lipids during digestion produces molecular species capable of 
interfering with lipid digestion and absorption (i.e., amino acids, peptides, fatty acids, monoglycerides) (Vaskonen 2003; Reid 2004; McClements et al. 2009).

Lipid digestion occurs in the oral, gastric, and intestinal segments of the GI tract via action of lipolytic enzymes (Guo et al. 2017). Lipolysis is initiated in the stomach by acid-stable lingual and gastric lipases (Moreau et al. 1988; Wahbeh and Christie 2011). In adults, lingual lipase secreted by the serous Von Ebner glands of the tongue do not significantly contribute to overall lipolysis; lingual lipase is more significant in infants or patients with severe pancreatic insufficiency (Abrams et al. 1987; Hamosh 1990; Mu and Høy 2004). Gastric lipases cleave $s n-3$ ester bonds of triacylglycerides (TAGs), resulting in $s n-1,2$ diacylglycerides (DAGs) and free fatty acids (FFAs); these gastric lipolytic products act as emulsifying agents to facilitate digestion in the small intestine where lipid absorption primarily occurs (Ramírez et al. 2001; Singh and Ye 2013; Guo et al. 2017). Gastric lipolytic activity hydrolyzes $~ 10-30 \%$ of ingested TAGs in human adults (Singh and Ye 2013). Pancreatic lipase is responsible for $40-70 \%$ of TAG lipolysis and cleaves both $s n-1$ and $s n-3$ ester bonds to release two FFAs and $s n-2$ monoacylglycerides (Wilde and Chu 2011; Meynier and Genot 2017). Pancreatic lipase requires co-lipase to reduce interfacial tension facilitating adsorption of lipase at oil-water interface in the presence of bile salts (Singh and Ye 2013). Lipid absorption occurs in two ways: (1) passive diffusion through the brush border membrane of enterocytes, and (2) transport to brush border membrane via transporters (i.e., fatty acid transport protein 4, FATP4) (Meynier and Genot 2017).

Different approaches to controlling the rate and extent of lipid digestion of food emulsions, include controlling the characteristics of the lipid phase, lipid droplet size, and interfacial properties of lipid droplets (McClements et al. 2009). For instance, long chain saturated fatty acids are poorly absorbed compared to short or medium chain fatty acids due to their tendency to form 
insoluble soaps with multivalent ions (Karupaiah and Sundram 2007; Thilakarathna et al. 2016). The oil droplet size of emulsions determines the interfacial surface area available for enzyme activity (Verkempinck et al. 2018). Smaller droplet sizes $(<1 \mu \mathrm{m})$ correspond to greater interfacial surface area available for lipase adsorption, thus faster lipolysis rates and higher lipid digestibility. Conversely, larger droplet sizes $(>10 \mu \mathrm{m})$ result in less surface area and confer a slower lipolysis rate (Salvia-Trujillo et al. 2013, 2017; Zhang and McClements 2016; Guo et al. 2017). Interfacial composition may affect the stability of lipid droplets to coalescence in the GI tract and the ability of lipases (also co-lipases, phospholipids, and bile salts) to adsorb to the interface and act upon TAG substrates (Reis et al. 2006; Sonesson et al. 2007). Surfactants at the oil-water interface may inhibit lipase via steric hindrance, interfacial complex formation, direct interaction with lipase, and interaction with other digestive compounds (i.e., bile) (Favé et al. 2004; Mun et al. 2007; Golding and Wooster 2010; Speranza et al. 2013).

By influencing lipid bioavailability, food structure also has the potential to modulate other physiological responses such as satiety and post-prandial lipemia (Gallier et al. 2014; Dias et al. 2019). The lipemic index is similar to the well-established glycemic index and represents postprandial serum triacylglyceride (TAG) levels following ingestion of a meal relative to a reference meal, which contains a fixed load (i.e., 50-70 g) of a mixture of saturated, polyunsaturated and monounsaturated fats in known quantities (Ooi et al. 2011). Elevated post-prandial lipemia is a criterion for metabolic syndrome and recognized as a risk factor for obesity-related chronic diseases, such as type-2 diabetes and cardiovascular diseases (Ardern and Janssen 2007; Dekker et al. 2009). Food structure plays an important role in modulating post-prandial lipemia by influencing the rate and extent of lipolysis (Dias et al. 2017, 2019). For example, intake of food emulsions stabilized by different surfactants (i.e., egg lecithin, sodium stearoyl lactylate, and 
sodium caseinate/monoglyceride) have been shown to result in different levels of post-prandial plasma TAG concentrations; thus demonstrating that food can be structured to reduce postprandial TAG levels without changing the amount of food intake (Keogh et al. 2011).

Monoglycerides are glycerol esters of fatty acids, most commonly-used in the food industry for bakery products (i.e., bread, cake, cake mixes) and frozen desserts, and are classified as nonionic oil-soluble surfactants (Weiss 1971; Chen 2015). Monoglycerides have two isomeric forms (i.e., $s n-1$ and $s n$-2) based on a temperature-dependent equilibrium (Lauridsen 1976). Both isomeric forms revert to an equilibrium mixture of predominantly the $s n$-1 isomer (>90\%) (Hudson 2004). Commercial monoglycerides typically contain $95 \% s n-1 / 3$ isomers and $5 \% s n$-2 isomers (Krog and Sparso 2004). Therefore, commercial-grade monoglycerides and common non-ionic synthetic surfactants (i.e., Spans and Tweens) are expected to be more effective surfactants and better at reducing particle size compared to predominantly $s n-2$ isomers of MAGs, due to symmetry of the attached hydroxyl groups for sn-2 MAG molecules (Lauridsen 1976; Krog 1977).

As surfactants are widely used in the food industry to increase emulsion stability, it is important to consider the physicochemical properties of surfactants on their physiological impact (Speranza et al. 2013; McClements 2018). While the food industry is not responsible for managing consumer lifestyle choices, food manufacturers should strive to create food products with a greater focus on their healthfulness (Lundin and Golding 2009). Overall, understanding the relationship between food properties, digestion, and absorption will facilitate the design of foods with improved health outcomes (Sanguansri and Augustin 2006; Parada and Aguilera 2007). 


\subsection{Objectives}

The objective of this study was to investigate the effects of $s n$-position for monoglycerides and the structure of non-ionic, synthetic surfactants on the rate and extent of lipid digestion of oilin-water (o/w) emulsions using the in vitro TIM-1 simulated digestion model. This was achieved by comparing the lipid digestion profiles of oil-in-water $(\mathrm{o} / \mathrm{w})$ emulsions stabilized with one of the following surfactants:
a) Span 60 (sorbitan monostearate)
b) Span 80 (sorbitan monooleate)
c) Tween 60 (polyoxyethylene (20) sorbitan monostearate)
d) Tween 80 (polyoxyethylene (20) sorbitan monooleate)
e) Commercial glycerol monostearate (1-GMS)
f) Commercial glycerol monooleate (1-GMO)
g) $S n$-2 glycerol monooleate (2-GMO)

\subsection{Hypothesis}

Sn-2 monoglycerides will reduce the rate and extent of lipid digestion by forming a monolayer around emulsion droplets that is more difficult to displace by bile compared to commercial monoglycerides and non-ionic, synthetic surfactants. 


\subsection{References}

Abrams CK, Hamosh M, Dutta SK, et al (1987) Role of nonpancreatic lipolytic activity in exocrine pancreatic insufficiency. Gastroenterology 92:125-129 . doi: 10.1016/00165085(87)90848-1

Afshin A, Sur PJ, Fay KA, et al (2019) Health effects of dietary risks in 195 countries, 19902017: a systematic analysis for the Global Burden of Disease Study 2017. Lancet. doi: 10.1016/S0140-6736(19)30041-8

Ardern CI, Janssen I (2007) Metabolic syndrome and its association with morbidity and mortality. Appl Physiol Nutr Metab 32:33-45 . doi: 10.1139/h06-099

Chen L (2015) Emulsifiers as food texture modifiers. In: Modifying Food Texture: Novel Ingredients and Processing Techniques. pp 27-49

Chen L, Remondetto GE, Subirade M (2006) Food protein-based materials as nutraceutical delivery systems. Trends Food Sci Technol 17:272-283 . doi: 10.1016/j.tifs.2005.12.011

Dekker MJ, Wright AJ, Mazurak VC, et al (2009) Fasting triacylglycerol status, but not polyunsaturated/saturated fatty acid ratio, influences the postprandial response to a series of oral fat tolerance tests. J Nutr Biochem 20:694-704 . doi: 10.1016/j.jnutbio.2008.06.012

Dias CB, Moughan PJ, Wood LG, et al (2017) Postprandial lipemia: factoring in lipemic response for ranking foods for their healthiness. Lipids Health Dis 16:178 . doi: 10.1186/s12944-017-0568-5

Dias CB, Zhu X, Thompson AK, et al (2019) Effect of the food form and structure on lipid digestion and postprandial lipaemic response. Food Funct 10:112-124 . doi: $10.1039 / \mathrm{c} 8$ fo0 $1698 \mathrm{~d}$

Favé G, Coste TC, Armand M (2004) Physicochemical properties of lipids: new strategies to manage fatty acid bioavailability. Cell Mol Biol (Noisy-le-grand) 50:815-31

Gallier S, Rutherfurd SM, Moughan PJ, Singh H (2014) Effect of food matrix microstructure on stomach emptying rate and apparent ileal fatty acid digestibility of almond lipids. Food Funct 5:2410-2419 . doi: 10.1039/c4fo00335g

Golding M, Wooster TJ (2010) The influence of emulsion structure and stability on lipid digestion. Curr Opin Colloid Interface Sci 15:90-101 . doi: 10.1016/j.cocis.2009.11.006

Guo Q, Ye A, Bellissimo N, et al (2017) Modulating fat digestion through food structure design. Prog Lipid Res 68:109-118 . doi: 10.1016/j.plipres.2017.10.001

Hamosh M (1990) Lingual and gastric lipases. Nutrition 6:421-8

Hudson BJF (2004) Fatty Acids | Properties. In: Encyclopedia of Food Sciences and Nutrition. Academic Press, pp 2297-2300

Juul F, Hemmingsson E (2015) Trends in consumption of ultra-processed foods and obesity in Sweden between 1960 and 2010. Public Health Nutr 18:3096-3107 . doi: $10.1017 /$ S1368980015000506

Karupaiah T, Sundram K (2007) Effects of stereospecific positioning of fatty acids in triacylglycerol structures in native and randomized fats: A review of their nutritional implications. Nutr Metab 4:16 . doi: 10.1186/1743-7075-4-16

Keogh JB, Wooster TJ, Golding M, et al (2011) Slowly and Rapidly Digested Fat Emulsions Are Equally Satiating but Their Triglycerides Are Differentially Absorbed and Metabolized in Humans. J Nutr 141:809-815 . doi: 10.3945/jn.110.131110

Krog N (1977) Functions of emulsifiers in food systems. J Am Oil Chem Soc 54:124-131 . doi: 10.1007/BF02894388 
Krog NJ, Sparso FV (2004) Food emulsifiers: their chemical and physical properties. In: Food Emulsions, 4th ed. pp 45-91

Lauridsen JB (1976) Food emulsifiers: Surface activity, edibility, manufacture, composition, and application. J Am Oil Chem Soc 53:400-407 . doi: 10.1007/BF02605731

Louzada ML da C, Baraldi LG, Steele EM, et al (2015) Consumption of ultra-processed foods and obesity in Brazilian adolescents and adults. Prev Med (Baltim) 81:9-15 . doi:

10.1016/J.YPMED.2015.07.018

Lundin L, Golding M (2009) Structure design for healthy food. Aust J Dairy Technol 64:68-75

McClements DJ (2018) The biophysics of digestion: lipids. Curr Opin Food Sci 21:1-6 . doi: 10.1016/j.cofs.2018.03.009

McClements DJ, Decker EA, Park Y (2009) Controlling lipid bioavailability through physicochemical and structural approaches. Crit Rev Food Sci Nutr 49:48-67 . doi: 10.1080/10408390701764245

Meynier A, Genot C (2017) Molecular and structural organization of lipids in foods: their fate during digestion and impact in nutrition. OCL 24:D202 . doi: 10.1051/ocl/2017006

Misra A, Khurana L (2011) Obesity-related non-communicable diseases: South Asians vs White Caucasians. Int J Obes 35:167-187 . doi: 10.1038/ijo.2010.135

Monteiro CA, Cannon G, Moubarac J-C, et al (2018a) The UN Decade of Nutrition, the NOVA food classification and the trouble with ultra-processing. Public Health Nutr 21:5-17 . doi: $10.1017 /$ S1368980017000234

Monteiro CA, Moubarac J-C, Cannon G, et al (2013) Ultra-processed products are becoming dominant in the global food system. Obes Rev 14:21-28 . doi: 10.1111/obr.12107

Monteiro CA, Moubarac J-C, Levy RB, et al (2018b) Household availability of ultra-processed foods and obesity in nineteen European countries. Public Health Nutr 21:18-26 . doi: $10.1017 /$ S1368980017001379

Moreau H, Laugier R, Gargouri Y, et al (1988) Human preduodenal lipase is entirely of gastric fundic origin. Gastroenterology 95:1221-1226 . doi: 10.1016/0016-5085(88)90354-X

Moubarac J-C, Martins APB, Claro RM, et al (2013) Consumption of ultra-processed foods and likely impact on human health. Evidence from Canada. Public Health Nutr 16:2240-8 . doi: $10.1017 / \mathrm{S} 1368980012005009$

Mu H, Høy CE (2004) The digestion of dietary triacylglycerols. Prog Lipid Res 43:105-133 . doi: 10.1016/S0163-7827(03)00050-X

Mun S, Decker EA, McClements DJ (2007) Influence of emulsifier type on in vitro digestibility of lipid droplets by pancreatic lipase. Food Res Int 40:770-781 . doi: 10.1016/j.foodres.2007.01.007

Nardocci M, Leclerc B-S, Louzada M-L, et al (2019) Consumption of ultra-processed foods and obesity in Canada. Can J Public Heal 110:4-14 . doi: 10.17269/s41997-018-0130-x

$\mathrm{Ng} \mathrm{M}$, Fleming T, Robinson M, et al (2014) Global, regional, and national prevalence of overweight and obesity in children and adults during 1980-2013: A systematic analysis for the Global Burden of Disease Study 2013. Lancet 384:766-781 . doi: 10.1016/S01406736(14)60460-8

Ooi TC, Robinson L, Graham T, et al (2011) Proposing a "lipemic index" as a nutritional and research tool. Curr Vasc Pharmacol 9:313-7

Parada J, Aguilera JM (2007) Food microstructure affects the bioavailability of several nutrients. J Food Sci 72:R21-R32 . doi: 10.1111/j.1750-3841.2007.00274.x

Poti JM, Braga B, Qin B (2017) Ultra-processed Food Intake and Obesity: What Really Matters 
for Health-Processing or Nutrient Content? Curr Obes Rep 6:420-431 . doi: 10.1007/s13679-017-0285-4

Ramírez M, Amate L, Gil A (2001) Absorption and distribution of dietary fatty acids from different sources. Early Hum Dev 65:S95-S101 . doi: 10.1016/S0378-3782(01)00211-0

Reid IR (2004) Effects of Calcium Supplementation on Circulating Lipids: Potential Pharmacoeconomic Implications. Drugs and Aging 21:7-17 . doi: 10.2165/00002512200421010-00002

Reis P, Holmberg K, Debeche T, et al (2006) Lipase-catalyzed reactions at different surfaces. Langmuir 22:8169-8177 . doi: 10.1021/la060913s

Salvia-Trujillo L, Qian C, Martín-Belloso O, McClements DJ (2013) Influence of particle size on lipid digestion and $\beta$-carotene bioaccessibility in emulsions and nanoemulsions. Food Chem 141:1475-1480 . doi: 10.1016/j.foodchem.2013.03.050

Salvia-Trujillo L, Verkempinck SHE, Sun L, et al (2017) Lipid digestion, micelle formation and carotenoid bioaccessibility kinetics: Influence of emulsion droplet size. Food Chem 229:653-662 . doi: 10.1016/j.foodchem.2017.02.146

Sanguansri P, Augustin MA (2006) Nanoscale materials development - a food industry perspective. Trends Food Sci Technol 17:547-556 . doi: 10.1016/j.tifs.2006.04.010

Singh H, Ye A (2013) Structural and biochemical factors affecting the digestion of proteinstabilized emulsions. Curr Opin Colloid Interface Sci 18:360-370 . doi: 10.1016/j.cocis.2013.04.006

Sonesson AW, Brismar H, Callisen TH, Elofsson UM (2007) Mobility of Thermomyces lanuginosus lipase on a trimyristin substrate surface. Langmuir 23:2706-2713 . doi: $10.1021 / \mathrm{la} 062003 \mathrm{~g}$

Speranza A, Corradini MG, Hartman TG, et al (2013) Influence of emulsifier structure on lipid bioaccessibility in oil-water nanoemulsions. J Agric Food Chem 61:6505-6515 . doi: $10.1021 / \mathrm{jf} 401548 \mathrm{r}$

Thilakarathna SH, Rogers M, Lan Y, et al (2016) Investigations of in vitro bioaccessibility from interesterified stearic and oleic acid-rich blends. Food Funct 7:1932-1940 . doi: $10.1039 / \mathrm{c} 5$ fo0 $01272 \mathrm{~d}$

Vaskonen T (2003) Dietary minerals and modification of cardiovascular risk factors. J Nutr Biochem 14:492-506 . doi: 10.1016/S0955-2863(03)00074-3

Verkempinck SHE, Salvia-Trujillo L, Moens LG, et al (2018) Emulsion stability during gastrointestinal conditions effects lipid digestion kinetics. Food Chem 246:179-191 . doi: 10.1016/j.foodchem.2017.11.001

Wahbeh GT, Christie DL (2011) Basic Aspects of Digestion and Absorption. In: Pediatric Gastrointestinal and Liver Disease. W.B. Saunders, pp 10-19.e2

Weiss TJ (1971) Food emulsifiers-Science and art. J Am Oil Chem Soc 49:457-461

Wilde PJ, Chu BS (2011) Interfacial \& colloidal aspects of lipid digestion. Adv Colloid Interface Sci 165:14-22 . doi: 10.1016/j.cis.2011.02.004

Zhang R, McClements DJ (2016) Enhancing nutraceutical bioavailability by controlling the composition and structure of gastrointestinal contents: Emulsion-based delivery and excipient systems. Food Struct 10:21-36 . doi: 10.1016/j.foostr.2016.07.006 


\section{Chapter 2 Literature Review}

\subsection{Introduction}

With rising prevalence of diet-related diseases, careful consideration must be given to the processing and formulation of foods (Speranza et al. 2013). The interaction between food and the human body is extremely complex and influenced by food composition, properties, structure, time of consumption, and previous foods consumed (Sensoy 2014). Food structure, not just composition, influences digestion and metabolism (Golding and Wooster 2010). Food structure can be manipulated to influence the rate of release and absorption of metabolites in the gastrointestinal (GI) tract (Sensoy 2014; McClements 2018). Food matrices can alter the rheology of GI fluids to slow diffusion and mixing, act as physical barriers to limit access of digestive enzymes, and/or sequester digestion components (i.e., bile salts, enzymes) (McClements 2018).

Fat digestion significantly influences health, as elevated blood serum fatty acid concentrations leads to insulin resistance, inflammation, and increased risk of metabolic syndrome (Boden 2008). Lipolysis converts water-insoluble lipids into amphiphilic fatty acids and $s n-2$ monoacylglycerides (2-MAGs) that are incorporated into water-soluble bile salt/phospholipid micelles and vesicles that are transported to enterocyte cells for absorption (Stipanuk 2006; Hur et al. 2009). Dietary lipids are typically emulsified into droplets within foods or during digestion in the GI tract; emulsification increases surface area allowing bile, co-lipase and lipase to access the interface facilitating lipolysis (Wilde and Chu 2011). The majority of fatty acid and 2-MAG absorption occurs in the duodenum and jejunum, but in cases of excess consumption or malabsorption, undigested foods travel to the ileum. This triggers hormonal secretions that slow digestion and signals the brain to reduce appetite. The ileal brake is a negative feedback mechanism whereby the presence of ingested food triggers hormonal signals that inhibit proximal GI motility 
and gastric emptying (Cummings et al. 2007). Modifying the ileal brake mechanism modulates hunger, satiety and satiation, and may aid in weight management (Maljaars et al. 2008a).

An alternative to reducing fat content in foods, which is deleterious to organoleptic properties (i.e., texture and flavour), is structuring lipids to have reduced digestibility and bioavailability (generally referred to as the lipemic index) (Singh and Ye 2013). Foods may be structured to decrease lipid bioavailability to target individuals with high blood lipid levels at risk for cardiovascular disease or obesity. Conversely, foods may be structured to increase lipid bioavailability to target individuals with diseases that affect digestion and absorption of lipids (i.e. pancreatitis, cystic fibrosis) (Mun et al. 2007; Singh et al. 2009). To effectively structure foods to modify lipid bioavailability, it is important to correlate physicochemical properties of foods to physiological events in the body (McClements 2018). Interfacial access in complex foods requires additional physicochemical changes to occur during digestion to breakdown these matrices. Important interfacial characteristics include: interfacial area, competitive adsorption of emulsifiers, and other surface-active compounds (McClements 2018).

\subsection{Lipid Digestion}

Lipid digestion hydrolyzes triacylglycerides (TAGs) into fatty acids and 2-MAGs that are either absorbed via passive or facilitated diffusion (Guo et al. 2017). Lipids are not only an important energy source, but also carry essential lipid-soluble vitamins (i.e., A, D, E, and K), carotenoids, flavonoids, and some drugs (Wilde and Chu 2011; Meynier and Genot 2017). Lipid digestion occurs in oral, gastric, and intestinal segments of the GI tract (Figure 2.1) (Guo et al. 2017). 


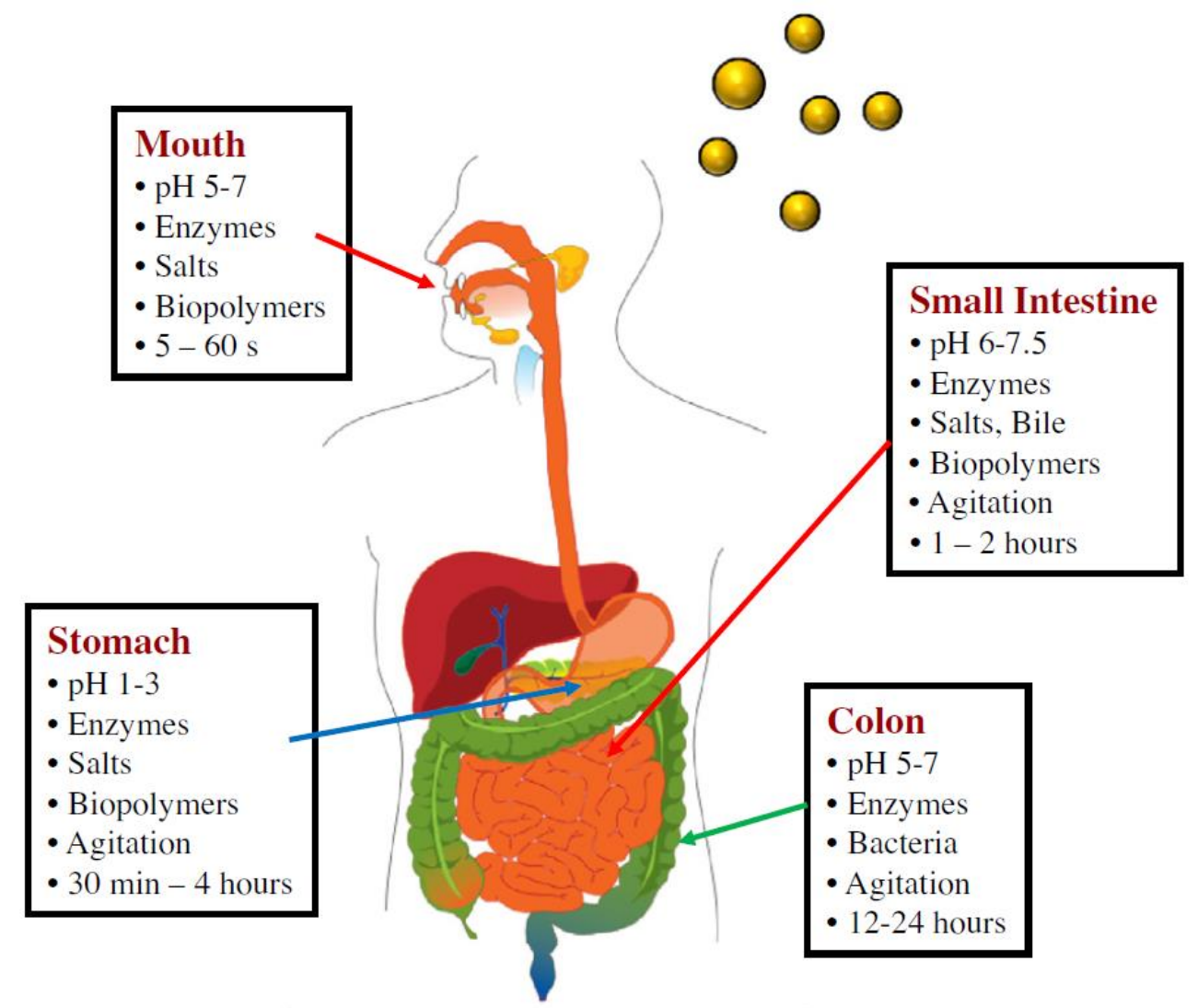

Figure 2.1 Physiological conditions in the gastrointestinal tract (McClements 2013).

\subsubsection{Oral Phase}

Lipid digestion begins with mechanical and biochemical modifications in the oral cavity (Singh and Ye 2013). Solid and semi-solid foods are masticated, releasing oil droplets, and mixed with saliva to form a bolus before swallowing (Singh and Ye 2013; Guo et al. 2017; Meynier and Genot 2017). The mechanical shear experienced by food between teeth, tongue, and oral palate reduces particle size, increases surface area, and aids nutrient release (McClements 2018). Liquid emulsions are mixed with saliva and swallowed immediately. Saliva ( $\mathrm{pH}$ of $\sim 6.8$ ) consists of water, proteins (i.e., lingual lipase, $\alpha$-amylase, and negatively-charged mucins) and minerals (Vingerhoeds et al. 2005; Singh and Ye 2013). In human adults, a limited amount of lipolysis occurs due to lingual lipases secreted by the serous Von Ebner glands of the tongue; lingual lipase is more important in human infants or patients with severe pancreatic insufficiency (Abrams et al. 
1987; Hamosh 1990a; Mu and Høy 2004). Alpha-amylase hydrolyzes starch to oligosaccharides and maltose (McClements 2013). Mineral ions (i.e., $\mathrm{Ca}^{2+}, \mathrm{K}^{+}$) facilitate droplet aggregation in emulsions via electrostatic screening or ion-binding effects (Keowmaneechai and McClements 2006; Chang and McClements 2016). Mucin interacts with positively-charged protein-stabilized emulsions (i.e., lactoferrin) and induces flocculation via electrostatic interactions (Singh and Ye 2013). In the oral cavity, foods are subject to changes in $\mathrm{pH}$, ionic strength, temperature, complex flow/force patterns, and interactions with oral cavity surfaces (McClements 2013). The oral phase is often omitted in in vitro digestion studies; however, these changes have been shown to influence subsequent digestion (Singh and Ye 2013).

\subsubsection{Gastric Phase}

After swallowing, the bolus travels down the esophagus into the gastric cavity (pH 1-2), where gastric secretions and mechanical forces from peristalsis further degrade food structure (McClements 2018). Proteolytic pepsin has optimal hydrolytic activity at $\mathrm{pH} 2$ and at temperatures ranging between 37 and $42^{\circ} \mathrm{C}$ (Singh and Ye 2013). Pepsin preferentially cleaves peptide bonds of aromatic and hydrophobic amino acids that typically reside in the interior of proteins before denaturation. Denaturation is triggered in the stomach by the drastic $\mathrm{pH}$ reduction (Singh and $\mathrm{Ye}$ 2013). Lipolysis in the stomach is catalyzed by acid-stable lingual and gastric lipases (Moreau et al. 1988; Wahbeh and Christie 2011). Gastric lipase, secreted by chief cells in the gastric fundic mucosa, remains active over a wide pH range (i.e., pH 3-6) (Meynier and Genot 2017). Gastric lipase is largely responsible for pre-duodenal lipolytic activity $(\sim 118 \mathrm{U} / \mathrm{g} ; 1 \mathrm{U}=1 \mu \mathrm{mol}$ oleic acid released/min from hydrolysis of trioleylglycerol), while lingual lipase is only present in trace amounts ( 0.3 U/g) (DeNigris et al. 1988). Gastric lipase cleaves $s n-3$ ester bonds of TAGs, resulting in sn-1,2 diacylglycerides (DAGs) and free fatty acids (FFAs) (Singh and Ye 2013). 
Gastric lipolytic products act as emulsifying agents facilitating digestion in the small intestine where lipid absorption primarily occurs (Ramírez et al. 2001; Guo et al. 2017). However, short and medium chain FFAs $(<\mathrm{C} 12)$ can also be absorbed via passive diffusion through the gastric mucosa and enter the portal vein prior to intestinal absorption; thus, medium chain TAGs have been used to reduce fat malabsorption and increase caloric intake in the management of certain GI disorders (i.e., pancreatic or bile insufficiency) (Bracco 1994; Parrish 2017).

Surface-active materials in the stomach may originate from foods (i.e., proteins, polysaccharides, phospholipids, surfactants), lipolytic products (i.e., 1,2-DAGs, FFAs) or gastric secretions (i.e., enzymes, glyco-proteins, phospholipids) (Pafumi et al. 2002; Kalantzi et al. 2006). Bile salt solubilization of surface-active molecules begins in the duodenum; thus, surface-active molecules in the stomach may accumulate at the interface and impede lipase accessibility to the substrate (Reis et al. 2008). Overall, gastric lipolytic activity hydrolyzes $~ 10-30 \%$ of ingested TAGs in human adults (Singh and Ye 2013). The main purpose of gastric lipase is to facilitate subsequent pancreatic lipase activity by promoting lipid emulsification and generation of fatty acids and diglycerides (Hamosh et al. 1975; Hamosh 1990b; Carriere et al. 1993; Armand et al. 1994, 1996). Transit time of food in the gastric phase can range from minutes to hours depending on its quantity, physical state, structure, and location in the stomach, resulting in: matrix breakdown, lipid droplet coalescence, and changes in interfacial composition (Figure 2.2) (McClements et al. 2008). 


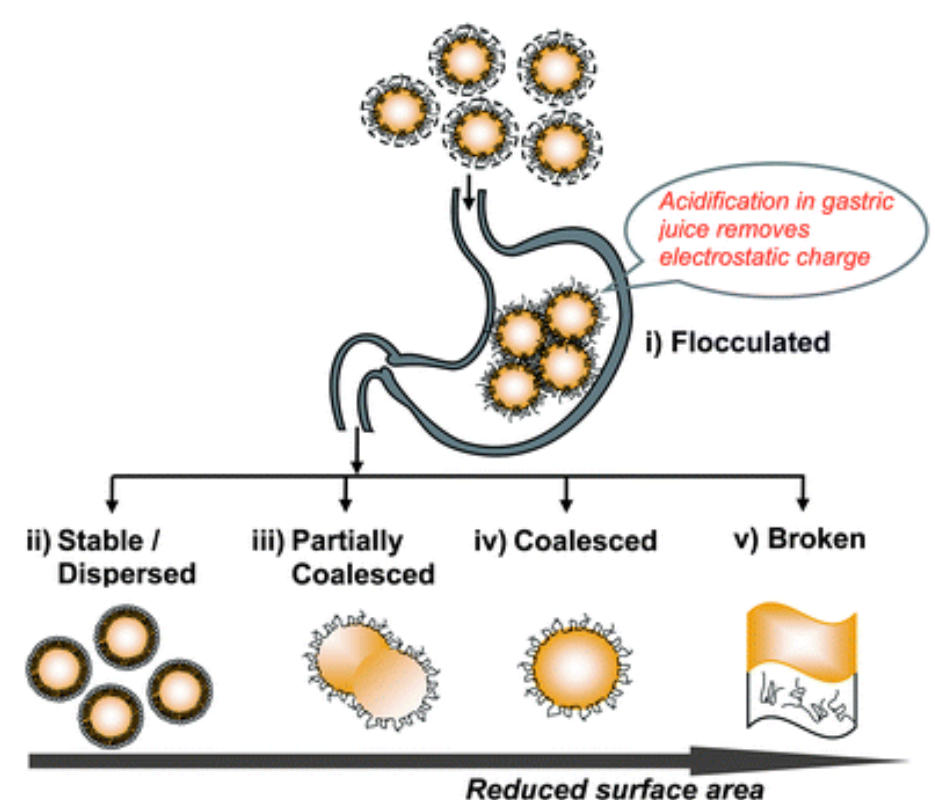

Figure 2.2 Destabilization of food emulsions in the acidic environment of the human gastric compartment, beginning with (i) flocculation and leading to various possible structures in the small intestine: (ii) redispersed (stable), (iii) partially-coalesced), (iv) coalesced, or (v) broken, depending on the nature of the emulsion interface and fat composition (Golding et al. 2011).

\subsubsection{Intestinal Phase}

Partially-digested food, now known as chyme, travels through the pylorus sphincter into the small intestine. This is the main site for lipid digestion where food is broken down into smaller sub-units for absorption at the epithelial lining (Singh and Ye 2013; McClements and Gumus 2016). The transit of food into the small intestine triggers gall bladder secretions of digestive enzymes and bile salts (Singh and Sarkar 2011). Chyme is mixed with intestinal fluids containing bicarbonate, proteases, pancreatic lipase, bile salts, phospholipids, co-lipase, and other salts. The addition of sodium bicarbonate neutralizes $\mathrm{pH}$ allowing maximum pancreatic enzyme activity (Singh and Ye 2013; McClements 2013). Enterokinase is synthesized by the enterocytes of the small intestine in order to activate trypsinogen, the zymogen of trypsin. Active trypsin, generated via enterokinase or autoactivation, subsequently triggers an activation cascade of the remaining pancreatic zymogens (i.e., chymotrypsinogen, proelastase, kallikreinogen, etc.) (Rinderknecht 
2005). The main proteases present in the intestinal phase are trypsin and chymotrypsin, each with different substrate specificities (Ma et al. 2005; Singh and Ye 2013). Trypsin preferentially cleaves the peptide bonds to the C-terminal side of amino acids (i.e., lysine and arginine); while, chymotrypsin favours peptide bonds neighboring large aromatic residues (i.e., phenylalanine, tyrosine, and tryptophan) (Vajda and Szabo 1976; Olsen et al. 2004; Ma et al. 2005).

Pancreatic lipase is synthesized by the acinar cells of the pancreas and has an optimum $\mathrm{pH}$ of 8-9 (Singh and Ye 2013). It is responsible for $\sim 40-70 \%$ of TAG lipolysis and cleaves both $s n-1$ and $s n-3$ ester bonds to release FFAs and 2-MAGs (Wilde and Chu 2011; Meynier and Genot 2017). Solubilization of lipids and lipid-soluble nutrients rely on the adsorption of lipase and emulsification of FFAs and 2-MAGs at the oil-water interface (Patton and Carey 1979). Pancreatic lipase requires co-lipase to reduce interfacial tension facilitating adsorption of lipase at oil-water interface in the presence of bile salts. Thus, pancreatic lipase activity depends on competitive adsorption between bile salts and surface-active materials, lipase/co-lipase adsorption, and removal of lipolytic products by bile salts (Wilde and Chu 2011; Singh and Ye 2013). The most surface-active lipolysis product is 2-MAGs, followed by FFAs, DAGs, and TAGs (Golding and Wooster 2010).

Bile, produced in the liver and stored in the gall bladder, is secreted into the duodenum in response to the presence of food. It is composed of protonated bile acids and deprotonated bile salts (Wilde and Chu 2011). Bile is responsible for the continual displacement of surface-active molecules at the oil-water interface after the initiation of lipolysis (Reis et al. 2008). Due to its high surface activity, bile salts limit pancreatic lipase access to the oil droplet interface. Hence, lipase requires a co-factor (co-lipase) that synergistically reduces interfacial tension and allows adsorption of lipase onto the oil droplet interface in the presence of bile salts (Wilde and Chu 
2011). Lipolytic products then must be incorporated into mixed micelles $(8-20 \mathrm{~nm})$ containing bile salts or larger vesicles (40-200 nm) containing phospholipids to be absorbed (Meynier and Genot 2017). Mixed micelles and vesicles act as vehicles for solubilized FFAs, MAGs, oil-soluble nutraceuticals and vitamins (McClements 2013). Lipid absorption occurs in two ways: (1) passive diffusion through the brush border membrane of enterocytes, and (2) transport to brush border membrane via transporters - i.e., FATP4 (fatty acid transport protein 4), CD36 or SR-B1 (scavenger receptor class B1 for cholesterol) (Meynier and Genot 2017).

The presence of nutrients (especially lipids and proteins) in the duodenum and jejunum triggers the release of cholecystokinin (CCK), a peptide that induces satiety and reduces food intake (Lieverse et al. 1995). Of greater significance is the "ileal brake", a distal to proximal negative feedback mechanism that controls transit time through the GI tract to maximize nutrient digestion and absorption by inhibiting proximal GI motility and secretions (Maljaars et al. 2008a). Two peptides have been demonstrated to be key mediators of the ileal brake: peptide YY (PYY) and glucagon-like peptide 1 (GLP-1) (Schirra and Göke 2005; Stanghellini et al. 2016). Both peptides are secreted by L-cells in the ileum and colon in response to fatty acids and carbohydrates, resulting in inhibition of gastric and duodenal motility. Overall, the activation of the ileal brake enhances gastric distension from delayed emptying, stimulates central satiety centers by hormonal and neural signals, increases satiety, and reduces energy intake (Figure 2.3) (Maljaars et al. 2008a). 


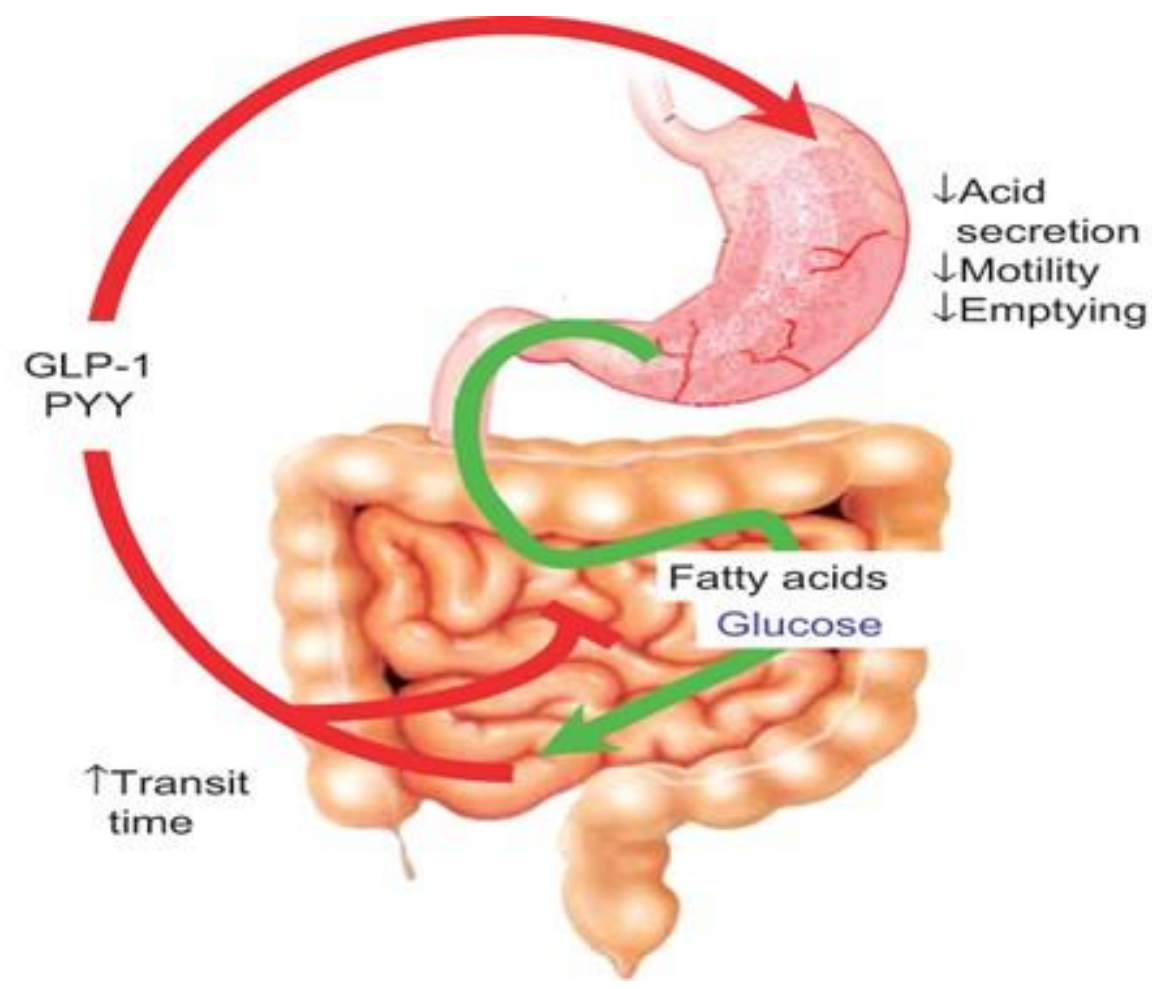

Figure 2.3 Ileal brake feedback mechanism to induce satiety and slow digestion (Goodman 2012).

\subsubsection{Bioaccessibility \& Bioavailability}

Bioaccessibility is defined as the fraction of an ingested compound solubilized within intestinal fluids and available for absorption (Salvia-Trujillo et al. 2013a). On the other hand, bioavailability represents the fraction of ingested compound that reaches systemic circulation in its active form and measures bioactive functionality, not just serum concentration (Yao et al. 2015). There are two major motivations for controlling lipid bioavailability: (1) to increase lipid bioavailability of beneficial lipids and lipid-soluble compounds for those who suffer from absorption insufficiencies; and (2) to decrease lipid bioavailability to reduce lipid absorption for hyper-lipidemics, those at risk for cardiovascular disease, or those who suffer from metabolic syndrome (McClements et al. 2008; Meynier and Genot 2017). Possible ways to modify the bioavailability of lipophilic components include: controlling the characteristics of lipid molecules, 
lipid droplet size, crystallinity of the lipid phase, interfacial properties and/or food matrix composition (McClements et al. 2008). It is also important to distinguish factors affecting lipolysis rate compared to extent of lipid digestion. The rate of digestion can be affected without changing the final amount absorbed, i.e., transit time of chyme. Whereas, modifying the extent of digestion affects the final amount absorbed, i.e., bioaccessibility (McClements et al. 2008).

Overall bioavailability of bioactive compounds encapsulated in lipid nanoparticles can be calculated according to the equation shown in Figure 2.4. $\mathrm{F}_{\mathrm{C}}$ is the fraction of the active micronutrient form when food is ingested. $F_{B}$ is the fraction released from the food matrix and solubilized within GI fluids. $\mathrm{F}_{\mathrm{A}}$ is the absorbed fraction of the bioactive that crosses the mucosal layer and epithelium cells (Singh et al. 2009). Transcellular or paracellular are the major pathways for intestinal absorption of glucose, peptides, as well as, lipophilic and hydrophilic drugs (Patel 2011; Karasov 2017). Finally, $F_{M}$ represents the unmetabolized fraction that remains bioactive after chemical transformations within the GI tract (i.e., first-pass metabolism) and enters systemic circulation (Singh et al. 2009). First-pass metabolism (or elimination) is the process in which a drug/bioactive is metabolized between the site of administration and incorporation into systemic circulation, thereby reducing its plasma concentration (Pond and Tozer 1984; Yao et al. 2015). The major site of first-pass elimination is the liver, but other sites that may affect bioavailability include the GI tract, blood, vascular endothelium, and lungs (Pond and Tozer 1984). 


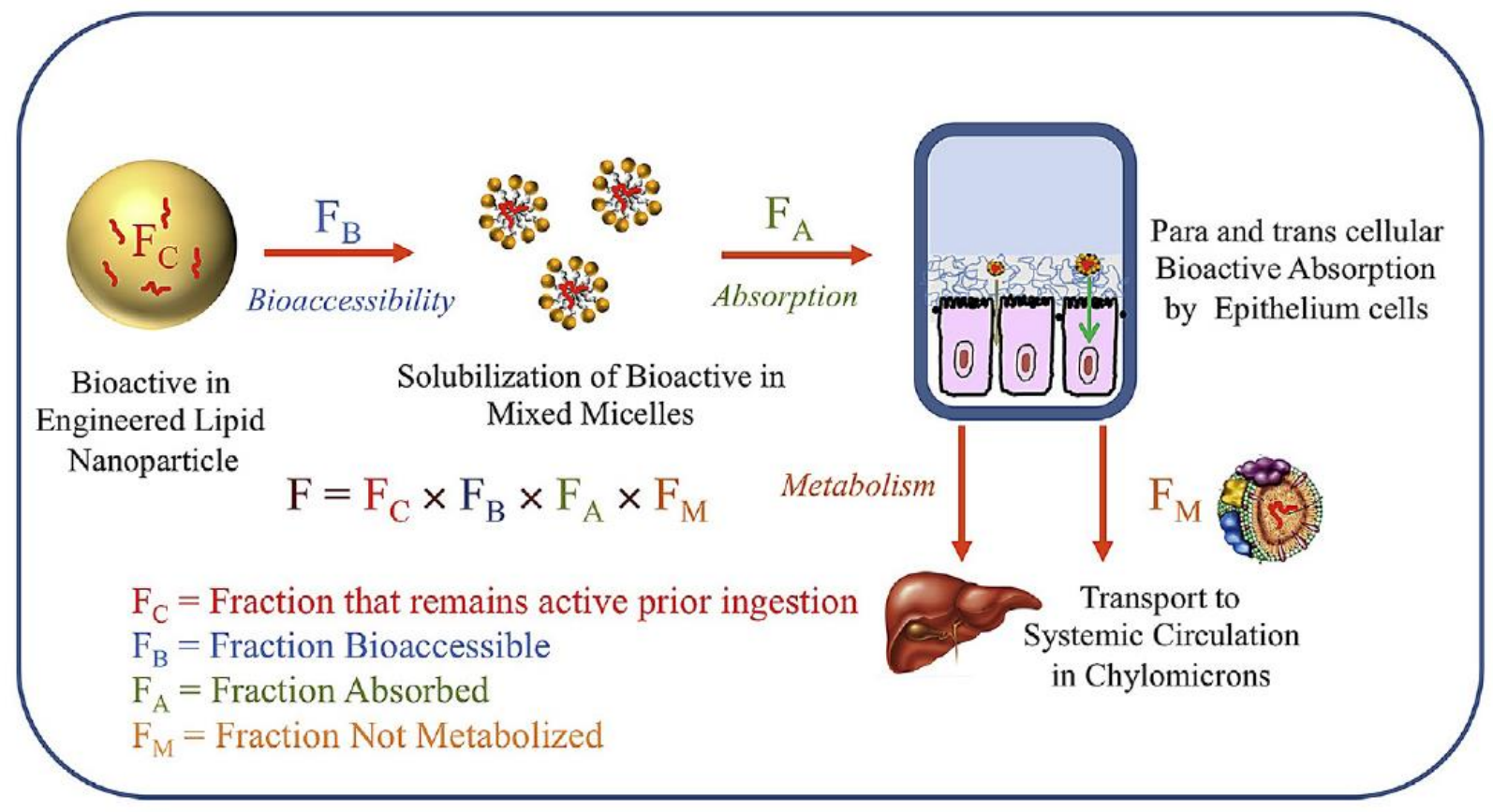

Figure 2.4 Overall bioavailability of a bioactive compound encapsulated in a lipid nanoparticle (Joye et al. 2014).

\subsection{Factors Affecting Lipid Digestion}

\subsubsection{Emulsion Droplet Size}

Oil droplet size of emulsions determines the interfacial surface area available for enzyme activity (Verkempinck et al. 2018). Smaller droplet sizes $(<1 \mu \mathrm{m})$ correspond to greater interfacial surface area available for lipase adsorption, thus faster lipolysis rates and higher lipid digestibility is typically observed. Conversely, larger droplet sizes $(>10 \mu \mathrm{m})$ result in less surface area and confer a slower lipolysis rate (Salvia-Trujillo et al. 2013a; Zhang and McClements 2016; Guo et al. 2017; Salvia-Trujillo et al. 2017). Salvia-Trujillo et al. (2013) found significant differences in the rate and extent of in vitro lipolysis between emulsions with smaller droplet diameters $(<0.4$ $\mu \mathrm{m})$ compared to larger droplet diameters $(\sim 23 \mu \mathrm{m})$. Smaller droplet size emulsions demonstrated higher rates of digestion and greater bioaccessibility of FFAs. Emulsions with larger droplets were more susceptible to coalescence; while, emulsions with smaller droplets typically flocculated. 
Overall, the rate and extent of lipolysis was inversely associated with droplet size, which was attributed to greater TAG exposure to lipase (Salvia-Trujillo et al. 2013a). Droplet size significantly influenced gastric and pancreatic lipolysis shown in healthy human adults. An increase in initial particle size (i.e., $0.7 \mu \mathrm{m}$ to $10 \mu \mathrm{m}$ ) lowered gastric lipolysis from $35 \%$ to $16 \%$ and pancreatic lipolysis decreased from $73 \%$ to $46 \%$ when initial particle size increased from 0.7 to $10 \mu \mathrm{m}$ (Borel et al. 2018).

Emulsion stability throughout digestion needs to be considered in conjunction with particle size (Verkempinck et al. 2018). A recent in vitro study by Guo et al. (2017), showed acid-stable emulsions remain homogenous; while acid-unstable emulsions, in gastric and duodenal conditions, exhibited creaming and flocculation. According to Meynier and Genot (2017), acid-unstable emulsions phase separate in gastric conditions forming an oil phase on top of the bolus causing the aqueous layer to empty the stomach first (Meynier and Genot 2017). An in vivo study by Marciani et al. (2007) found subjects fed acid-unstable meals experienced exponential gastric emptying curves, higher release of CCK, more gallbladder contractions, and decreasing post-prandial appetite. Conversely, acid-stable meals had more rapid, linear emptying curves, with higher rates of lipolysis in the duodenum (Marciani et al. 2007). A reduction in acid-stable emulsion droplet size from $52 \mu \mathrm{m}$ to $0.3 \mu \mathrm{m}$ delayed gastric emptying in human studies by 38 min (Steingoetter et al. 2015). The relationship between droplet size and digestion rate are confounded by free surfactant concentration, aqueous phase concentration, and changes to interfacial structure (SalviaTrujillo et al. 2013a).

In vitro studies using model emulsion systems, rather than real food matrices, report increasing rates of lipolysis with smaller droplet size (Armand et al. 1992; Ythier et al. 2003). While, in vivo studies found initial droplet size does not affect the extent of lipolysis because of 
the relatively long time-scale of digestion which ensures all lipids are digested, regardless of differences in initial lipolysis rates (McClements et al. 2008; Borel et al. 2018). Furthermore, phospholipid-stabilized emulsion droplets reached a final mean diameter of 10-20 $\mu \mathrm{m}$ in human subjects irrespective of initial droplet size (Bauer et al. 2005; Back 2008; Borel et al. 2018). Nanoscale emulsions digested slower compared to conventional emulsions due to much higher emulsifier-to-oil ratio required, resulting in thicker interfacial protein coating; therefore, lipolysis rate cannot be predicted using only particle size (Lee et al. 2011).

\subsubsection{Emulsifier Type}

Emulsifier structure influences the gastrointestinal fate of emulsions by modifying lipase activity (Singh and Ye 2013; McClements and Gumus 2016). Surfactants at the oil-in-water droplet interface may inhibit lipase via steric hindrance, interfacial complex formation, direct interaction with lipase, and interaction with other digestive compounds (i.e., bile) (Mun et al. 2007; Back 2008; Golding and Wooster 2010; Speranza et al. 2013). Commonly used food-grade emulsifiers include: low molecular weight (LMW) surfactants, proteins, phospholipids, and polysaccharides (Hasenhuettl 2008; Kralova and Sjöblom 2009). Different conformations adopted by surfactants at an emulsion interface affect the surface loads (Figure 2.5). For example, LMW surfactants compared to polysaccharides form smaller emulsion droplets under the same concentration and homogenization conditions (McClements and Gumus 2016). The surface load of common emulsifiers increases for: LMW surfactants < globular proteins < flexible proteins < polysaccharides (Ozturk et al. 2014, 2015). Generally, emulsion droplet size decreases with increasing emulsifier concentration (McClements and Gumus 2016). 


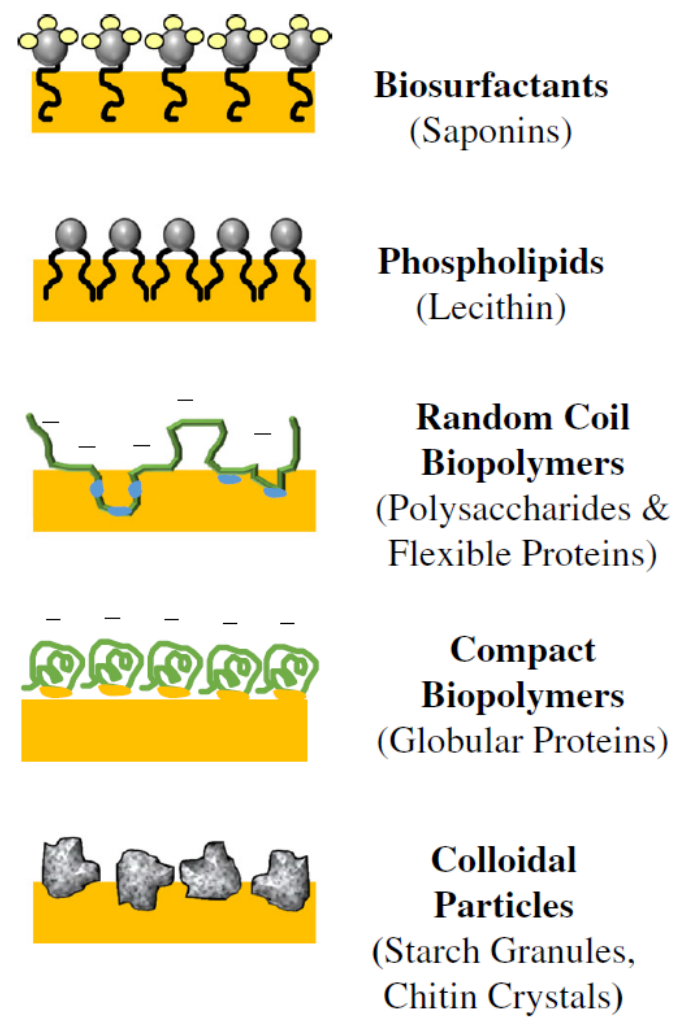

Figure 2.5 Common natural surfactants used to stabilize emulsions (McClements and Gumus 2016)

Emulsifiers, primarily used in food emulsions, aid in achieving shelf-life stability against coalescence and flocculation destabilizing reactions. Subsequently, emulsifier choice also influences emulsion stability during lipid digestion (Guo et al. 2017). As previously mentioned, emulsion instability (i.e., increase in droplet size) decreases interfacial surface area, slowing digestion rate, impacting fat absorption and modifying hormones released that play a central role in satiety. Particle size changes in emulsions are modified during digestion, which are attributed to: emulsion destabilization mechanisms (i.e., flocculation, coalescence, Ostwald ripening), enzymatic degradation, release of lipolysis products, and/or solubilization of lipid molecules into the aqueous phase (McClements 2013). Surfactant chemistry determines its interfacial properties, such as: thickness, charge, permeability, rheology, environmental-responsiveness, and digestibility (Mason et al. 2006; McClements 2013). 
Interfacial composition and properties of emulsified oil droplets are affected by changing physicochemical and biological conditions throughout the GI tract; for example, surface-active components in intestinal fluids may competitively adsorb at the interface or co-adsorb with added emulsifiers (McClements 2013). Numerous studies show emulsion droplets coated with non-ionic surfactants tend to be negatively-charged due to anionic impurities that adsorb to the interface (i.e., FFAs, phospholipids, small anions such as $\mathrm{OH}^{-}$from water) (Hsu and Nacu 2003; Beysseriat et al. 2006; Mun et al. 2006; Qian et al. 2012). Multilayer formation occurs when surface-active molecules released in the intestinal components adsorb on top of the original interfacial materials. Furthermore, emulsifiers can be digested or degraded due to the acidic environment in the gastric compartment or due to enzymatic activity (McClements 2013). In this literature review, the effect of emulsifier structure on lipid digestion will be compared for LMW surfactants (e.g., Spans, Tweens, phospholipids/lecithins), proteins, and polysaccharides.

LMW surfactants decrease interfacial tension more than proteins and polysaccharides (Wilde et al. 2004). An in vitro study by Speranza et al. (2013) demonstrated that the chain length and hydrophilic-lipophilic balance (HLB) value of emulsifiers influence rate of lipolysis and lipid bioaccessibility. Overall, decreased lipolysis rates in oil-in-water $(\mathrm{o} / \mathrm{w})$ emulsions were observed with increasing HLB values of surfactants and decreasing aliphatic chain lengths; while, lipid bioaccessibility was positively-correlated with HLB value and negatively-correlated with aliphatic chain length (Speranza et al. 2013). Lipolysis rates increased with surfactants of longer aliphatic chain lengths which was attributed to inefficient molecular packing of surfactants with longer aliphatic chains at the interface, thus allowing them to be easily displaced providing interfacial access to lipase (Israelachvili 2011; Speranza et al. 2013). The negative correlation between HLB value and lipolysis rates was attributed to the fact that hydrophilic surfactants reside primarily in 
the aqueous phase at the interface thereby limiting lipase access, as lipases are water-soluble proteins that approach the interface from the aqueous phase (Singh and Ye 2013; Speranza et al. 2013). Lipid bioaccessibility increased with increasingly hydrophilic surfactants as molecules with high HLB values may act as effective detergents which solubilize sn-2 MAGs generated in situ and promote continual lipase activity. On the other hand, increasing aliphatic chain length resulted in decreasing lipid bioaccessibility as chain length is negatively-correlated with HLB values (Reis et al. 2008; Speranza et al. 2013)

A study by Verkempinck et al. (2018) compared the physicochemical stability of emulsions stabilized with Tween 80 (HLB 15) or sucrose esters (HLB 8, 11, and 16). Tween 80-stabilized emulsions remained stable in simulated gastric conditions and maintained smaller particle sizes $(1.37-1.50 \mu \mathrm{m})$, while sucrose ester-stabilized emulsions exhibited a drastic increase in particle size (40.84-77.58 $\mu \mathrm{m})$ (Verkempinck et al. 2018). Sucrose esters interact with proteins (including digestive enzymes - i.e., pepsin) via hydrogen bonds between non-charged polar side groups of proteins and hydrophilic regions of sucrose esters. Sucrose esters with high HLB values tend to interact the most strongly with proteins (Nelen et al. 2015; Verkempinck et al. 2018). According to Marciani et al. (2006), Tween-stabilized emulsions are acid-stable; while, Span-stabilized emulsions are acid-unstable. Sucrose esters are also considered acid-unstable surfactants (Rao and McClements 2011; Verkempinck et al. 2018). Tween 80-stabilized emulsions had a significantly higher rate and extent of lipolysis compared to sucrose ester-stabilized emulsions. This was attributed to greater stability in gastric conditions allowing it to maintain smaller initial droplet size and larger surface area for lipase adsorption at the start of the simulated intestinal phase (Verkempinck et al. 2018). The rate of lipolysis for sucrose ester-stabilized emulsions increased with increasing HLB values, likely due to emulsion instability in acidic gastric conditions and 
stronger interactions with pepsin, leading to flocculation of emulsion droplets and decrease in available interfacial area for lipase (Verkempinck et al. 2018).

The amphiphilic structure of phospholipids consists of a glycerol backbone, two fatty acids that act as the non-polar hydrophobic tail, and a phosphoric acid moiety (i.e., polar hydrophilic head group) which reduces surface tension (McClements and Gumus 2016). Phospholipids form three common structures upon interfacial adsorption: (1) phosphatidylcholine (PC) forms lamellar structures with well-ordered mono- and bi-layers, allowing it to form stable nanoemulsions (100$300 \mathrm{~nm}$ ) and liposomal vesicles; (2) phosphotidylethanolamine (PE) forms reverse hexagonal structures that are more difficult to arrange at the interface; (3) lysophosphatyidylcholine (LPC) and lysophosphatidylethanolamine (LPE) exhibit enhanced hydrophilicity and form hexagonal clusters (Figure 2.6) (van Nieuwenhuyzen and Szuhaj 2002). The most commonly-used phospholipid-based commercial emulsifiers are lecithins sourced from soybean, egg, milk, rapeseed, canola, cottonseed, or sunflowers (Bueschelberger et al. 2015). Lecithins are usually a combination of phospholipids and lipophilic components such as TAGs, glycolipids, and sterols (Guiotto et al. 2013). The most common phospholipids in commercial lecithin include: phosphatidylcholine, phosphatidylethanolamine, phosphatidylinositol, and phosphatidic acid. The ratio of these phospholipids present impacts emulsion formation; for example, phospholipid ingredients composed of more PC produce smaller emulsion droplets (McClements and Gumus 2016). 


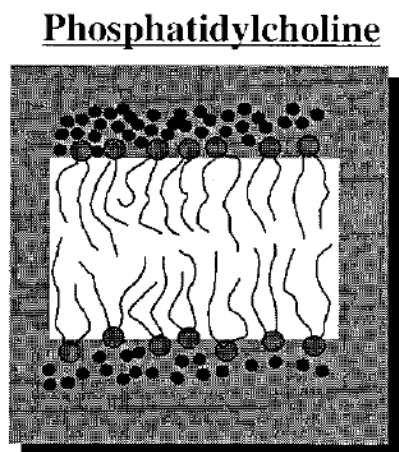

Lamellar phase
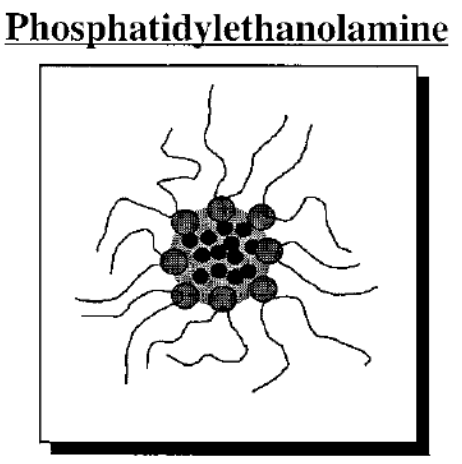

Reversed hexagonal phase

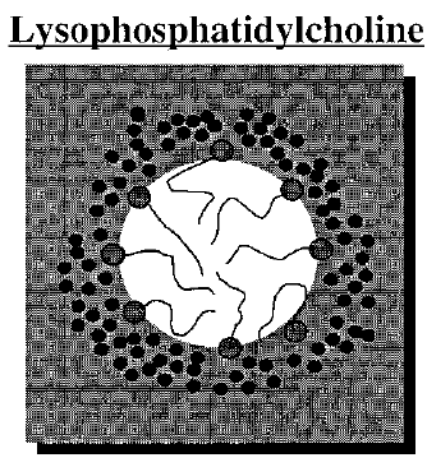

Hexagonal phase

Figure 2.6 Common phospholipid structures at an emulsion interface (van Nieuwenhuyzen and Szuhaj 2002).

Lecithin is a zwitterionic surfactant that stabilizes emulsions via electrostatic repulsive forces, while steric forces do not play a significant role (Gamon et al. 1989; Mantovani et al. 2013). A study by Torcello-Gomez et al. (2011) compared the rate and extent of in vitro lipid digestion by emulsions stabilized with lecithin (Asolectin) or a non-ionic synthetic surfactant (Pluronic F68). Pluronic F68 is a triblock co-polymer that forms bulky interfacial layers that primarily prevents coalescence via steric repulsion (Torcello-Gómez et al. 2011). Pluronic F68 was more effective at producing smaller droplets during homogenization, as well as, preventing aggregation after homogenization compared to lecithin. This was attributed to lower elasticity of the Pluronic F68 interfacial layer which creates highly-deformable droplets (Torcello-Gómez et al. 2011). The addition of lipase and bile resulted in a large increase in droplet size for the lecithin-stabilized emulsion, whereas the Pluronic-stabilized emulsion maintained a large population of small droplets. This suggests that lecithin was almost completely displaced by lipase/bile extract, promoting droplet coalescence; while, the presence of smaller droplets in the Pluronic-stabilized emulsion indicates less lipolysis had occurred (Torcello-Gómez et al. 2011). Overall, Pluronicstabilized emulsions demonstrated a lower rate and extent of lipolysis compared to lecithinstabilized emulsions. Pluronic-stabilized emulsions were more resistant to lipolysis and interfacial 
displacement from bile extract and lipase (Torcello-Gómez et al. 2011). This agrees with a previous study that reports Pluronic-stabilized emulsions are more resistant to destabilization by bile salts and better at inhibiting lipase adsorption than phospholipid-stabilized emulsions (JódarReyes et al. 2010).

Orogenic displacement is a three-stage mechanism that describes protein displacement from an interface (i.e., gas-liquid, liquid-liquid, or solid-liquid) by LMW surfactants. In the first stage, surfactants adsorb at weak points in the interfacial protein film and increase the local surface pressure without affecting the thickness of the protein film; thus, compressing the surrounding protein film. In the second stage, growth of surfactant domains decreases protein film area which causes film thickness to increase. Lastly, sufficiently high surface pressure causes breakdown of the protein network, allowing the formation of a continuous surfactant phase which completely displaces protein from the interface (Mackie et al. 2000). Based solely on this mechanism of orogenic displacement, bile is incapable of exerting enough surface pressure to displace interfacial phospholipids. However, the unique structure of bile disrupts interfaces beyond theoreticalexpectations and promotes emulsification and lipase adsorption (Wickham et al. 2002; Chu et al. 2010; Wilde and Chu 2011). Unlike classical surfactant structures that consist of a hydrophobic head group and hydrophilic tail, bile salts exhibit facial amphiphilicity that consists of a rigid steroidal backbone with a hydrophobic and hydrophilic face (Figure 2.7) (Maldonado-Valderrama et al. 2011; Sarkar et al. 2016b). Bile alters surface pressures of adsorbed lipid layers via ionic interactions with lipid head groups, changing its conformation upon adsorption, and increasing surface pressure to disrupts lipid monolayers (Dreher et al. 1967). Although LMW surfactants are widely used in industry, bile easily displaces them limiting their role in in vitro lipid digestion (Li 
and Mcclements 2010; Maldonado-Valderrama et al. 2011; Torcello-Gómez et al. 2011, 2012; Pinheiro et al. 2013; Zhang et al. 2015).
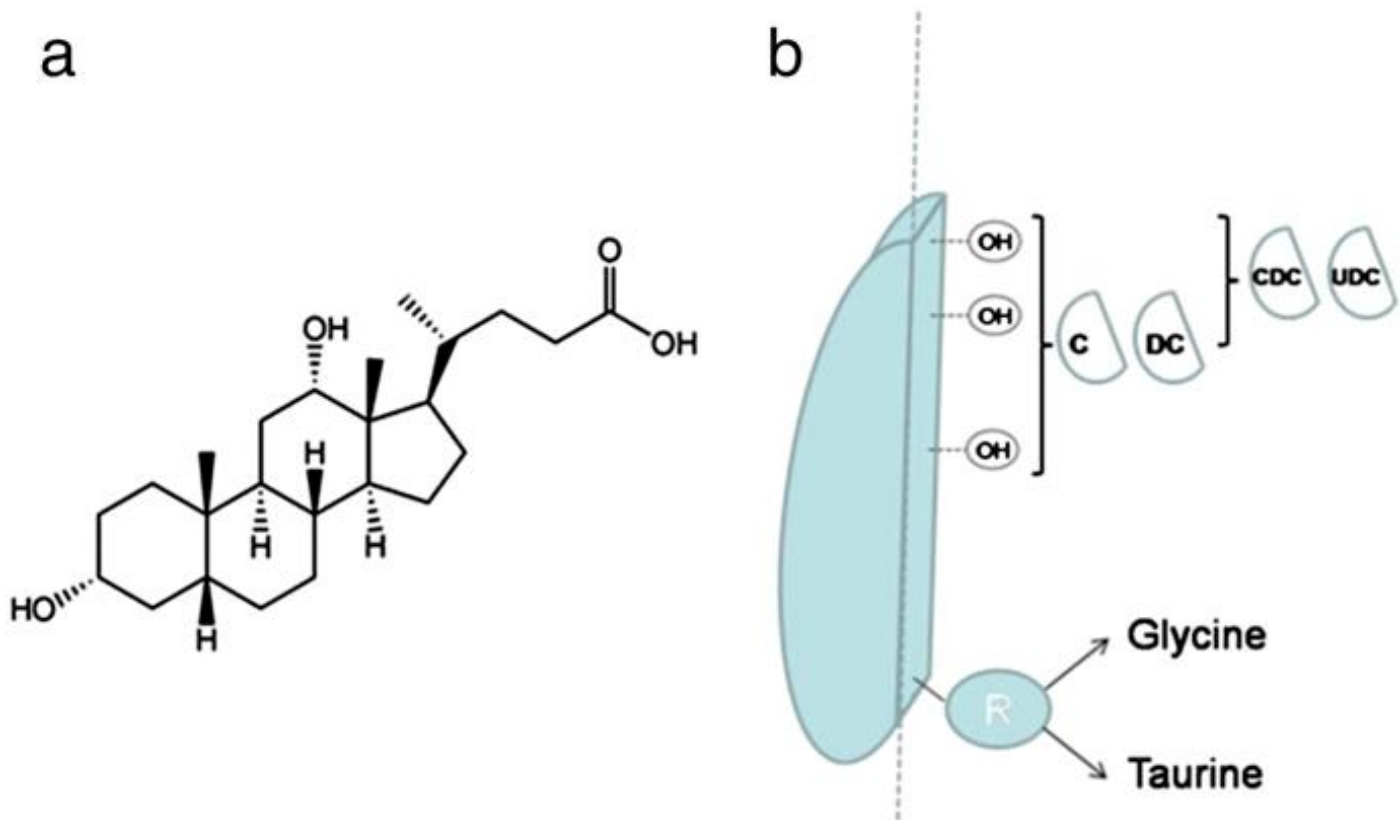

Hydrophobic Hydrophilic

Figure 2.7 (a) Deoxycholic acid and (b) schematic representation of facial amphiphilic structure of bile salts (i.e., polar hydroxyl groups on concave side, methyl groups on convex side) (Maldonado-Valderrama et al. 2011).

Proteins adsorb to emulsion interfaces reducing interfacial tension and free energy of the system by forming viscoelastic networks at the oil-water interface (Wilde and Chu 2011; Singh and Ye 2013). Globular and random coil tertiary structures of proteins are most commonly-sought for emulsifiers used in the food industry (Dickinson 2003). Globular proteins have compact spherical structures that orient non-polar groups away from the aqueous phase into their core (i.e., whey, soy, and egg proteins). While, random coil proteins have open, flexible structures with ordered regions of helical or sheets (i.e., casein and gelatin) (Tcholakova et al. 2008; McClements and Gumus 2016). Casein proteins, which have more open structures compared to rigid globular proteins, rapidly undergo conformational changes upon adsorption (Singh and Ye 2013). These 
conformational changes are controlled by the hydrophobic effect which drives hydrophilic groups to reside in the aqueous phase and hydrophobic groups to reside in the oil phase (McClements and Gumus 2016). Unfolding of globular proteins exposes free sulfhydryl groups and disulfide bonds which influences emulsion stability (McClements et al. 1993; Monahan et al. 1993; Wierenga et al. 2006; Zhai et al. 2011). For example, the native structure of $\beta$-lactoglobulin is resistant to pepsin but becomes susceptible to proteolysis when it absorbs to an interface due to conformational changes exposing peptide bonds (Singh and Sarkar 2011). Proteins are susceptible to the hydrolytic activity of proteases in the stomach and small intestine, which weakens the interfacial protein network and decreases its resistance to displacement by surfactants and bile salts (Macierzanka et al. 2009; Mackie and Macierzanka 2010; Malaki Nik et al. 2010; Maldonado-Valderrama et al. 2010). Furthermore, bile salts have lower equilibrium interfacial tension than proteins allowing them to disrupt viscoelastic protein films, likely due to interactions between ionic groups of bile salts and polar headgroups of proteins (Gunning et al. 2004; Maldonado-Valderrama et al. 2008).

The two most common milk proteins used as emulsifiers are globular whey proteins and flexible caseins (Singh and Ye 2013). Upon adsorption, whey proteins undergo partial interfacial denaturation to form compact interfacial layers $\sim 2 \mathrm{~nm}$ thick (Dalgleish 2002; Singh 2005). Caseins, which lack secondary and tertiary structures, unfold quickly and form extended interfacial layers $\sim 10 \mathrm{~nm}$ thick (Dalgleish 2004). Caseins are disordered and easily hydrolyzed by pepsin compared to whey proteins, which are more compact (Singh and Ye 2013). The influence of various emulsifiers on in vitro digestibility using pancreatic lipase demonstrated sodium caseinatestabilized emulsions were more prone to flocculation; while, whey protein-stabilized emulsions were prone to coalescence (Mun et al. 2007). Flocculation of sodium caseinate-stabilized emulsions was attributed to the formation of a thick interfacial layer, allowing sufficient caseinate 
monolayer to remain at the interface during digestion, compared to rigid globular whey proteins that form thin interfacial layers (Dickinson and McClements 1995; Mun et al. 2007). In addition, sufficient monolayer coverage for protein-stabilized emulsions is necessary to prevent bridging flocculation during homogenization, in which proteins are shared between droplets. Bridging flocculation is common with aggregated milk protein products, such as calcium caseinate or micellar casein (Singh and Sarkar 2011).

The effects of sodium caseinate (flexible protein), lactoferrin (globular protein), and Tween 80 (non-ionic surfactant) on the in vitro GI fate of o/w emulsions were compared (Zhang et al. 2015). The formation of larger droplets in the oral phase, observed in protein-stabilized emulsions, was due to bridging or depletion flocculation by mucin (Vingerhoeds et al. 2005, 2009; Sarkar et al. 2009). In the gastric stage, a large increase in average particle size was observed for proteinstabilized emulsions (Zhang et al. 2015). Tween 80-stabilized emulsions demonstrated better resistance to droplet aggregation compared to caseinate- and lactoferrin-stabilized emulsions which have proven to be highly unstable in gastric conditions (Li et al. 2012; Shimoni et al. 2013). Sodium caseinate and lactoferrin instability under gastric conditions occurs because of: (1) pepsin hydrolysis of adsorbed proteins, (2) changes in $\mathrm{pH}$ and ionic strength shielding electrostatic repulsion, and/or (3) biopolymers (i.e., mucin) which promote flocculation (Singh et al. 2009; Singh and Sarkar 2011). Caseinate-stabilized emulsions had lower lipolysis rates compared to Tween 80- and lactoferrin-stabilized emulsions due to extensive flocculation in the gastric phase, which agrees with a previous study (Li et al. 2012; Zhang et al. 2015). Other studies have confirmed emulsions that are highly aggregated upon reaching the small intestine result in slower digestion rates (Golding and Wooster 2010; Golding et al. 2011). 
Polysaccharides form thick interfacial layers that prevent droplet aggregation via steric hindrance (Dickinson 2003; McClements 2004a). Protein interfacial layers (<10 nm) are thinner than polysaccharide layers $(>10 \mathrm{~nm})$ and therefore do not rely as heavily on steric repulsion to prevent aggregation. Stronger steric repulsive forces are achieved using high MW proteins with extended structures, such as casein (Dickinson 2003, 2010; McClements 2004b; Lam and Nickerson 2013). However, protein-stabilized emulsions are susceptible to flocculation in high salt concentrations or near their isoelectric point. For example, a decrease to a $\mathrm{pH}$ lower than 2 , typically observed in gastric conditions, causes partial or full charge reversal in proteins. Furthermore, high ionic strength shields electrical charges on interfacial proteins leading to droplet aggregation (Dickinson 1994; McClements 2004b; Singh and Ye 2013). Polysaccharides require much greater surface loads compared to proteins. Typically, a 1:1 emulsifier-to-oil ratio is required for polysaccharides; while, whey proteins only require a 1:10 ratio (Xiang et al. 2015). As the primary mechanism of stabilization for polysaccharides is steric repulsion, this promotes stability over a wide range of environmental conditions compared to protein-stabilized emulsions (Dickinson 2003; McClements 2004a). For example, gum arabic-stabilized emulsion droplets are stable to flocculation between $\mathrm{pH} 3-9$, salt conditions between 0-500 $\mathrm{mM} \mathrm{NaCl}$ and $0-25 \mathrm{mM}$ $\mathrm{CaCl}_{2}$, and temperatures between $30-90^{\circ} \mathrm{C}$ (McClements and Gumus 2016).

Lundin and Golding (2009) compared the ability of three different emulsifiers (i.e., phospholipids, whey protein isolate (WPI), and a mixed interface ( $1 \% \mathrm{wt}$. sodium caseinate, $0.25 \%$ wt. monoglyceride)) to modulate in vitro lipid digestion. Phospholipid- and WPI-stabilized emulsions had smaller droplets in the simulated intestinal conditions; while, the mixed interface emulsion exhibited a drastic increase in droplet size (Lundin and Golding 2009). The pH-stat method measured lipolysis kinetics and observed the rates in this order: phospholipid > WPI > 
mixed interface (Lundin and Golding 2009). The pH-stat method measures the amount of alkali titrated into simulated chyme to maintain neutral $\mathrm{pH}$ after addition of intestinal fluids containing lipase, bile salts and $\mathrm{CaCl}_{2}$ (Yang and $\mathrm{McClements} \mathrm{2013).} \mathrm{The} \mathrm{lowest} \mathrm{rate} \mathrm{of} \mathrm{lipolysis} \mathrm{for} \mathrm{mixed}$ interface was attributed to an increase in droplet size caused by emulsion instability in gastric conditions (Lundin and Golding 2009). However, it is important to note that the pH-stat method does not account for peristalsis. The presence of shear would re-emulsify and break-up flocculating oil droplets and facilitate further lipolysis; thus, the static in vitro digestion kinetics were likely underestimated (Carlson et al. 1966; Carey et al. 1983; Lundin and Golding 2009).

\subsubsection{Oil Type}

\subsubsection{Naturally-occurring vs. processed}

Food matrices are complex, heterogeneous combinations of solids, liquids and/or gases that may either occur naturally or form as a result of processing and formulation (Guo et al. 2017). Common examples of naturally-occurring lipid structures are illustrated in Figure 2.8. Muscle food TAGs are stored in adipocytes in adipose tissue (Figure 2.8A) (Meynier and Genot 2017). Fat globules in raw milk have a TAG core stabilized by a tri-layer membrane of phospholipids, sphingolipids, cholesterol and proteins (Figure 2.8B) (Lopez 2011; Raynal-Ljutovac et al. 2011). TAGs in egg yolk consists of a TAG core surrounded by a monolayer of phospholipids, proteins and cholesterol (Figure 2.8C) (Meynier and Genot 2017). Lipids in plants, nuts, and seeds are stored in oil bodies comprised of oil droplets surrounded by a phospholipid monolayer with embedded oleosin proteins (Figure 2.8D) (Singh and Ye 2013). TAGs found in animal-derived fats predominantly contain a saturated FA at the $s n-1$ position and an unsaturated FA at the $s n-2$ position. TAGs from seed oils have primarily polyunsaturated FA at the $s n-1$ position; while, 
vegetable oil TAGs primarily contain a saturated FA at the $s n-1$ position (Karupaiah and Sundram 2007).

A- Adipocytes and cell membranes

in meat, fish and muscle foods

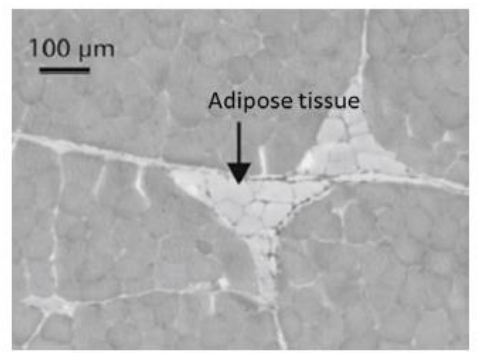

B- Milk fat globules

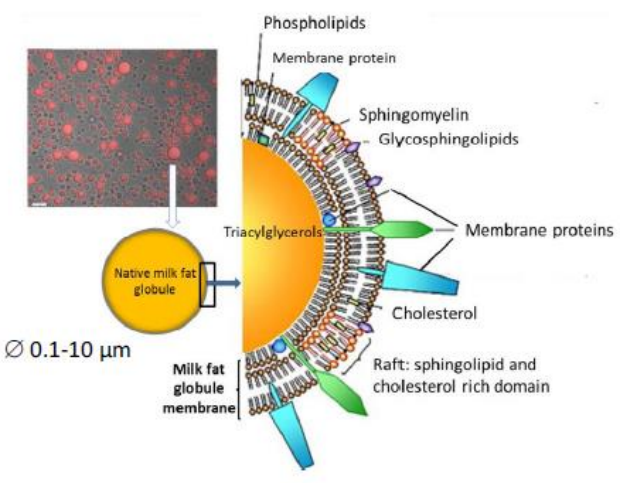

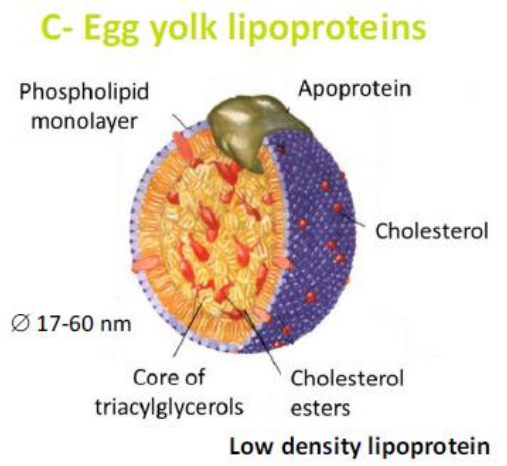

D- Oleosomes of oilseeds

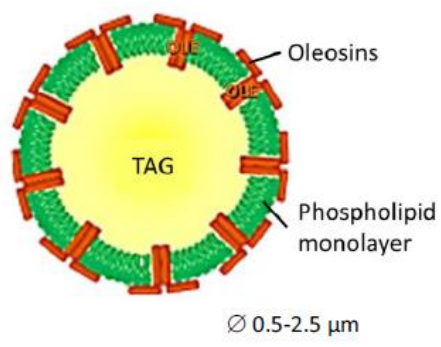

E- Vegetable cells

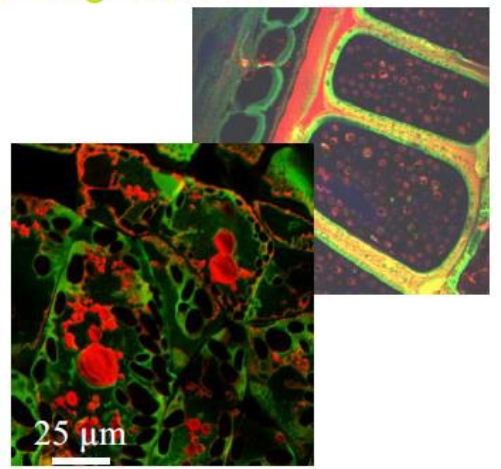

Figure 2.8 Structures of naturally-occurring lipids in food (Meynier and Genot 2017)

In foods, lipids are used as either bulk ingredients during food preparation (i.e., frying oil) or ingredients in processed foods (i.e., yogurt, cheese) (Guo et al. 2017). Lipids in processed foods exist within water-in-oil emulsions (i.e., margarine, butter) or oil-in-water emulsions (i.e., milk, salad dressing) (Singh and Ye 2013; McClements 2018). Solid dispersions of lipids are present in baked goods and comminuted meat products (Meynier and Genot 2017). Food structure not only contributes to texture, sensory properties, shelf-life and stability; but it also modifies lipid digestion. For example, fat bioaccessibility is lower in whole peanuts compared to peanut butter and peanut oil (Levine and Silvis 2010; Nyemb et al. 2014, 2016). Lipids in native oil bodies are 
partially protected from lipolysis by surrounding cellular structures that resist GI conditions (McClements 2018). Almonds contain 50\% oil bodies by weight; however, mastication is only able to release $\sim 10 \%$ of the oils stored in raw and roasted almonds, while the cells remain mostly intact (Grundy et al. 2015, 2016b).

In a study by Grundy et al. (2016), the rate and total lipid bioaccessibility of in vitro lipid digestion of isolated almond cells was significantly lower than isolated oil bodies at 22\% and 69\% hydrolysis, respectively (Grundy et al. 2016a). The decreased bioaccessibility of almond cells was attributed to the cell walls which hindered diffusion of lipase into the intracellular environment to access TAG molecules (Grundy et al. 2016a). Almond cells were observed to remain intact throughout in vitro digestion for up to $20 \mathrm{~h}$ with minimal lipid digestion and release. As lipase must diffuse through the cell wall matrix in order to access lipids contained within the inner regions of almond cells, almond cells resulted in a slower rate of digestion and release of lipolytic products compared to isolated oil bodies (Grundy et al. 2016a). Thus, digestion and bioaccessibility of oilseed lipids is influenced by cell wall integrity and interfacial composition (Guo et al. 2017).

A study by De Oliveira et al. (2016) compared in vitro lipolysis of raw and pasteurized human milk. Raw milk flocculated and aggregated after $60 \mathrm{~min}$ in gastric conditions resulting in a bimodal particle size distribution (average mode diameters $=76.5 \mu \mathrm{m}, 6.5 \mu \mathrm{m}$ ); while, pasteurized milk maintained its monomodal particle size distribution for $120 \mathrm{~min}$ (average diameter $6.5 \mu \mathrm{m}$ ) (de Oliveira et al. 2016). The lower rate of gastric lipolysis for pasteurized human milk was mainly attributed to inactivation of bile salt-stimulated lipase, an endogenous maternal lipase found in breast milk (de Oliveira et al. 2016). However, the same rate of gastric emptying was used for raw and pasteurized milk; thus, the in vitro model could not account for non-homogenous gastric emptying due to emulsion destabilization, which may modulate hormonal feedback mechanisms 
and nutrient absorption (Marciani et al. 2007; Golding et al. 2011; de Oliveira et al. 2016). However, in a randomized controlled trial using pre-term infants, similar rates of lipolysis for both raw and pasteurized breast milk were observed (De Oliveira et al. 2017). Although pasteurized milk had higher milkfat globule surface area than raw milk, interfacial protection from heatinduced protein aggregates in the pasteurized milk may have offset differences in lipolytic activity (De Oliveira et al. 2017).

Milk and yogurt (i.e., liquid and semi-solid food matrices) are more susceptible to lipolysis compared to cheese (i.e., solid matrices), as their structures breakdown more readily (Lamothe et al. 2017; Mulet-Cabero et al. 2017). Solid food matrices are constructed of 3-D networks of macromolecules (i.e., proteins, polysaccharides and lipids) that obstruct diffusion of lipase limiting lipase access to oil droplet interfaces (Guo et al. 2017). For cheeses, digestibility is influenced by structural characteristics and hardness (Fang et al. 2016). For example, an in vitro study by Fang et al. (2016) found that cheddar cheese had a higher lipid bioaccessibility compared to mozzarella because of the less compact protein network (due to larger fat globules) and higher initial fat content in cheddar. Compared to cheddar, mozzarella consists of a dense and fibrous protein matrix as a result of the stretching step during processing (Fang et al. 2016).

\subsubsection{Chain Length}

Fatty acid chain length and TAG composition (i.e., fatty acid position) affect the physical properties of lipid blends and impact lipid digestion (Thilakarathna et al. 2016). For example, the lipolytic activity of both human and porcine lipase are more active on TAGs with medium chain lengths $(<\mathrm{C} 10)$ compared to TAGs containing long chain fatty acids (>C12) (Giang et al. 2016). Lipids comprised of long chain fatty acids slow down gastric emptying and the rate of lipid digestion compared to short or medium chain fatty acids (Thilakarathna et al. 2016). Shorter chain 
fatty acids are more readily absorbed compared to long chain FAs; while, medium chain fatty acids are more rapidly hydrolyzed by pancreatic lipase than long chain fatty acids (Mu and Porsgaard 2005; Wilde and Chu 2011). The rate of in vitro TAG digestion is observed to decrease with increasing fatty acid chain length: short chain TAGs $(\mathrm{SCT})>$ medium chain TAGs $(\mathrm{MCT})>$ long chain TAGs (LCT) (Saito et al. 2003; Ozturk et al. 2015; Liang et al. 2016; Zou et al. 2016). FFAs released from LCT tend to remain at the oil-water interface and restrict lipase access, while FFAs from SCT and MCT have a higher affinity for water and migrate into the aqueous phase (Sek et al. 2002; Day et al. 2010; Devraj et al. 2012; Guo et al. 2017). The rate and extent of LCT digestion depends on higher bile salt concentrations for greater solubilization; unlike digestion of SCT and MCT which are not dependent on bile concentrations (Sek et al. 2002; Guillot et al. 2006; Salentinig et al. 2011; Devraj et al. 2012; Salvia-Trujillo et al. 2013b).

According to Devraj et al. (2012), increasing calcium ion concentration more effectively promotes LCT digestion compared to increasing bile salt concentration (Devraj et al. 2012). Calcium complexes with long chain fatty acids to form insoluble calcium soaps, removing them from the droplet interface and preventing accumulation of long chain fatty acids at the droplet interface which may inhibit lipase (Scow 1988; Zangenberg et al. 2001). An increase in $\mathrm{CaCl}_{2}$ concentration in an in vitro digestion model increased the rate of FFA release of an emulsion system; however, this effect was dependent on the emulsifier type and other food components present (i.e., calcium-chelating agents and polysaccharides) (Hu et al. 2010). These findings were consistent with a study by Ye et al. (2013) that reported the addition of calcium increased the rate and extent of FFA release throughout in vitro digestion, but to varying degrees depending on the emulsifier used (i.e., whey, casein, lecithin, or Tween 20). The profiles of individual FFAs released were not affected by calcium addition (Ye et al. 2013). 
The bioaccessibility of vitamin $\mathrm{E}$ is highly dependent on its chemical form and on the surrounding food matrix, with reported values of $\alpha$-tocopherol bioaccessibility ranging from $<1 \%$ to $100 \%$ (Reboul et al. 2006; O'Callaghan and O'Brien 2010). Yang and McClements (2013) used MCT as a carrier oil for $\alpha$-tocopherol acetate, resulting in a greater rate and extent of lipid digestion compared to LCT, which was attributed to differences in water dispersibility of the FFAs generated from lipolysis. Efficient digestion of LCT requires enough bile salts and free calcium ions to remove long chain FFAs from the interface (Li et al. 2011). However, vitamin E bioaccessibility was 17\% from the MCT emulsion and 39\% from the LCT emulsion (Yang and McClements 2013). Although the rate and extent of digestion was greater for MCT, long chain FFAs are capable of forming mixed micelles more suitable for incorporating large non-polar molecules (i.e., vitamin E) (Yang and McClements 2013). In a confirmatory study by Yao et al. (2015) it was determined LCT nanoemulsions have higher bioaccessibility of vitamin E, $\beta$-carotene, and co-enzyme Q10 compared to MCT nanoemulsions; while MCT nanoemulsions had higher bioaccessibility for curcumin compared to LCT nanoemulsions (Yao et al. 2015).

\subsection{Looking Ahead: Food Nanotechnology}

Nanotechnology is defined as "the understanding and control of matter at dimensions of roughly 1-100 nanometers, where unique phenomena enable novel applications" according to the National Nanotechnology Initiative (2019). Nanoscale structures have physical, chemical, and biological properties unique compared to their macroscopic counterparts (Weiss et al. 2006). In nature, self-assembly of nanoscale structures is thermodynamically-driven to minimize overall free energy (Weiss et al. 2006). Nanotechnology is used for pathogen control, sensors, and delivery systems in foods (Weiss et al. 2006). Delivery systems transport functional ingredients to desired site of action in the human body. The functional carrier must protect the ingredients from chemical 
or biological degradation, provide controlled release for targeted delivery, and be compatible with other system components (Weiss et al. 2006).

Micronutrients often cannot be incorporated into food products due to limited solubility, stability, and sensory acceptability. Therefore, food-grade nanoparticle delivery systems are designed to encapsulate, protect, and release micronutrients (Joye et al. 2014; McClements 2014). Nanoparticles are fabricated from LMW surfactants, lipids, proteins, polysaccharides, or minerals (McClements 2014). Radius of nanoparticles $<100 \mathrm{~nm}$ has the potential to provide optical clarity, physicochemical stability, good encapsulation and release characteristics, and higher bioactivity (Sekhon 2010; Ezhilarasi et al. 2013). Particles >300 nm cannot directly transport into systemic circulation; thus generally, smaller particles undergo greater absorption (Jani et al. 1990). Nanoparticle charge may be positive, negative or neutral, depending on its composition and environment (Joye et al. 2014). Nanoparticle delivery systems can have multiple release mechanisms, such as: diffusion through intact particle, particle swelling, particle disassembly, or particle erosion (Figure 2.9) (McClements 2014). For example, digestible protein nanoparticles used for targeted delivery of micronutrients in the stomach rely on particle breakdown by protease activity. Similarly, lipid nanoparticles mainly target delivery to the intestine by lipase activity (Joye et al. 2014). Examples of lipid-based nanoparticle systems include: nano-liposomes, multiple emulsions, solid lipid nanoparticles, and nanoemulsions (Joye et al. 2014). 


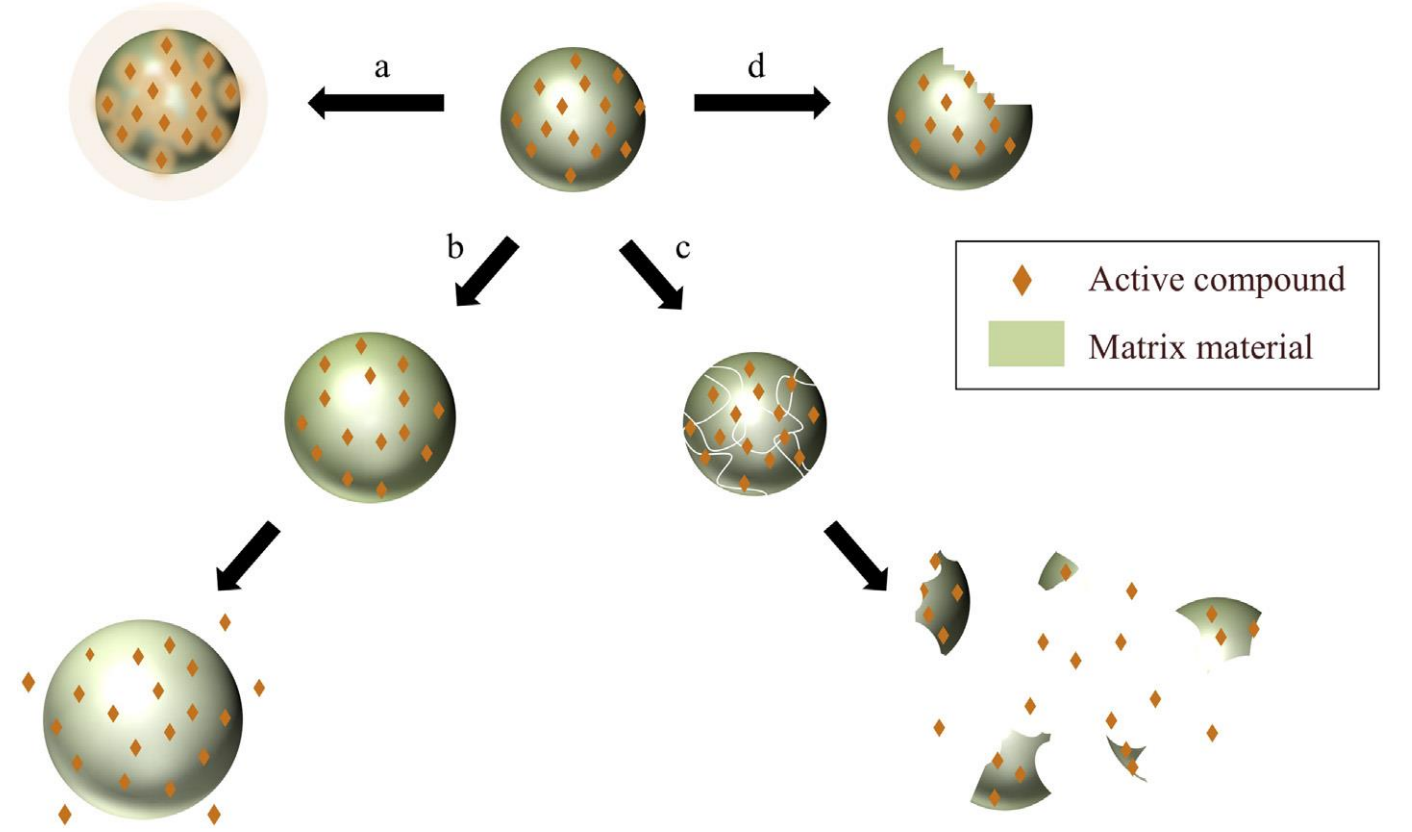

Figure 2.9 Common release mechanisms of micronutrients encapsulated in nanoparticles: (a) diffusion through intact particle matrix, (b) release through particle swelling, (c) particle disintegration, and (d) particle erosion (Joye et al. 2014).

Nano-liposomes form via the self-assembly of phospholipid in aqueous environments into spherical, bilayer vesicle structures (Taylor et al. 2005; Reza Mozafari et al. 2008; Singh et al. 2012). Phospholipids dispersed in water tend to form lamellar aggregates; however, sufficient energy will cause assembly into spherical-shaped liposomes consisting of single or multiple bilayers of phospholipids (Taylor et al. 2005; Reza Mozafari et al. 2008; Isailović et al. 2013). Both lipophilic and amphiphilic compounds may be encapsulated within the hydrophobic bilayer membranes; while, the aqueous inner core of liposomes allow encapsulation of hydrophilic compounds (Dos Santos et al. 2004; Taylor et al. 2005; Fritze et al. 2006; Zucker et al. 2009). Nano-liposomes can be formulated from natural ingredients such as soy, egg, lecithin or dairy (Joye et al. 2014).

Nano-structured multiple emulsions are emulsions within emulsions, most commonly either: oil-in-water-in-oil emulsions $\left(\mathrm{O}_{1} / \mathrm{W} / \mathrm{O}_{2}\right)$ or water-in-oil-in-water emulsions $\left(\mathrm{W}_{1} / \mathrm{O} / \mathrm{W}_{2}\right)$ (Benichou and Garti 2004, 2010). $\mathrm{W}_{1} / \mathrm{O} / \mathrm{W}_{2}$ emulsions consist of nano-sized water droplets, 
contained within larger oil droplets, dispersed in an aqueous continuous phase (Joye et al. 2014). Multiple emulsions require both oil-soluble and water-soluble emulsifiers. The first preparation step is to homogenize the water, oil, and oil-soluble emulsifier to create a $\mathrm{W}_{1} / \mathrm{O}$ emulsion. This is followed by homogenization of the $\mathrm{W}_{1} / \mathrm{O}$ emulsion with an aqueous phase and water-soluble emulsifier, but with less force than the first step so the $\mathrm{W}_{1} / \mathrm{O}$ emulsion is not disrupted. $\mathrm{W}_{1} / \mathrm{O} / \mathrm{W}_{2}$ emulsions can be used to create reduced-fat products of typical o/w emulsions (as the oil droplets are partly filled with water, lowering fat content) or for density control by incorporating denser polymers in the $\mathrm{W}_{1}$ phase to prevent gravitational separation (McClements 2012). Anthocyanins have been incorporated into $\mathrm{W}_{1} / \mathrm{O} / \mathrm{W}_{2}$ emulsions with $>80 \%$ encapsulation efficiency (Akhtar et al. 2014). But compared to conventional emulsions, multiple emulsions are difficult to prepare, more expensive, and highly susceptible to breakdown during storage or due to environmental stresses (McClements 2012).

Solid lipid nanoparticles (SLNs) are o/w emulsions where the lipid phase is partially or fully crystalline (McClements et al. 2007; Weiss et al. 2008; McClements and Li 2010). Preparation of SLNs involve creating a nanoemulsion above the melting temperature of the lipid phase, then temperature is lowered to induce nucleation and subsequent crystal growth. The solidified lipid phase delays molecular diffusion of entrapped lipophilic compounds, slowing down chemical degradation, and improving stability of entrapped compounds (McClements 2013; Joye et al. 2014). Changing the lipid physical state alters lipase accessibility to ester bonds of TAGs and its solubilization into mixed micelles (McClements et al. 2008; McClements and Li 2010). Generally, less ordered lipid crystalline phases are better for encapsulation and bioactive retention (Guri et al. 2013). A study comparing the physical state of the lipid phase (i.e., either liquid or solid) in o/w emulsions reported a higher rate and extent of lipolysis in the liquid emulsion 
compared using the pH-stat model (Bonnaire et al. 2008). Another study by Guri et al. (2013) reported increased retention time and stability of curcumin encapsulated in SLNs compared to conventional emulsions (Guri et al. 2013). SLNs may also be used as delivery systems for omega3 fatty acids, conjugated linoleic acid, phytosterols or carotenoids (McClements and Li 2010).

Nanostructured multi-layer emulsions consist of oil droplets surrounded by layers of polyelectrolytes. These layers form using a layer-by-layer (LbL) electrostatic deposition method, which is the sequential adsorption of polyelectrolytes onto surfaces of oppositely-charged colloidal particles (Weiss et al. 2006). First, a primary emulsion is formed via homogenization of oil, water, and an ionic emulsifier; then an oppositely-charged polyelectrolyte is added to produce a secondary emulsion with a two-layer interface. These sequential steps can be repeated creating multiple layers (Gu et al. 2005; Mun et al. 2005; Guzey and McClements 2006). The LbL method controls charge, thickness, permeability, and environmental responsiveness of interfacial layers. Multi-layer emulsions are typically engineered to resist environmental stresses (i.e., $\mathrm{pH}$ and ionic strength) compared to conventional emulsions with monolayer interfaces (Decher 1997; Mun et al. 2005; Guzey and McClements 2006, 2007). Bioactives can be protected by residing in the core; while, the interfacial shell can be designed for targeted release in response to environmental stimuli (Weiss et al. 2006). However, being susceptible to changes in $\mathrm{pH}$ or ionic strength may also disrupt the electrostatic interactions holding together the interfacial layers (McClements and Li 2010).

Nanoemulsions are formed via low energy (i.e., spontaneous formation due to a change in the composition or temperature of a surfactant, oil, and water mixture) or high energy methods (i.e., homogenization, ultrasonication, etc.) (Rao and McClements 2011). Low energy methods require higher surfactant concentrations. Energy intensive methods require high shear forces to disrupt the oil phase into nano-sized droplets, but less surfactant and allows for wider ingredient 
options (Joye et al. 2014). Irrespective of the method, surfactants are crucial in nanoemulsions due to the large surface area that needs stabilized compared to conventional emulsions (Ubbink et al. 2008). LMW surfactants are better suited for nanoemulsions, compared to proteins and polysaccharides, as they rapidly absorb at the interface reducing interfacial tension (McClements 2013). Bioactives contained within nanoemulsion droplets use the interface to protect them from degradation and reduce their permeation rate (Weiss et al. 2006). For example, resveratrol encapsulated within lipid nanoemulsions resulted in slower and more prolonged bioactive release (Sessa et al. 2014). Similarly, bioaccessibility of quercetin increased two-fold when incorporated into nanoemulsions compared to bulk oil (Pool et al. 2013). However, altering particle size at the nano-scale results in different physiological impacts (i.e., absorption, distribution, metabolism, excretion) which raises concerns over potential toxic effects (Hagens et al. 2007; Chaudhry et al. 2008; Bouwmeester et al. 2009; Souto et al. 2009).

Mixed nanoparticle delivery systems contain two or more types of nanoparticles each with their own unique functionality (McClements 2017). For example, a hydrophobic bioactive may be encapsulated within protein nanoparticles, which are then mixed with lipid nanoparticles (Figure 2.10). The protein nanoparticles protect the bioactive; while, the lipid nanoparticles act as a digestible TAG source to increase bioactive solubility in GI fluids (McClements 2017). This method was demonstrated using mixed curcumin- and tangeretin-loaded zein nanoparticles in two in vitro studies. Both studies demonstrated a higher bioaccessibility with increasing concentration of lipid nanoparticles due to greater bioactive solubilization capacity of the mixed micelle phase (Chen et al. 2015; Zou et al. 2016). 


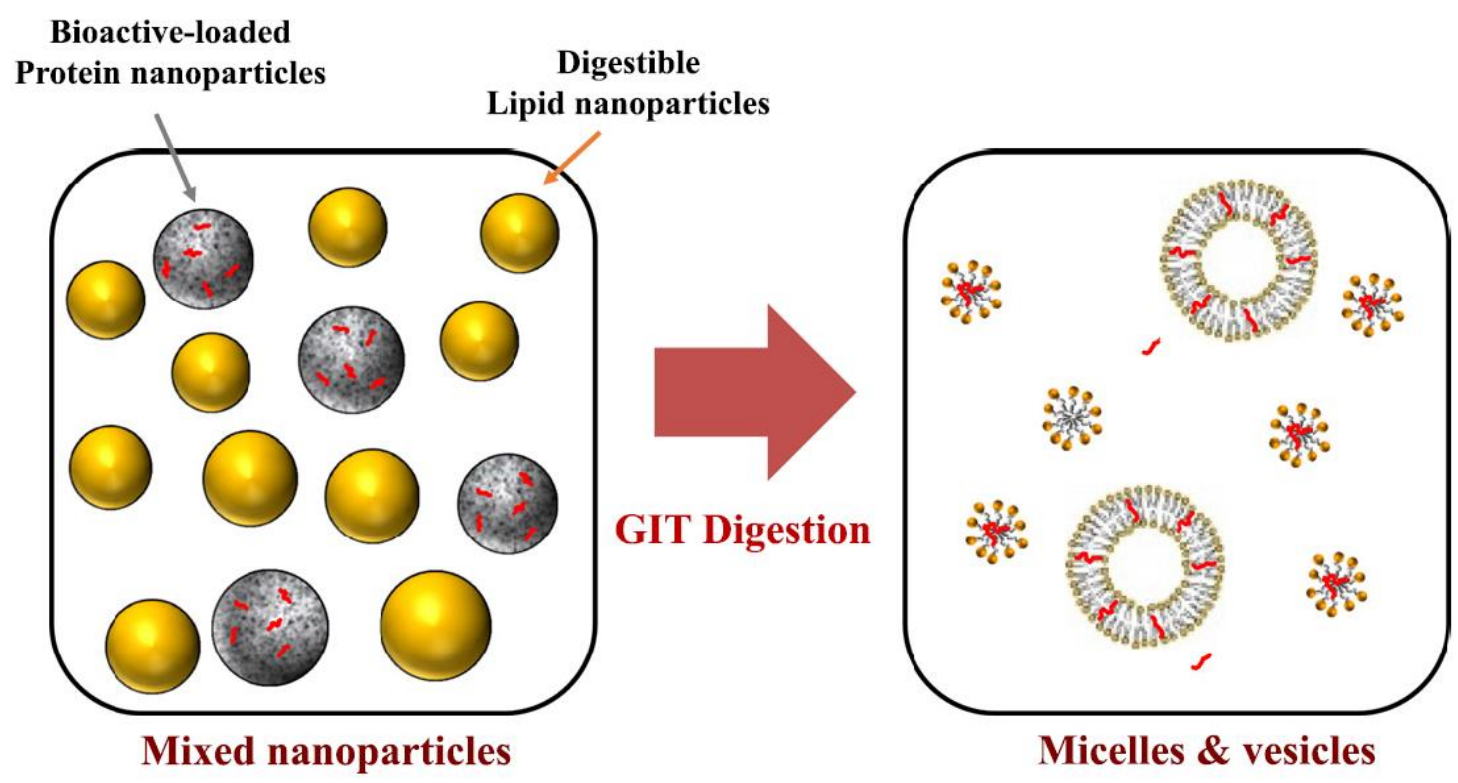

Figure 2.10 Mixed nanoparticle delivery systems with the potential to increase the bioavailability of hydrophobic bioactives in the gastrointestinal tract (GIT) (McClements 2017).

Nanolaminates consist of two or more nanometre-thick layers, physically- or chemicallybonded using the LbL deposition method (Weiss et al. 2006; Decher and Schlenoff 2012). This process is used in edible coating/film applications to act as moisture, lipid, and gas barriers (Weiss et al. 2006). Nanolaminates are also carriers for functional ingredients (i.e., anti-microbials, antibrowning agents, anti-oxidants) to increase shelf-life and quality of coated foods. Common coating materials for edible films include: polysaccharides, proteins, and lipids (Park 1999; Weiss et al. 2006).

\subsubsection{Other Novel Delivery Systems}

Colloidosomes differ from other delivery systems because its core is coated with a shell of smaller colloidal particles that may be comprised of liquid droplets, air bubbles, hydrogel microspheres, solid particles or starch granules (McClements 2012). For o/w colloidosomes, the shell colloids adsorb to the interface but protrude into the aqueous phase; thus, they have a higher affinity towards the aqueous phase compared to the oil phase (Patra et al. 2010). Colloidosomes 
are used to increase emulsion stability, density, encapsulation and protection efficiency (i.e., chemically-inert shells), and controlled release applications (McClements 2012).

Micro-clusters are flocculated oil droplets prepared using controlled aggregation of oil droplets, which may be comprised of one or more lipid types, dispersed in a continuous aqueous phase (Dickinson 2011; Mao and McClements 2011). Droplet flocculation is induced via changes in $\mathrm{pH}$, addition of electrolytes which screen electrostatic interactions, or mixing positively-charged droplets with negatively-charged droplets (McClements 2012). Micro-clustering is primarily used to control the rheological properties of emulsions because flocculated oil droplets trap the continuous aqueous phase increasing the viscosity to achieve gel or paste-like properties (Dickinson 2009; McClements 2012). Micro-clustered emulsions have demonstrated slower digestion rates compared to non-flocculated emulsions (Golding et al. 2011). However, these systems are highly susceptible to gravitational separation in dilute systems due to their larger particle sizes (Moschakis et al. 2010; McClements 2012).

Micelles and microemulsions spontaneously form when surfactants and water are mixed under specific environmental conditions (Flanagan and Singh 2006; Spernath and Aserin 2006). Micelles consist of surfactants and an aqueous phase $(\mathrm{d}<20 \mathrm{~nm})$; while, microemulsions contain an additional oil phase $(\mathrm{d}<100 \mathrm{~nm})$. These systems rely on surfactant head groups forming a hydrophilic shell and non-polar surfactant tails orienting to form a hydrophobic core. Their small particle size makes them suitable for clear product applications, such as beverages (Sagalowicz and Leser 2010; Joye et al. 2014). Microemulsions have been reported to increase the bioavailability of curcumin 20-fold due to increased solubilization in GI fluids. However, high concentrations of surfactant required for these systems may cause economic, regulatory, and product acceptability issues (Hu et al. 2012; Joye et al. 2014). 
Filled hydrogel particles are prepared by mixing an o/w emulsion with a biopolymer solution and then manipulating environmental conditions (i.e., $\mathrm{pH}$, gelling agents, molding) to induce particle formation (Pich and Adler 2007; Burey et al. 2008; Matalanis et al. 2011). These systems have higher viscosities compared to conventional o/w emulsions (McClements 2012). McClements and $\mathrm{Li}$ (2010) found calcium alginate filled hydrogel beads very effective at delaying lipid digestion, likely because lipase had to first diffuse through the gel network to access the oil droplets (McClements and Li 2010). Filled hydrogel particles are potential fat replacers; however, these particles are more expensive and difficult to prepare than conventional emulsions, and still prone to destabilization mechanisms observed for conventional emulsions (McClements and $\mathrm{Li}$ 2010; McClements 2012).

Biopolymer particles may be used as surface-active functional ingredients in emulsions and foams (Dickinson 2017). Solid particles form networks in the continuous phase acting as a structural barrier around the dispersed phase at high concentrations, or particles can adsorb to the oil-water interface forming a protective monolayer. Both occur simultaneously within multi-phase systems, where particles are attached to the interface and also dispersed in the continuous phase (Dickinson 2015). Solid particles stabilize emulsion droplets via a process termed Pickering stabilization. In Pickering emulsions, particle location at the interface depends on interactions with neighboring particles and the interfacial contact angle (Dickinson 2017). Biopolymer-based particles tend to be hydrophilic and therefore provide better stabilization to o/w emulsions. Furthermore, a monolayer of closely-packed solid particles can be fused together into a cohesive gel-like layer (Rayner et al. 2014; Sarkar et al. 2016a). Protein stabilizing particles (i.e., whey, soy, pea proteins) tend to form gel- or paste-like networks (Hoffmann and Reger 2014). 
Cellulose and chitin-based particles are resistant to digestive enzymes and gastric acids (Madadlou et al. 2016). The rate of in vitro lipid digestion for chitin-stabilized Pickering emulsions was reported to be slower than whey protein or caseinate-stabilized Pickering emulsions (Tzoumaki et al., 2013). Although emulsions stabilized by colloidal particles are highly-stable to coalescence, this Pickering stabilization mechanism is typically only applicable to emulsions with larger oil droplets $(\mathrm{d}>2 \mu \mathrm{m})$. Therefore, the large droplet size of Pickering emulsions makes them more prone to gravitational separation (Kargar et al. 2012; McClements and Gumus 2016). Despite the multitude of novel delivery systems that are emerging with unique functionalities (Figure 2.11), the most widely-used delivery system at present are still conventional o/w emulsions due to ease of preparation (McClements and Li 2010).

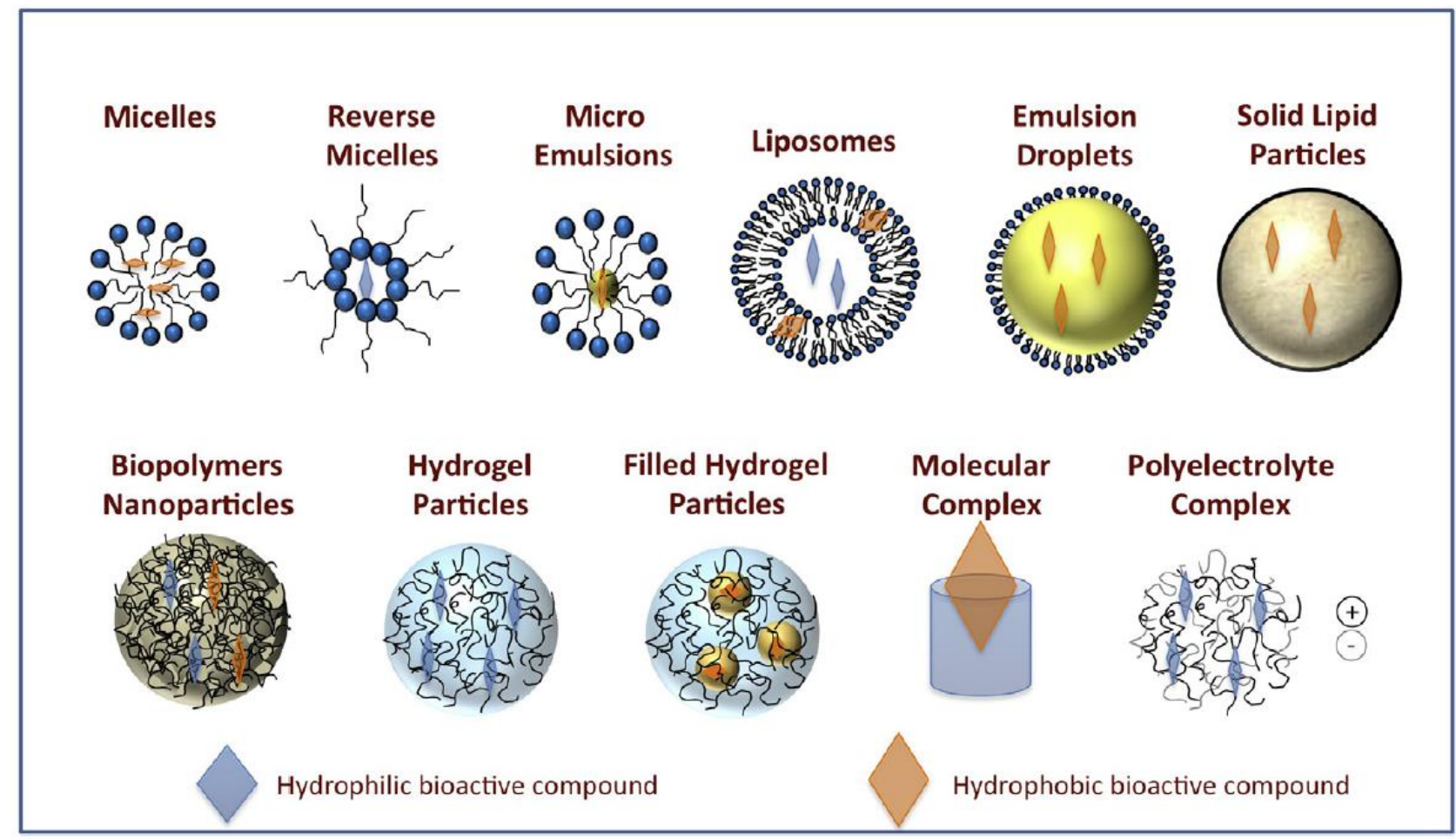

Figure 2.11 Various novel delivery systems that have potential to modify lipid digestion (Joye et al. 2014). 


\subsection{Conclusion}

Food structure allows foods to be tailored controlling the extent and rate of lipid digestion. Potential applications for these foods include treatment of diet-related diseases and hunger suppression, bioactive delivery systems, or increased absorption of beneficial lipids. Factors to consider when formulating emulsion-based food products to modify lipid digestion, include emulsion droplet size, emulsifier type, oil type, and emulsion stability along the digestive tract. Development of delivery systems for functional ingredients must be able to deliver the functional component to its target site without sacrificing sensory properties or bioactive function. It is imperative to note that real food matrices are more complex than model conventional $\mathrm{o} / \mathrm{w}$ emulsions commonly used in in vitro studies. Therefore, future work must involve validating in vitro findings with human trials using real food matrices.

\subsection{References}

Abrams CK, Hamosh M, Dutta SK, et al (1987) Role of nonpancreatic lipolytic activity in exocrine pancreatic insufficiency. Gastroenterology 92:125-129 . doi: 10.1016/00165085(87)90848-1

Akhtar M, Murray BS, Afeisume EI, Khew SH (2014) Encapsulation of flavonoid in multiple emulsion using spinning disc reactor technology. Food Hydrocoll 34:62-67 . doi: 10.1016/j.foodhyd.2012.12.025

Armand M, Borel P, Dubois C, et al (1994) Characterization of emulsions and lipolysis of dietary lipids in the human stomach. Am J Physiol 266:G372-G381

Armand M, Borel P, Pasquier B, et al (1996) Physicochemical characteristics of emulsions during fat digestion in human stomach and duodenum. Am J Physiol Liver Physiol 271:G172-G183 . doi: 10.1152/ajpgi.1996.271.1.G172

Armand M, Borel P, Ythier P, et al (1992) Effects of droplet size, triacylglycerol composition, and calcium on the hydrolysis of complex emulsions by pancreatic lipase: an in vitro study. J Nutr Biochem. doi: 10.1016/0955-2863(92)90024-D

Bauer E, Jakob S, Mosenthin R (2005) Principles of physiology of lipid digestion. AsianAustralasian J Anim Sci 18:282-295 . doi: 10.5713/ajas.2005.282

Benichou A, Garti N (2004) Recent Developments in Double Emulsions for Food Applications. In: Food Emulsions, 4th ed. pp 353-412

Benichou A, Garti N (2010) Double Emulsions for Controlled-release Applications- Progress and Trends. In: Encyclopedic Handbook of Emulsion Technology. pp 377-407

Beysseriat M, Decker EA, McClements DJ (2006) Preliminary study of the influence of dietary fiber on the properties of oil-in-water emulsions passing through an in vitro human 
digestion model. Food Hydrocoll 20:800-809 . doi: 10.1016/j.foodhyd.2005.08.001

Boden G (2008) Obesity and Free Fatty Acids. Endocrinol. Metab. Clin. North Am. 37:635-646

Bonnaire L, Sandra S, Helgason T, et al (2008) Influence of lipid physical state on the in vitro digestibility of emulsified lipids. J Agric Food Chem 56:3791-3797 . doi: 10.1021/jf800159e

Borel P, Peyrot J, Portugal H, et al (2018) Digestion and absorption of 2 fat emulsions with different droplet sizes in the human digestive tract. Am J Clin Nutr 70:1096-1106 . doi: 10.1093/ajcn/70.6.1096

Bouwmeester H, Dekkers S, Noordam MY, et al (2009) Review of health safety aspects of nanotechnologies in food production. Regul Toxicol Pharmacol 53:52-62 . doi: 10.1016/J.YRTPH.2008.10.008

Bracco U (1994) Effect of triglyceride structure on fat absorption. In: American Journal of Clinical Nutrition

Bueschelberger HG, Tirok S, Stoffels I, Schoeppe A (2015) Lecithins. In: Emulsifiers in Food Technology: Second Edition. pp 21-60

Burey P, Bhandari BR, Howes T, Gidley MJ (2008) Hydrocolloid gel particles: Formation, characterization, and application. Crit Rev Food Sci Nutr 48:361-377 . doi: 10.1080/10408390701347801

Carey MC, Small DM, Bliss CM (1983) Lipid Digestion and Absorption. Annu Rev Physiol 45:651-677 . doi: 10.1146/annurev.ph.45.030183.003251

Carlson HC, Code CF, Nelson RA (1966) Motor action of the canine gastroduodenal junction: a cineradiographic, pressure, and electric study. Am J Dig Dis 11:155-72

Carriere F, Barrowman JA, Verger R, René L (1993) Secretion and contribution to lipolysis of gastric and pancreatic lipases during a test meal in humans. Gastroenterology 105:876-888 . doi: 10.1016/0016-5085(93)90908-U

Chang Y, McClements DJ (2016) Influence of emulsifier type on the in vitro digestion of fish oil-in-water emulsions in the presence of an anionic marine polysaccharide (fucoidan): Caseinate, whey protein, lecithin, or Tween 80. Food Hydrocoll 61:92-101 . doi: 10.1016/j.foodhyd.2016.04.047

Chaudhry Q, Scotter M, Blackburn J, et al (2008) Applications and implications of nanotechnologies for the food sector. Food Addit Contam Part A 25:241-258 . doi: $10.1080 / 02652030701744538$

Chen J, Zheng J, Decker EA, et al (2015) Improving nutraceutical bioavailability using mixed colloidal delivery systems: lipid nanoparticles increase tangeretin bioaccessibility and absorption from tangeretin-loaded zein nanoparticles. RSC Adv 5:73892-73900 . doi: $10.1039 / \mathrm{c} 5 \mathrm{ra} 13503 \mathrm{f}$

Chu BS, Gunning AP, Rich GT, et al (2010) Adsorption of bile salts and pancreatic colipase and lipase onto digalactosyldiacylglycerol and dipalmitoylphosphatidylcholine monolayers. Langmuir 26:9782-9793 . doi: 10.1021/la1000446

Cummings DE, Overduin J, Cummings DE, Overduin J (2007) Gastrointestinal regulation of food intake Find the latest version : Review series Gastrointestinal regulation of food intake. J Clin Invest 117:13-23 . doi: 10.1172/JCI30227.example

Dalgleish DG (2002) Conformations and structures of milk proteins adsorbed to oil-water interfaces. Food Res Int 29:541-547 . doi: 10.1016/s0963-9969(96)00065-8

Dalgleish DG (2004) Food Emulsions: Their Structures and Properties. In: Food Emulsions Day JPR, Rago G, Domke KF, et al (2010) Label-free imaging of lipophilic bioactive molecules 
during lipid digestion by multiplex coherent anti-stokes raman scattering microspectroscopy. J Am Chem Soc 132:8433-8439 . doi: 10.1021/ja102069d

De Oliveira SC, Bellanger A, Menard O, et al (2017) Impact of human milk pasteurization on gastric digestion in preterm infants: A randomized controlled trial. Am J Clin Nutr 105:379-390 . doi: 10.3945/ajcn.116.142539

de Oliveira SC, Deglaire A, Menard O, et al (2016) Holder pasteurization impacts the proteolysis, lipolysis and disintegration of human milk under in vitro dynamic term newborn digestion. Food Res Int 88:263-275 . doi: 10.1016/j.foodres.2015.11.022

Decher G (1997) Fuzzy Nanoassemblies: Toward Layered Polymeric Multicomposites. Science (80- ) 277:1232-1237 . doi: 10.1126/science.277.5330.1232

Decher G, Schlenoff JB (2012) Multilayer Thin Films : Sequential Assembly of Nanocomposite Materials. Wiley

DeNigris SJ, Hamosh M, Kasbekar DK, et al (1988) Lingual and gastric lipases: species differences in the origin of prepancreatic digestive lipases and in the localization of gastric lipase. Biochim Biophys Acta (BBA)/Lipids Lipid Metab 959:38-45 . doi: 10.1016/00052760(88)90147-6

Devraj R, Warren DB, Mullertz A, et al (2012) In vitro digestion testing of lipid-based delivery systems: Calcium ions combine with fatty acids liberated from triglyceride rich lipid solutions to form soaps and reduce the solubilization capacity of colloidal digestion products. Int J Pharm 441:323-333 . doi: 10.1016/j.ijpharm.2012.11.024

Dickinson E (2009) Hydrocolloids and emulsion stability. In: Handbook of Hydrocolloids: Second Edition

Dickinson E (2003) Hydrocolloids at interfaces and the influence on the properties of dispersed systems. Food Hydrocoll 17:25-39 . doi: 10.1016/S0268-005X(01)00120-5

Dickinson E (2010) Flocculation of protein-stabilized oil-in-water emulsions. Colloids Surfaces B Biointerfaces 81:130-140 . doi: 10.1016/j.colsurfb.2010.06.033

Dickinson E (1994) Protein-stabilized emulsions. J Food Eng 22:59-74 . doi: 10.1016/02608774(94)90025-6

Dickinson E (2011) Simple statistical thermodynamic model of the heteroaggregation and gelation of dispersions and emulsions. J Colloid Interface Sci 356:196-202 . doi: 10.1016/j.jcis.2011.01.036

Dickinson E (2017) Biopolymer-based particles as stabilizing agents for emulsions and foams. Food Hydrocoll 68:219-231 . doi: 10.1016/j.foodhyd.2016.06.024

Dickinson E (2015) Structuring of colloidal particles at interfaces and the relationship to food emulsion and foam stability. J Colloid Interface Sci 449:38-45 . doi:

10.1016/j.jcis.2014.09.080

Dickinson E, McClements DJ (1995) Advances in Food Colloids. Blackie Academic \& Professional

Dos Santos N, Cox KA, McKenzie CA, et al (2004) pH gradient loading of anthracyclines into cholesterol-free liposomes: enhancing drug loading rates through use of ethanol. Biochim Biophys Acta - Biomembr 1661:47-60 . doi: 10.1016/J.BBAMEM.2003.11.016

Dreher KD, Schulman JH, Hofmann AF (1967) Surface chemistry of the monoglyceride-bile salt system: Its relationship to the function of bile salts in fat absorption. J Colloid Interface Sci 25:71-83 . doi: 10.1016/0021-9797(67)90011-2

Ezhilarasi PN, Karthik P, Chhanwal N, Anandharamakrishnan C (2013) Nanoencapsulation Techniques for Food Bioactive Components: A Review. Food Bioprocess Technol 6:628- 
647 . doi: 10.1007/s11947-012-0944-0

Fang X, Rioux LE, Labrie S, Turgeon SL (2016) Disintegration and nutrients release from cheese with different textural properties during in vitro digestion. Food Res Int 88:276-283 . doi: 10.1016/j.foodres.2016.04.008

Favé G, Coste TC, Armand M (2004) Physicochemical properties of lipids: new strategies to manage fatty acid bioavailability. Cell Mol Biol (Noisy-le-grand) 50:815-31

Flanagan J, Singh H (2006) Microemulsions: A potential delivery system for bioactives in food. Crit Rev Food Sci Nutr 46:221-237 . doi: 10.1080/10408690590956710

Fritze A, Hens F, Kimpfler A, et al (2006) Remote loading of doxorubicin into liposomes driven by a transmembrane phosphate gradient. Biochim Biophys Acta - Biomembr 1758:16331640 . doi: 10.1016/J.BBAMEM.2006.05.028

Gamon BL, Virden JW, Berg JC (1989) The aggregation kinetics of an electrostatically stabilized dipalmitoyl phosphatidylcholine vesicle system. J Colloid Interface Sci 132:125138 . doi: 10.1016/0021-9797(89)90223-3

Giang TM, Gaucel S, Brestaz P, et al (2016) Dynamic modeling of in vitro lipid digestion: Individual fatty acid release and bioaccessibility kinetics. Food Chem 194:1180-1188 . doi: 10.1016/j.foodchem.2015.08.125

Golding M, Wooster TJ (2010) The influence of emulsion structure and stability on lipid digestion. Curr Opin Colloid Interface Sci 15:90-101 . doi: 10.1016/j.cocis.2009.11.006

Golding M, Wooster TJ, Day L, et al (2011) Impact of gastric structuring on the lipolysis of emulsified lipids. Soft Matter 7:3513 . doi: 10.1039/c0sm01227k

Goodman HM (2012) Hormones of the Gastrointestinal Tract. In: Basic Medical Endocrinology. Academic Press, pp 101-127

Grundy MML, Carrière F, Mackie AR, et al (2016a) The role of plant cell wall encapsulation and porosity in regulating lipolysis during the digestion of almond seeds. Food Funct 7:6978 . doi: 10.1039/C5FO00758E

Grundy MML, Grassby T, Mandalari G, et al (2015) Effect of mastication on lipid bioaccessibility of almonds in a randomized human study and its implications for digestion kinetics, metabolizable energy, and postprandial lipemia. Am J Clin Nutr 101:25-33 . doi: 10.3945/ajen.114.088328

Grundy MML, Lapsley K, Ellis PR (2016b) A review of the impact of processing on nutrient bioaccessibility and digestion of almonds. Int J Food Sci Technol 51:1937-1946 . doi: 10.1111/ijfs. 13192

Gu YS, Decker AE, McClements DJ (2005) Production and characterization of oil-in-water emulsions containing droplets stabilized by multilayer membranes consisting of $\beta$ lactoglobulin, 1-carrageenan and gelatin. Langmuir 21:5752-5760 . doi: 10.1021/la046888c

Guillot S, Moitzi C, Salentinig S, et al (2006) Direct and indirect thermal transitions from hexosomes to emulsified micro-emulsions in oil-loaded monoglyceride-based particles. Colloids Surfaces A Physicochem Eng Asp 291:78-84 . doi: 10.1016/j.colsurfa.2006.07.059

Guiotto EN, Cabezas DM, Diehl BWK, Tomás MC (2013) Characterization and emulsifying properties of different sunflower phosphatidylcholine enriched fractions. Eur J Lipid Sci Technol 115:865-873 . doi: 10.1002/ejlt.201200394

Gunning PA, Mackie AR, Gunning AP, et al (2004) Effect of surfactant type on surfactantprotein interactions at the air-water interface. Biomacromolecules 5:984-991 . doi: 10.1021/bm0344957

Guo Q, Ye A, Bellissimo N, et al (2017) Modulating fat digestion through food structure design. 
Prog Lipid Res 68:109-118 . doi: 10.1016/j.plipres.2017.10.001

Guri A, Gülseren I, Corredig M (2013) Utilization of solid lipid nanoparticles for enhanced delivery of curcumin in cocultures of HT29-MTX and Caco-2 cells. Food Funct 4:1410 1419 . doi: 10.1039/c3fo60180c

Guzey D, McClements DJ (2006) Formation, stability and properties of multilayer emulsions for application in the food industry. Adv Colloid Interface Sci 128-130:227-248 . doi: 10.1016/j.cis.2006.11.021

Guzey D, McClements DJ (2007) Impact of electrostatic interactions on formation and stability of emulsions containing oil droplets coated by $\beta$-lactoglobulin-pectin complexes. J Agric Food Chem 55:475-485 . doi: 10.1021/jf062342f

Hagens WI, Oomen AG, de Jong WH, et al (2007) What do we (need to) know about the kinetic properties of nanoparticles in the body? Regul Toxicol Pharmacol 49:217-229 . doi: 10.1016/J.YRTPH.2007.07.006

Hamosh M (1990a) Lingual and gastric lipases. Nutrition 6:421-8

Hamosh M (1990b) Lingual and gastric lipases : their role in fat digestion. CRC Press

Hamosh M, Klaeveman HL, Wolf RO, Scow RO (1975) Pharyngeal lipase and digestion of dietary triglyceride in man. J Clin Invest 55:908-913 . doi: 10.1172/JCI108019

Hasenhuettl GL (2008) Overview of Food Emulsifiers. In: Food Emulsifiers and Their Applications. Springer New York, New York, NY, pp 1-9

Hoffmann H, Reger M (2014) Emulsions with unique properties from proteins as emulsifiers. Adv Colloid Interface Sci 205:94-104 . doi: 10.1016/j.cis.2013.08.007

Hsu JP, Nacu A (2003) Behavior of soybean oil-in-water emulsion stabilized by nonionic surfactant. J Colloid Interface Sci 259:374-381 . doi: 10.1016/S0021-9797(02)00207-2

Hu L, Jia Y, Niu F, et al (2012) Preparation and enhancement of oral bioavailability of curcumin using microemulsions vehicle. J Agric Food Chem 60:7137-7141 . doi: 10.1021/jf204078t

$\mathrm{Hu}$ M, Li Y, Decker EA, McClements DJ (2010) Role of calcium and calcium-binding agents on the lipase digestibility of emulsified lipids using an in vitro digestion model. Food Hydrocoll 24:719-725 . doi: 10.1016/j.foodhyd.2010.03.010

Hur SJ, Decker EA, McClements DJ (2009) Influence of initial emulsifier type on microstructural changes occurring in emulsified lipids during in vitro digestion. Food Chem 114:253-262 . doi: 10.1016/j.foodchem.2008.09.069

Isailović BD, Kostić IT, Zvonar A, et al (2013) Resveratrol loaded liposomes produced by different techniques. Innov Food Sci Emerg Technol 19:181-189 . doi:

10.1016/J.IFSET.2013.03.006

Israelachvili JN (2011) Intermolecular and Surface Forces: Third Edition

Jani P, Halbert GW, Langridge J, Florence AT (1990) Nanoparticle uptake by the rat gastrointestinal mucosa: quantitation and particle size dependency. J Pharm Pharmacol 42:821-6

Jódar-Reyes AB, Torcello-Gómez A, Wulff-Pérez M, et al (2010) Different stability regimes of oil-in-water emulsions in the presence of bile salts. Food Res Int 43:1634-1641 . doi: 10.1016/j.foodres.2010.05.005

Joye IJ, Davidov-Pardo G, McClements DJ (2014) Nanotechnology for increased micronutrient bioavailability. Trends Food Sci Technol 40:168-182 . doi: 10.1016/j.tifs.2014.08.006

Kalantzi L, Goumas K, Kalioras V, et al (2006) Characterization of the Human Upper Gastrointestinal Contents Under Conditions Simulating Bioavailability/Bioequivalence Studies. Pharm Res 23:165-176 . doi: 10.1007/s11095-005-8476-1 
Karasov WH (2017) Integrative physiology of transcellular and paracellular intestinal absorption. J Exp Biol 220:2495-2501 . doi: 10.1242/jeb.144048

Kargar M, Fayazmanesh K, Alavi M, et al (2012) Investigation into the potential ability of Pickering emulsions (food-grade particles) to enhance the oxidative stability of oil-in-water emulsions. J Colloid Interface Sci 366:209-215 . doi: 10.1016/J.JCIS.2011.09.073

Karupaiah T, Sundram K (2007) Effects of stereospecific positioning of fatty acids in triacylglycerol structures in native and randomized fats: A review of their nutritional implications. Nutr Metab 4:16 . doi: 10.1186/1743-7075-4-16

Keowmaneechai E, McClements DJ (2006) Effect of CaCl2 and $\mathrm{KCl}$ on Physiochemical Properties of Model Nutritional Beverages Based on Whey Protein Stabilized Oil-in-Water Emulsions. J Food Sci 67:665-671 . doi: 10.1111/j.1365-2621.2002.tb10657.x

Kralova I, Sjöblom J (2009) Surfactants used in food industry: A review. J Dispers Sci Technol 30:1363-1383 . doi: 10.1080/01932690902735561

Lam RSH, Nickerson MT (2013) Food proteins: A review on their emulsifying properties using a structure-function approach. Food Chem 141:975-984 . doi:

10.1016/j.foodchem.2013.04.038

Lamothe S, Rémillard N, Tremblay J, Britten M (2017) Influence of dairy matrices on nutrient release in a simulated gastrointestinal environment. Food Res Int 92:138-146 . doi: 10.1016/j.foodres.2016.12.026

Lee SJ, Choi SJ, Li Y, et al (2011) Protein-stabilized nanoemulsions and emulsions: Comparison of physicochemical stability, lipid oxidation, and lipase digestibility. J Agric Food Chem 59:415-427 . doi: 10.1021/jf103511v

Levine AS, Silvis SE (2010) Absorption of Whole Peanuts, Peanut Oil, and Peanut Butter. N Engl J Med 303:917-918 . doi: 10.1056/nejm198010163031605

Li J, Ye A, Lee SJ, Singh H (2012) Influence of gastric digestive reaction on subsequent in vitro intestinal digestion of sodium caseinate-stabilized emulsions. Food Funct 3:320-326 . doi: $10.1039 / \mathrm{c} 2$ fo10242k

Li Y, Hu M, McClements DJ (2011) Factors affecting lipase digestibility of emulsified lipids using an in vitro digestion model: Proposal for a standardised $\mathrm{pH}$-stat method. Food Chem 126:498-505 . doi: 10.1016/j.foodchem.2010.11.027

Li Y, Mcclements DJ (2010) New mathematical model for interpreting ph-stat digestion profiles: Impact of lipid droplet characteristics on in vitro digestibility. J Agric Food Chem 58:80858092 . doi: 10.1021/jf101325m

Liang R, Jiang Y, Yokoyama W, et al (2016) Preparation of Pickering emulsions with short, medium and long chain triacylglycerols stabilized by starch nanocrystals and their: In vitro digestion properties. RSC Adv 6:99496-99508 . doi: 10.1039/c6ra18468e

Lieverse RJ, Masclee AAM, Jansen JBMJ, et al (1995) Satiety effects of the type A CCK receptor antagonist loxiglumide in lean and obese women. Biol Psychiatry 37:331-335 . doi: 10.1016/0006-3223(94)00136-Q

Lopez C (2011) Milk fat globules enveloped by their biological membrane: Unique colloidal assemblies with a specific composition and structure. Curr. Opin. Colloid Interface Sci. $16: 391-404$

Lundin L, Golding M (2009) Structure design for healthy food. Aust J Dairy Technol 64:68-75

Ma W, Tang C, Lai L (2005) Specificity of trypsin and chymotrypsin: Loop-motion-controlled dynamic correlation as a determinant. Biophys J 89:1183-1193 . doi:

10.1529/biophysj.104.057158 
Macierzanka A, Sancho AI, Mills ENC, et al (2009) Emulsification alters simulated gastrointestinal proteolysis of $\beta$-casein and $\beta$-lactoglobulin. Soft Matter 5:538-550 . doi: 10.1039/B811233A

Mackie A, Macierzanka A (2010) Colloidal aspects of protein digestion. Curr Opin Colloid Interface Sci 15:102-108 . doi: 10.1016/j.cocis.2009.11.005

Mackie AR, Gunning AP, Wilde PJ, Morris VJ (2000) Orogenic displacement of protein from the oil/water interface. Langmuir 16:2242-2247 . doi: 10.1021/1a990711e

Madadlou A, Rakhshi E, Abbaspourrad A (2016) Engineered emulsions for obesity treatment. Trends Food Sci Technol 52:90-97 . doi: 10.1016/j.tifs.2016.04.009

Malaki Nik A, Wright AJ, Corredig M (2010) Surface adsorption alters the susceptibility of whey proteins to pepsin-digestion. J Colloid Interface Sci 344:372-381 . doi: 10.1016/j.jcis.2010.01.006

Maldonado-Valderrama J, Gunning AP, Wilde PJ, Morris VJ (2010) In vitro gastric digestion of interfacial protein structures: Visualisation by AFM. Soft Matter 6:4908-4915 . doi: 10.1039/c0sm00300j

Maldonado-Valderrama J, Wilde P, MacIerzanka A, MacKie A (2011) The role of bile salts in digestion. Adv Colloid Interface Sci 165:36-46 . doi: 10.1016/j.cis.2010.12.002

Maldonado-Valderrama J, Woodward NC, Patrick Gunning A, et al (2008) Interfacial characterization of $\beta$-lactoglobulin networks: Displacement by bile salts. Langmuir 24:6759-6767 . doi: 10.1021/la800551u

Maljaars PWJ, Peters HPF, Mela DJ, Masclee AAM (2008a) Ileal brake: A sensible food target for appetite control. A review. Physiol Behav 95:271-281 . doi: 10.1016/j.physbeh.2008.07.018

Maljaars PWJ, Symersky T, Kee BC, et al (2008b) Effect of ileal fat perfusion on satiety and hormone release in healthy volunteers. Int J Obes. doi: 10.1038/ijo.2008.166

Mantovani RA, Cavallieri ÂLF, Netto FM, Cunha RL (2013) Stability and in vitro digestibility of emulsions containing lecithin and whey proteins. Food Funct 4:1322-1331 . doi: 10.1039/c3fo60156k

Mao Y, McClements DJ (2011) Modulation of bulk physicochemical properties of emulsions by hetero-aggregation of oppositely charged protein-coated lipid droplets. Food Hydrocoll 25:1201-1209 . doi: 10.1016/j.foodhyd.2010.11.007

Marciani L, Wickham M, Singh G, et al (2007) Enhancement of intragastric acid stability of a fat emulsion meal delays gastric emptying and increases cholecystokinin release and gallbladder contraction. Am J Physiol Liver Physiol 292:G1607-G1613 . doi: 10.1152/ajpgi.00452.2006

Mason TG, Wilking JN, Meleson K, et al (2006) Nanoemulsions: formation, structure, and physical properties. J Phys Condens Matter 18:R635-R666 . doi: 10.1088/09538984/18/41/R01

Matalanis A, Jones OG, McClements DJ (2011) Structured biopolymer-based delivery systems for encapsulation, protection, and release of lipophilic compounds. Food Hydrocoll 25:1865-1880 . doi: 10.1016/j.foodhyd.2011.04.014

McClements DJ (2018) The biophysics of digestion: lipids. Curr Opin Food Sci 21:1-6 . doi: 10.1016/j.cofs.2018.03.009

McClements DJ (2013) Edible lipid nanoparticles: Digestion, absorption, and potential toxicity. Prog Lipid Res 52:409-423 . doi: 10.1016/j.plipres.2013.04.008

McClements DJ (2004a) Food Emulsions: Principles, Practices, and Techniques, Second Edition 
McClements DJ (2004b) Protein-stabilized emulsions. Curr. Opin. Colloid Interface Sci. 9:305313

McClements DJ (2014) Nanoparticle- and microparticle-based delivery systems: Encapsulation, protection and release of active compounds

McClements DJ (2012) Advances in fabrication of emulsions with enhanced functionality using structural design principles. Curr Opin Colloid Interface Sci 17:235-245 . doi:

10.1016/j.cocis.2012.06.002

McClements DJ (2017) The future of food colloids: Next-generation nanoparticle delivery systems. Curr Opin Colloid Interface Sci 28:7-14 . doi: 10.1016/j.cocis.2016.12.002

McClements DJ, Decker EA, Park Y, Weiss J (2008) Designing food structure to control stability, digestion, release and absorption of lipophilic food components. Food Biophys 3:219-228 . doi: 10.1007/s11483-008-9070-y

McClements DJ, Decker EA, Weiss J (2007) Emulsion-Based Delivery Systems for Lipophilic Bioactive Components. J Food Sci 72:R109-R124 . doi: 10.1111/j.1750-3841.2007.00507.x

McClements DJ, Gumus CE (2016) Natural emulsifiers - Biosurfactants, phospholipids, biopolymers, and colloidal particles: Molecular and physicochemical basis of functional performance. Adv Colloid Interface Sci 234:3-26 . doi: 10.1016/j.cis.2016.03.002

McClements DJ, Li Y (2010) Structured emulsion-based delivery systems: Controlling the digestion and release of lipophilic food components. Adv Colloid Interface Sci 159:213228 . doi: 10.1016/j.cis.2010.06.010

McClements DJ, Monahan FJ, Kinsella JE (1993) Disulfide Bond Formation Affects Stability of Whey Protein Isolate Emulsions. J Food Sci 58:1036-1039 . doi: 10.1111/j.13652621.1993.tb06106.x

Meynier A, Genot C (2017) Molecular and structural organization of lipids in foods: their fate during digestion and impact in nutrition. OCL 24:D202 . doi: 10.1051/oc1/2017006

Monahan FJ, McClements DJ, Kinsella JE (1993) Polymerization of Whey Proteins in Whey Protein-Stabilized Emulsions. J Agric Food Chem 41:1826-1829 . doi: 10.1021/jf00035a004

Moreau H, Laugier R, Gargouri Y, et al (1988) Human preduodenal lipase is entirely of gastric fundic origin. Gastroenterology 95:1221-1226 . doi: 10.1016/0016-5085(88)90354-X

Moschakis T, Murray BS, Biliaderis CG (2010) Modifications in stability and structure of whey protein-coated $\mathrm{o} / \mathrm{w}$ emulsions by interacting chitosan and gum arabic mixed dispersions. Food Hydrocoll 24:8-17 . doi: 10.1016/J.FOODHYD.2009.07.001

Mu H, Høy CE (2004) The digestion of dietary triacylglycerols. Prog Lipid Res 43:105-133 . doi: 10.1016/S0163-7827(03)00050-X

$\mathrm{Mu}$ H, Porsgaard T (2005) The metabolism of structured triacylglycerols. Prog. Lipid Res. 44:430-448

Mulet-Cabero AI, Rigby NM, Brodkorb A, Mackie AR (2017) Dairy food structures influence the rates of nutrient digestion through different in vitro gastric behaviour. Food Hydrocoll 67:63-73 . doi: 10.1016/j.foodhyd.2016.12.039

Mun S, Decker EA, McClements DJ (2007) Influence of emulsifier type on in vitro digestibility of lipid droplets by pancreatic lipase. Food Res Int 40:770-781 . doi:

10.1016/j.foodres.2007.01.007

Mun S, Decker EA, McClements DJ (2005) Influence of droplet characteristics on the formation of oil-in-water emulsions stabilized by surfactant-chitosan layers. Langmuir 21:6228-6234 . doi: $10.1021 / \mathrm{la} 050502 \mathrm{w}$ 
Mun S, Decker EA, Park Y, et al (2006) Influence of interfacial composition on in vitro digestibility of emulsified lipids: Potential mechanism for chitosan's ability to inhibit fat digestion. Food Biophys 1:21-29 . doi: 10.1007/s11483-005-9001-0

Nelen BAP, Bax L, Cooper JM (2015) Sucrose Esters. In: Emulsifiers in Food Technology: Second Edition

Nyemb K, Guérin-Dubiard C, Dupont D, et al (2014) The extent of ovalbumin in vitro digestion and the nature of generated peptides are modulated by the morphology of protein aggregates. Food Chem 157:429-438 . doi: 10.1016/J.FOODCHEM.2014.02.048

Nyemb K, Guérin-Dubiard C, Pézennec S, et al (2016) The structural properties of egg white gels impact the extent of in vitro protein digestion and the nature of peptides generated. Food Hydrocoll 54:315-327 . doi: 10.1016/J.FOODHYD.2015.10.011

O'Callaghan Y, O’Brien N (2010) Bioaccessibility, cellular uptake and transepithelial transport of ??-tocopherol and retinol from a range of supplemented foodstuffs assessed using the caco-2 cell model. Int J Food Sci Technol 45:1436-1442 . doi: 10.1111/j.13652621.2010.02285.x

Olsen J V., Ong S-E, Mann M (2004) Trypsin Cleaves Exclusively C-terminal to Arginine and Lysine Residues. Mol Cell Proteomics 3:608-614 . doi: 10.1074/mcp.t400003-mcp200

Ozturk B, Argin S, Ozilgen M, McClements DJ (2014) Formation and stabilization of nanoemulsion-based vitamin e delivery systems using natural surfactants: Quillaja saponin and lecithin. J Food Eng 142:57-63 . doi: 10.1016/j.jfoodeng.2014.06.015

Ozturk B, Argin S, Ozilgen M, McClements DJ (2015) Formation and stabilization of nanoemulsion-based vitamin E delivery systems using natural biopolymers: Whey protein isolate and gum arabic. Food Chem 188:256-263 . doi: 10.1016/j.foodchem.2015.05.005

Pafumi Y, Lairon D, De La Porte PL, et al (2002) Mechanisms of inhibition of triacylglycerol hydrolysis by human gastric lipase. J Biol Chem. doi: 10.1074/jbc.M202839200

Park HJ (1999) Development of advanced edible coatings for fruits. Trends Food Sci Technol 10:254-260 . doi: 10.1016/S0924-2244(00)00003-0

Parrish CR (2017) The Use of Medium-Chain Triglycerides in Gastrointestinal Disorders. Nutr Issues Gastroenterol

Patel G (2011) Oral Delivery of Proteins and Peptides: Concepts and Applications. In: Challenges in Delivery of Therapeutic Genomics and Proteomics. Elsevier, pp 481-529

Patra D, Sanyal A, Rotello VM (2010) Colloidal Microcapsules: Self-Assembly of Nanoparticles at the Liquid-Liquid Interface. Chem - An Asian J 5:2442-2453 . doi: 10.1002/asia.201000301

Patton JS, Carey MC (1979) Watching fat digestion. Science 204:145-8 . doi: 10.1126/SCIENCE.432636

Pich AZ, Adler HJP (2007) Composite aqueous microgels: An overview of recent advances in synthesis, characterization and application. Polym Int 56:291-307 . doi: 10.1002/pi.2142

Pinheiro AC, Lad M, Silva HD, et al (2013) Unravelling the behaviour of curcumin nanoemulsions during in vitro digestion: Effect of the surface charge. Soft Matter 9:31473154 . doi: $10.1039 / \mathrm{c} 3 \mathrm{sm} 27527 \mathrm{~b}$

Pond SM, Tozer TN (1984) First-Pass Elimination Basic Concepts and Clinical Consequences. Clin Pharmacokinet 9:1-25 . doi: 10.2165/00003088-198409010-00001

Pool H, Mendoza S, Xiao H, McClements DJ (2013) Encapsulation and release of hydrophobic bioactive components in nanoemulsion-based delivery systems: Impact of physical form on quercetin bioaccessibility. Food Funct 4:162-174 . doi: 10.1039/c2fo30042g 
Qian C, Decker EA, Xiao H, McClements DJ (2012) Nanoemulsion delivery systems: Influence of carrier oil on $\beta$-carotene bioaccessibility. Food Chem 135:1440-1447 . doi: 10.1016/j.foodchem.2012.06.047

Ramírez M, Amate L, Gil A (2001) Absorption and distribution of dietary fatty acids from different sources. Early Hum Dev 65:S95-S101 . doi: 10.1016/S0378-3782(01)00211-0

Rao J, McClements DJ (2011) Food-grade microemulsions, nanoemulsions and emulsions: Fabrication from sucrose monopalmitate \& lemon oil. Food Hydrocoll 25:1413-1423 . doi: 10.1016/j.foodhyd.2011.02.004

Raynal-Ljutovac K, Bouvier J, Gayet C, et al (2011) Organisation structurale et moléculaire des lipides dans les aliments : impacts possibles sur leur digestion et leur assimilation par l'Homme. OCL - Ol Corps Gras Lipides 18:324-351 . doi: 10.1684/ocl.2011.0415

Rayner M, Marku D, Eriksson M, et al (2014) Biomass-based particles for the formulation of Pickering type emulsions in food and topical applications. Colloids Surfaces A Physicochem Eng Asp 458:48-62 . doi: 10.1016/J.COLSURFA.2014.03.053

Reboul E, Richelle M, Perrot E, et al (2006) Bioaccessibility of carotenoids and vitamin E from their main dietary sources. J Agric Food Chem 54:8749-8755 . doi: 10.1021/jf061818s

Reis PM, Raab TW, Chuat JY, et al (2008) Influence of surfactants on lipase fat digestion in a model gastro-intestinal system. Food Biophys 3:370-381 . doi: 10.1007/s11483-008-9091-6

Reza Mozafari M, Johnson C, Hatziantoniou S, Demetzos C (2008) Nanoliposomes and Their Applications in Food Nanotechnology. J Liposome Res 18:309-327 . doi: $10.1080 / 08982100802465941$

Rinderknecht H (2005) Activation of pancreatic zymogens. Dig Dis Sci 31:314-321 . doi: 10.1007/bf01318124

Sagalowicz L, Leser ME (2010) Delivery systems for liquid food products. Curr Opin Colloid Interface Sci 15:61-72 . doi: 10.1016/j.cocis.2009.12.003

Saito M, Kasai M, Watanabe S, et al (2003) Effects of Highly Purified Structured Lipids Containing Medium-chain Fatty Acids and Linoleic Acid on Lipid Profiles in Rats. Biosci Biotechnol Biochem 67:1937-1943 . doi: 10.1271/bbb.67.1937

Salentinig S, Sagalowicz L, Leser ME, et al (2011) Transitions in the internal structure of lipid droplets during fat digestion. Soft Matter 7:650-661 . doi: 10.1039/C0SM00491J

Salvia-Trujillo L, Qian C, Martín-Belloso O, McClements DJ (2013a) Influence of particle size on lipid digestion and $\beta$-carotene bioaccessibility in emulsions and nanoemulsions. Food Chem 141:1475-1480 . doi: 10.1016/j.foodchem.2013.03.050

Salvia-Trujillo L, Qian C, Martín-Belloso O, McClements DJ (2013b) Modulating $\beta$-carotene bioaccessibility by controlling oil composition and concentration in edible nanoemulsions. Food Chem 139:878-884 . doi: 10.1016/j.foodchem.2013.02.024

Salvia-Trujillo L, Verkempinck SHE, Sun L, et al (2017) Lipid digestion, micelle formation and carotenoid bioaccessibility kinetics: Influence of emulsion droplet size. Food Chem 229:653-662 . doi: 10.1016/j.foodchem.2017.02.146

Sarkar A, Goh KKT, Singh H (2009) Colloidal stability and interactions of milk-proteinstabilized emulsions in an artificial saliva. Food Hydrocoll 23:1270-1278 . doi: 10.1016/j.foodhyd.2008.09.008

Sarkar A, Murray B, Holmes M, et al (2016a) In vitro digestion of Pickering emulsions stabilized by soft whey protein microgel particles: influence of thermal treatment. Soft Matter 12:3558-3569 . doi: 10.1039/C5SM02998H

Sarkar A, Ye A, Singh H (2016b) On the role of bile salts in the digestion of emulsified lipids. 
Food Hydrocoll 60:77-84 . doi: 10.1016/j.foodhyd.2016.03.018

Schirra J, Göke B (2005) The physiological role of GLP-1 in human: Incretin, ileal brake or more? Regul. Pept.

Scow RO (1988) Effect of sodium taurodeoxycholate, $\mathrm{CaCl} 2$ and albumin on the action of pancreatic lipase on droplets of trioleoylglycerol and the release of lipolytic products into aqueous media. Biochimie 70:1251-1261 . doi: 10.1016/0300-9084(88)90192-7

Sek L, Porter CJH, Kaukonen AM, Charman WN (2002) Evaluation of the in-vitro digestion profiles of long and medium chain glycerides and the phase behaviour of their lipolytic products. J Pharm Pharmacol 54:29-41 . doi: 10.1211/0022357021771896

Sekhon BS (2010) Food nanotechnology - an overview. Nanotechnol Sci Appl 3:1-15

Sensoy I (2014) A Review on the Relationship Between Food Structure, Processing, and Bioavailability. Crit Rev Food Sci Nutr 54:902-909 . doi: 10.1080/10408398.2011.619016

Sessa M, Balestrieri ML, Ferrari G, et al (2014) Bioavailability of encapsulated resveratrol into nanoemulsion-based delivery systems. Food Chem 147:42-50 . doi: 10.1016/j.foodchem.2013.09.088

Shimoni G, Shani Levi C, Levi Tal S, Lesmes U (2013) Emulsions stabilization by lactoferrin nano-particles under invitro digestion conditions. Food Hydrocoll 33:264-272 . doi: 10.1016/j.foodhyd.2013.03.017

Singh H (2005) Protein functionality and aggregation. Milk protein functionality in food colloids. In: Food Colloids: Interactions, Microstructure and Processing. pp 177-193

Singh H, Sarkar A (2011) Behaviour of protein-stabilised emulsions under various physiological conditions. Adv Colloid Interface Sci 165:47-57 . doi: 10.1016/J.CIS.2011.02.001

Singh H, Thompson A, Liu W, Corredig M (2012) Liposomes as food ingredients and nutraceutical delivery systems. Encapsulation Technol Deliv Syst Food Ingredients Nutraceuticals 287-318 . doi: 10.1533/9780857095909.3.287

Singh H, Ye A (2013) Structural and biochemical factors affecting the digestion of proteinstabilized emulsions. Curr Opin Colloid Interface Sci 18:360-370 . doi: 10.1016/j.cocis.2013.04.006

Singh H, Ye A, Horne D (2009) Structuring food emulsions in the gastrointestinal tract to modify lipid digestion. Prog Lipid Res 48:92-100 . doi: 10.1016/j.plipres.2008.12.001

Souto EB, Martins-Lopes P, Lopes CM, et al (2009) A Note on Regulatory Concerns and Toxicity Assessment in Lipid-Based Delivery Systems (LDS). J Biomed Nanotechnol 5:317-322 . doi: 10.1166/jbn.2009.1041

Speranza A, Corradini MG, Hartman TG, et al (2013) Influence of emulsifier structure on lipid bioaccessibility in oil-water nanoemulsions. J Agric Food Chem 61:6505-6515 . doi: $10.1021 / \mathrm{jf} 401548 \mathrm{r}$

Spernath A, Aserin A (2006) Microemulsions as carriers for drugs and nutraceuticals. Adv Colloid Interface Sci 128-130:47-64 . doi: 10.1016/j.cis.2006.11.016

Stanghellini V, Ruggeri E, Go VLW, et al (2016) Fat-induced heal brake in humans: A dosedependent phenomenon correlated to the plasma levels of peptide YY. Gastroenterology 105:733-739 . doi: 10.1016/0016-5085(93)90890-o

Steingoetter A, Radovic T, Buetikofer S, et al (2015) Imaging gastric structuring of lipid emulsions and its effect on gastrointestinal function: A randomized trial in healthy subjects. Am J Clin Nutr 101:714-724 . doi: 10.3945/ajcn.114.100263

Stipanuk MH (2006) Biochemical, physiological, and molecular aspects of human nutrition, 2nd ed. Saunders Elsevier, Philadelphia, Pa Edinburgh 
Taylor TM, Weiss J, Davidson PM, Bruce BD (2005) Liposomal Nanocapsules in Food Science and Agriculture. Crit Rev Food Sci Nutr 45:587-605 . doi: 10.1080/10408390591001135

Tcholakova S, Denkov ND, Lips A (2008) Comparison of solid particles, globular proteins and surfactants as emulsifiers. Phys. Chem. Chem. Phys.

Thilakarathna SH, Rogers M, Lan Y, et al (2016) Investigations of in vitro bioaccessibility from interesterified stearic and oleic acid-rich blends. Food Funct 7:1932-1940 . doi: $10.1039 / \mathrm{c} 5$ fo0 $01272 \mathrm{~d}$

Torcello-Gómez A, Jódar-Reyes AB, Maldonado-Valderrama J, Martín-Rodríguez A (2012) Effect of emulsifier type against the action of bile salts at oil-water interfaces. Food Res Int 48:140-147 . doi: 10.1016/j.foodres.2012.03.007

Torcello-Gómez A, Maldonado-Valderrama J, Martín-Rodríguez A, McClements DJ (2011) Physicochemical properties and digestibility of emulsified lipids in simulated intestinal fluids: Influence of interfacial characteristics. Soft Matter 7:6167-6177 . doi: $10.1039 / \mathrm{c} 1 \mathrm{sm} 05322 \mathrm{a}$

Ubbink J, Burbidge A, Mezzenga R (2008) Food structure and functionality: A soft matter perspective. Soft Matter 4:1569-1581 . doi: 10.1039/b802183j

Vajda T, Szabo T (1976) Specificity of trypsin and alpha-chymotrypsin towards neutral substrates. Acta Biochim Biophys Acad Sci Hung 11:287-294

van Nieuwenhuyzen W, Szuhaj BF (2002) Effects of lecithins and proteins on the stability of emulsions. Lipid - Fett 100:282-291 . doi: 10.1002/(sici)15214133(199807)100:7<282::aid-lipi282>3.3.co;2-n

Verkempinck SHE, Salvia-Trujillo L, Moens LG, et al (2018) Emulsion stability during gastrointestinal conditions effects lipid digestion kinetics. Food Chem 246:179-191 . doi: 10.1016/j.foodchem.2017.11.001

Vingerhoeds MH, Blijdenstein TBJ, Zoet FD, Van Aken GA (2005) Emulsion flocculation induced by saliva and mucin. Food Hydrocoll 19:915-922 . doi: 10.1016/j.foodhyd.2004.12.005

Vingerhoeds MH, Silletti E, de Groot J, et al (2009) Relating the effect of saliva-induced emulsion flocculation on rheological properties and retention on the tongue surface with sensory perception. Food Hydrocoll 23:773-785 . doi: 10.1016/j.foodhyd.2008.04.014

Wahbeh GT, Christie DL (2011) Basic Aspects of Digestion and Absorption. In: Pediatric Gastrointestinal and Liver Disease. W.B. Saunders, p 10-19.e2

Weiss J, Decker EA, McClements DJ, et al (2008) Solid lipid nanoparticles as delivery systems for bioactive food components. Food Biophys 3:146-154 . doi: 10.1007/s11483-008-9065-8

Weiss J, Takhistov P, McClements DJ (2006) Functional materials in food nanotechnology. J Food Sci 71:R107-R116 . doi: 10.1111/j.1750-3841.2006.00195.x

Wickham M, Wilde P, Fillery-Travis A (2002) A physicochemical investigation of two phosphatidylcholine/bile salt interfaces: Implications for lipase activation. Biochim Biophys Acta - Mol Cell Biol Lipids. doi: 10.1016/S1388-1981(01)00196-2

Wierenga PA, Egmond MR, Voragen AGJ, de Jongh HHJ (2006) The adsorption and unfolding kinetics determines the folding state of proteins at the air-water interface and thereby the equation of state. J Colloid Interface Sci. doi: 10.1016/j.jcis.2006.03.016

Wilde P, Mackie A, Husband F, et al (2004) Proteins and emulsifiers at liquid interfaces. Adv Colloid Interface Sci 108-109:63-71 . doi: 10.1016/j.cis.2003.10.011

Wilde PJ, Chu BS (2011) Interfacial \& colloidal aspects of lipid digestion. Adv Colloid Interface Sci 165:14-22 . doi: 10.1016/j.cis.2011.02.004 
Xiang S, Yao X, Zhang W, et al (2015) Gum Arabic-stabilized conjugated linoleic acid emulsions: Emulsion properties in relation to interfacial adsorption behaviors. Food Hydrocoll 48:110-116 . doi: 10.1016/j.foodhyd.2015.01.033

Yang Y, McClements DJ (2013) Vitamin E bioaccessibility: Influence of carrier oil type on digestion and release of emulsified $\alpha$-tocopherol acetate. Food Chem 141:473-481 . doi: 10.1016/j.foodchem.2013.03.033

Yao M, McClements DJ, Xiao H (2015) Improving oral bioavailability of nutraceuticals by engineered nanoparticle-based delivery systems. Curr Opin Food Sci 2:14-19 . doi: 10.1016/j.cofs.2014.12.005

Ye A, Cui J, Zhu X, Singh H (2013) Effect of calcium on the kinetics of free fatty acid release during in vitro lipid digestion in model emulsions. Food Chem 139:681-688 . doi: 10.1016/j.foodchem.2013.02.014

Ythier P, Lairon D, Melin C, et al (2003) Hydrolysis of emulsions with different triglycerides and droplet sizes by gastric lipase in vitro. Effect on pancreatic lipase activity. J Nutr Biochem 5:124-133 . doi: 10.1016/0955-2863(94)90083-3

Zangenberg NH, Müllertz A, Kristensen HG, Hovgaard L (2001) A dynamic in vitro lipolysis model. I. Controlling the rate of lipolysis by continuous addition of calcium. Eur J Pharm Sci 14:115-122 . doi: 10.1016/S0928-0987(01)00169-5

Zhai J, Wooster TJ, Hoffmann S V., et al (2011) Structural rearrangement of $\beta$-lactoglobulin at different oil-water interfaces and its effect on emulsion stability. Langmuir. doi: $10.1021 / 1 a 201483 y$

Zhang R, McClements DJ (2016) Enhancing nutraceutical bioavailability by controlling the composition and structure of gastrointestinal contents: Emulsion-based delivery and excipient systems. Food Struct 10:21-36 . doi: 10.1016/j.foostr.2016.07.006

Zhang R, Zhang Z, Zhang H, et al (2015) Influence of emulsifier type on gastrointestinal fate of oil-in-water emulsions containing anionic dietary fiber (pectin). Food Hydrocoll 45:175185 . doi: 10.1016/j.foodhyd.2014.11.020

Zou L, Zheng B, Zhang R, et al (2016) Enhancing the bioaccessibility of hydrophobic bioactive agents using mixed colloidal dispersions: Curcumin-loaded zein nanoparticles plus digestible lipid nanoparticles. Food Res Int 81:74-82 . doi: 10.1016/j.foodres.2015.12.035

Zucker D, Marcus D, Barenholz Y, Goldblum A (2009) Liposome drugs' loading efficiency: A working model based on loading conditions and drug's physicochemical properties. J Control Release 139:73-80 . doi: 10.1016/J.JCONREL.2009.05.036 


\section{Chapter 3 Surfactants ${ }^{1}$}

\subsection{Introduction to Food Emulsions}

An emulsion consists of two liquid immiscible phases: oil and water. Either of these two phases can be the continuous or the dispersed phase and as such there are two primary types of emulsions: oil-in-water (o/w) or water-in-oil (w/o). An o/w emulsion consists of an oil phase dispersed into a continuous water (aqueous) phase. Common food examples include: mayonnaise, salad dressing, whipped toppings, and ice cream. On the contrary, a w/o emulsion consists of an aqueous phase dispersed in a continuous oil phase, such as butter and margarine (Kralova and Sjöblom 2009). In addition to an oil phase and an aqueous phase, the formation of an emulsion requires energy input and addition of surfactants. The resulting emulsion that is formed $(\mathrm{o} / \mathrm{w}$ or w/o) is greatly influenced by the chemistry of surfactant (Walstra 1993). Surfactants are used to reduce the work required to overcome surface free energy and disperse one phase into the other and to stabilize the final emulsion. Properties of o/w emulsions rely more heavily on the type of surfactant used and the composition of the aqueous phase; while, properties of w/o emulsions are more dependent on the type of fat (Kralova and Sjöblom 2009).

Food emulsions exist in a pseudo-equilibrium or 'frozen' kinetic state making them "shelfstable" only for a limited time. The stability of the emulsion is dependent on the crystallinity of oil/fat droplets, the interfacial material, and the chemistry of the aqueous phase. The degree of crystallinity of the oil phase influences its melting properties, which is important for partial coalescence in ice cream and whipped products. The quality of the interfacial layer depends on the mixture of macromolecules and small molecular weight (MW) surfactants. The quantity and

\footnotetext{
${ }^{1}$ Reprinted from Encyclopedia of Food Chemistry (Shahidi, F., Stadler, R., Sun-Waterhouse, D., Warelis, P.), $1^{\text {st }}$ edition, Surfactants, Pages 276-282, Ng, N., Rogers, M.A., Copyright (2019), with permission from Elsevier.
} 
structure of the adsorbed surface-active compounds affects interfacial properties. Small MW surfactants often displace adsorbed proteins or bind/interact with proteins (i.e., lecithin), which modifies emulsion droplet stability. Therefore, even small changes in interfacial composition can significantly influence overall stability. The interactions between emulsion droplets and the aqueous phase also impacts emulsion stability (Dalgleish 2006).

The terms emulsifier, surface-active agents and surfactant are often used interchangeably to describe amphiphilic compounds that contain both polar and non-polar residues. This characteristic allows them to reside at the oil-water interface and reduce the interfacial tension (Weiss 1971; Lauridsen 1976). This general review will focus on the mechanisms of emulsification, prevention of emulsion destabilization, common surfactants, and their applications in food.

\subsection{Mechanisms of Emulsification}

Emulsions are thermodynamically unstable because they have a positive free energy of formation $\left(\Delta \mathrm{G}_{\mathrm{f}}\right)$ and are considered non-equilibrium systems as emulsified droplet sizes increase with time (Bibette et al. 1992; Taylor 1998). This instability is due to the large interfacial area of droplets, represented by surface area (A) \& interfacial tension $(\gamma)$ :

$$
\Delta G_{f}=\gamma A-T \Delta S_{f}
$$

The entropy of formation $\left(\Delta \mathrm{S}_{\mathrm{f}}\right)$ is relatively small because only a small number of droplets are being formed in entropic terms. Hence, the larger interfacial area variable results in an overall $\Delta \mathrm{G}_{\mathrm{f}}$ $\geq 0$. Nonetheless, emulsions are kinetically stable due to the presence of an adsorbed interfacial layer which may include ionic surfactants (electrostatic forces) or non-ionic surfactants (steric forces) (Taylor 1998). 
To reduce the emulsion droplet size, energy is required to overcome its Laplace pressure which is the pressure differential between the convex and concave side of the interface:

$$
\text { Laplace pressure }\left(p_{L}\right)=\gamma\left(\frac{1}{R_{1}}+\frac{1}{R_{2}}\right)
$$

where $\mathrm{p}_{\mathrm{L}}$ is the Laplace pressure, $\mathrm{R}_{1}$ and $\mathrm{R}_{2}$ are the radii. Accordingly, a smaller droplet radius corresponds to a greater pressure needed to be overcome to achieve further particle size reduction; however, by lowering the surface tension, via the addition of surfactants, the Laplace pressure also decreases. The process of emulsification involves: droplet deformation and breakdown, transportation and adsorption of surfactants to the newly formed droplet interface, and droplets possibly colliding and coalescing. As these processes occur simultaneously, a steady-state is not necessarily achieved. Ultimately, the main functions of surfactants are to (1) lower interfacial tension to facilitate a droplet size reduction and (2) prevent recoalescence of droplets (Walstra 1993).

\subsection{Prevention of Emulsion Destabilization}

Without appropriate controls or after a prolonged duration, an emulsion will eventually separate into its two distinct phases. There are four primary mechanisms which destabilize an emulsion: creaming (gravitational separation), aggregation, coalescence, and Ostwald ripening. Typically, these mechanisms occur in combination (Figure 3.1) (Fredrick et al. 2010). 


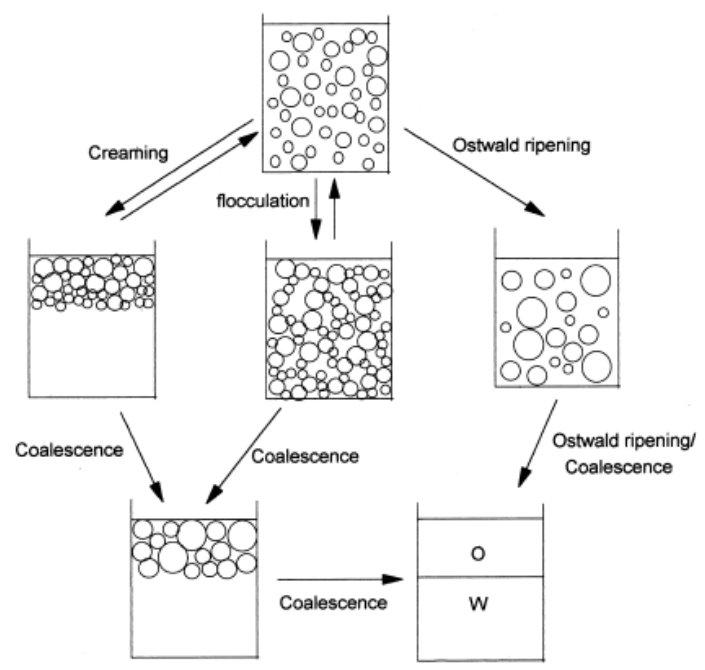

Figure 3.1 The relationships between different o/w emulsion destabilization mechanisms. Reprinted with permission from Meinders, M. B. J.; Kloek, W.; Van Vliet, T. Effect of Surface Elasticity on Ostwald Ripening in Emulsions. Langmuir 2001, 17, 3923-3929. Copyright 2001 American Chemical Society.

Perhaps the most straightforward destabilization mechanism, creaming of an o/w emulsion, involves the gravitational separation of the dispersed oil and continuous aqueous phases due to differences in density (Rousseau 2000). For example, after some time, milk fat droplets in unprocessed raw milk will rise and gather together, forming a cream layer at the top (Fredrick et al. 2010). An effective method of stabilizing against creaming is to simply reduce average droplet size and increase the uniformity of the droplets via homogenization (Rousseau 2000).

Aggregation occurs when droplets come into contact, but whether they remain in contact depends on internal (i.e., attraction/repulsion) and external forces (i.e., stirring/agitation) (Fredrick et al. 2010). Flocculation is the aggregation of particles due to weak attractive forces, in which droplets retain their structural integrity. It is also described by the DLVO (Derjaguin-Landau, Verwey-Overbeek) theory as a function of interparticle distance and the balance between attraction (London-van der Waals forces) and repulsion (surfactants) (Rousseau 2000). Emulsions stabilized by globular proteins are stable against flocculation if no salt is present - due to electrostatic screening, but this is not usually the case in foods (Zhai et al. 2013). As flocculation involves 
weaker associations, it is easier to disrupt the aggregates and may be reversible. However, when stronger forces are required to disrupt aggregates, this form of aggregation is referred to as coagulation (Fredrick et al. 2010).

If two droplets remain in close contact for long enough, the film separating them may rupture (Fredrick et al. 2010). Creaming and flocculation are both precursors to coalescence as they bring droplets close together (Taylor 1998). The likelihood of film rupture increases with larger droplet size, thinner film (greater attraction between droplets), and lower interfacial tension (which depends on the surfactant used). Coalescence results in the formation of one larger droplet from two smaller droplets, which assumes a spherical shape as this has the lowest Laplace pressure (Fredrick et al. 2010). To prevent coalescence, surfactants must form a viscous and rigid film at interface that acts as a physical barrier. While complete coalescence occurs between liquid droplets; the presence of crystalline matter within droplets results in partial coalescence (Rousseau 2000). A partly-crystalline oil phase allows for the formation of a crystal network within oil droplets, with some crystal protrusion into the aqueous phase (Fredrick et al. 2010).

Ostwald ripening and coalescence are both examples of "coarsening phenomena". Coalescence can be prevented by using certain surfactants; Ostwald ripening may occur whenever curved interfaces are present (Meinders et al. 2001). Ostwald ripening does not require droplets to be in close proximity, as it is based on diffusion (Taylor 1998). It involves the preferential diffusion of small droplets into larger droplets, due to their greater Laplace pressure (Bibette et al. 1992). The solubility of dispersed droplets increases with decreasing radius of curvature, smaller droplets tend to dissolve into the bulk phase and preferentially diffuse to larger droplets. This is because by growing larger to minimize interfacial area, emulsions droplets can reduce their free energy 
(Taylor 1998). A critical radius $\left(\mathrm{C}_{\mathrm{r}}\right)$ determines which droplets shrink (radius $<\mathrm{C}_{\mathrm{r}}$ ) and which droplets grow (radius $>\mathrm{C}_{\mathrm{r}}$ ) (Meinders et al. 2001).

\subsection{Hydrophilic-Lipophilic Balance of Surfactants}

The hydrophilic-lipophilic balance (HLB), often used to describe surfactants, is calculated from the weight percentage of the hydrophilic groups to the hydrophobic groups in a molecule, with values ranging from 1-20 (Kralova and Sjöblom 2009). The HLB value of a surfactant should match the HLB value of the oil phase based on the notion of "like dissolves like". It is not a simple linear relationship in which functionality increases with higher hydrophobicity (Nakai 1983).

As mentioned previously, the type of emulsion formed (o/w or w/o) is mainly determined by the surfactant present (Walstra 1993). Surfactants with an HLB value between 3-6 (i.e., glycerol esters, propylene glycol fatty acid esters, polyglycerol esters, and sorbitol fatty acid esters) promote formation of w/o emulsions. Surfactants with HLB values between 8-16 tend to form o/w emulsions. Surfactants between 8-16 include: proteins, phospholipids, potassium and sodium salts, and alginates (Kralova and Sjöblom 2009).

Nevertheless, there are limitations to the HLB concept. Depending on temperature, a single emulsifier can produce either an o/w or w/o emulsion. At high oil concentrations, a single emulsifier can produce an o/w at low emulsifier concentration, or a w/o emulsion at high emulsifier concentration. Furthermore, o/w emulsions can be prepared with emulsifiers across the entire HLB range. The stability of an emulsion prepared with a blend of opposite emulsifiers (i.e., Spans and Tweens) will depend on how the emulsifiers are positioned at the interface. Lastly, the theoretical calculation of HLB values does not apply to blends of emulsifiers that associate at the oil-water interface (Boyd et al. 1972). 


\subsection{Gibbs-Marangoni Effect}

The Gibbs-Marangoni effect describes the mechanism that prevents recoalescence of newly formed emulsion droplets (Figure 3.2). When two droplets that are insufficiently coated with surfactant move towards each other, they will accumulate more surfactants as they approach each other. But, the amount of surfactant available for adsorption will be lowest at the point where the droplets are closest. This creates an interfacial tension $(\gamma)$ gradient, where $\gamma$ is highest at this point. Due to this gradient, surfactants will move in the direction of highest $\gamma$, where there is the least amount surfactant coverage. This streaming of liquid along the surface is known as the Marangoni effect, a self-stabilizing mechanism in which droplets are driven away from each other. However, this only occurs if the surfactant is present in the continuous phase and before the system reaches equilibrium. According to Bancroft's Rule, when forming an emulsion, the phase in which the surfactant is most soluble becomes the continuous phase. The efficacy of the Gibbs-Marangoni mechanism depends on Gibbs elasticity (E) of film, where (A) represents area.

$$
\varepsilon=\frac{2 d \gamma}{d \ln A}
$$

If $\mathrm{E}$ is high, there is a strong interfacial gradient and stabilization occurs (Walstra 1993). Interfacial layers formed by non-protein surfactants are stabilized by the Gibbs-Marangoni effect. This mechanism relies on rapid diffusion of surfactants to the interface to prevent surface concentration gradients (Wilde et al. 2004). 


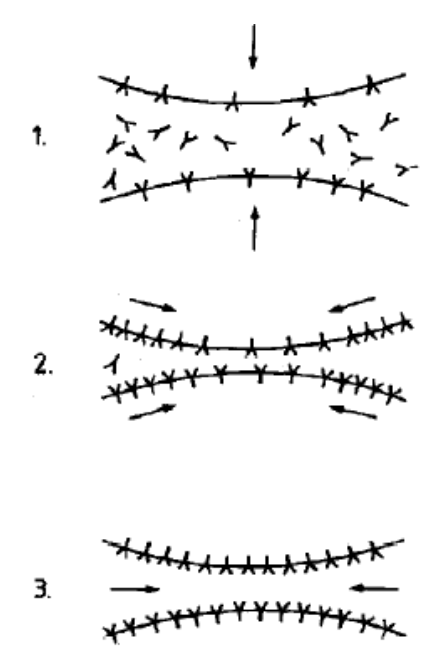

Figure 3.2 Portrayal of the Gibbs-Marangoni effect on two approaching emulsion droplets, with surfactants represented by Y. Reprinted with permission from Walstra, P. Principles of emulsion formation. Chem. Eng. Sci. 1993, 48 (2), 333-349. Copyright 1993 Elsevier.

\subsection{Critical Micelle Concentration (CMC)}

As surfactants are amphiphilic structures with a non-polar hydrocarbon tail and polar (usually ionic) head group, they arrange themselves into micelles in polar solvents, such as water. This is due to the hydrophobic effect, in which surfactants arrange themselves into a micellar structure with a hydrophobic core and hydrophilic head groups at the interface. The critical micelle concentration (CMC) refers to the concentration of surfactant above which micelles will form. There are three common methods of CMC determination: (1) measure the change in UV-vis spectrum of benzoylacetone, (2) measure the fluorescence emission spectrum of pyrene monomers, or (3) measure the electrical conductivity of an ionic surfactant solution as concentration increases. To determine CMC, simply plot the physical property as a function of surfactant concentration and the $\mathrm{CMC}$ will be the point in which the slope changes. $\mathrm{CMC}$ decreases with increasing hydrocarbon chain length of non-polar groups, or addition of electrolytes for ionic surfactants (Domínguez et al. 1997). 


\subsection{Major Food Surfactants}

\subsubsection{Low Molecular Weight Surfactants}

Low molecular weight (LMW) surfactants are very mobile at the interface making them efficient at lowering interfacial tension (Kralova and Sjöblom 2009). LMW emulsifiers do not form a viscoelastic interfacial layer, but rather form a compact interfacial layer. Competitive destabilization may occur when both proteins and surfactants are present at the interface. Surfactants weaken protein interactions, while proteins hinder surfactant mobility (GibbsMarangoni effect) (Wilde et al. 2004). As LMW surfactants are smaller than proteins, they tend to displace proteins from the interface (Rousseau 2000). At low surfactant concentration, proteinsurfactant complexes form. While, high surfactant concentration results in protein displacement from the interface. Therefore, LMW surfactants are commonly used to intentionally destabilize food emulsions, such as ice cream, allowing for partial coalescence (Dickinson et al. 1989).

Lecithin used in foods are a mixture of phospholipids and can be modified to obtain various HLB values (van Nieuwenhuyzen and Szuhaj 1998). Methods of modification include fractionation in alcohol, hydrolysis (enzymatic, acid or alkali), acetylation or hydroxylation. Lecithin can be derived from egg yolk or various oilseeds (soybean, flax, cottonseed, corn, rapeseed, sunflower). However, soybean is the most common source due to its availability, emulsifying properties, colour, and taste. There are many food applications of soy lecithin, such as: viscosity lowering in chocolate, protein interaction in baked products, retardation of fat crystallization in chocolate, and dispersing effect in instant drinks. Furthermore, its stability increases when used together with other surfactants, such as monoglycerides or proteins (Van Nieuwenhuyzen 1976). 
Mono- and di-glycerides are glycerol esters of fatty acids. They are most commonly used in the food industry for bakery products (i.e., bread, cake, cake mixes) and frozen desserts (Weiss 1971). Monoglycerides have two isomeric forms (1-form and 2-form) based on a temperaturedependent equilibrium (Lauridsen 1976). Sorbitan esters, also known as "Spans", are products of a reaction between sorbitol and fatty acids. Polysorbates, also known as "Tweens" are polyoxyethylene derivatives of sorbitan esters. Spans and Tweens are often used together in cakes, cake mixes, whipped toppings, icings, and coffee whiteners (Weiss 1971). According to Boyd et al. (1972), optimum stability in o/w emulsions is achieved when using a 1:1 ratio of Spans and Tweens (Boyd et al. 1972).

\subsubsection{Proteins}

The main emulsion stabilization mechanism of proteins is through the formation of a viscoelastic adsorbed layer. However, the extent of adsorption depends on protein surface hydrophobicity and charge (Wilde et al. 2004). As non-polar amino acids reside within protein molecules to avoid contact with aqueous surroundings, only some of them partake in the emulsification process. Thus, the term protein hydrophobicity typically refers to surface hydrophobicity. As emulsifiers, proteins adsorb to the interface due to their amphiphilic nature and form a thick coating around oil droplets. The emulsifying ability of proteins depends on a balance between hydrophilic and lipophilic portions (Nakai 1983). As shown in Figure 3.3, when protein hydrophobicity increases, interfacial tension decreases and emulsifying activity increases. Thus, greater exposure of hydrophobic residues on proteins improves emulsifying and foaming ability. Additionally, the effectiveness of a protein as an emulsifier is also attributed to its molecular flexibility. For example, casein molecules are better emulsifiers than whey proteins as they have a looser conformation (Nakai 1983). 


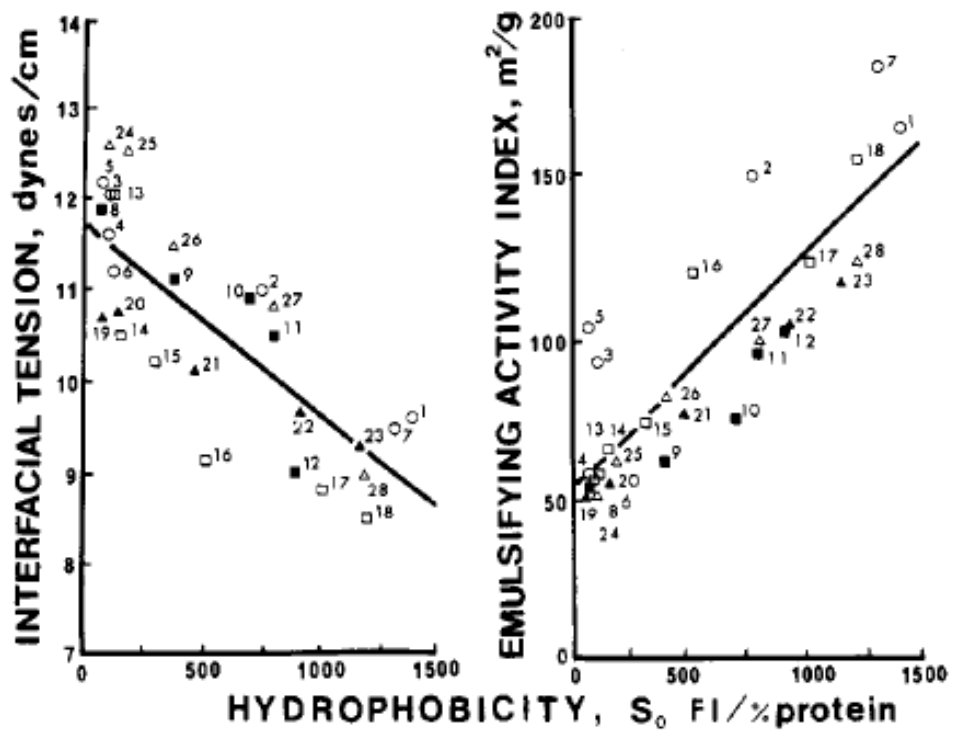

Figure 3.3 Relationship between interfacial tension, surface hydrophobicity and emulsifying activity of various proteins. Reprinted with permission from Nakai, S. Structure-Function Relationships of Food Proteins with an Emphasis on the Importance of Protein Hydrophobicity. $J$. Agric. Food Chem. 1983, 31 (4), 676-683. Copyright 1983 American Chemical Society.

\subsubsection{Applications in Food Systems}

Cream liqueurs are o/w emulsions that contain ethanol in its aqueous continuous phase. The presence of ethanol (up to $20 \%$ wt.) improves emulsification and produces finer droplets, as it lowers interfacial tension. However, increasing alcohol content may eventually lead to protein aggregation, with precipitation observed at $>30 \%$ wt. ethanol (Dickinson et al. 1989). Sodium caseinate is the primary stabilizer in cream liqueurs (Kralova and Sjöblom 2009). Sodium caseinate forms an interfacial layer of caseinate submicelles around fat droplets to stabilize emulsions (Rousseau 2000). If LMW surfactants are added to cream liqueurs, they reside in the primary interfacial layer with ethanol; while, the secondary interfacial layers contain adsorbed caseinates. But due to the intrinsic emulsifying ability of cream liqueurs, the addition of low MW surfactants does not significantly influence droplet size distribution. Ethanol content also lowers the surface viscosity of adsorbed caseinate film at the interface. This is beneficial for the consumer 
acceptance of cream liqueurs which should not be too viscous or viscoelastic (Dickinson et al. 1989).

Ice cream and whipped toppings are both examples of partially-coalesced emulsions that undergo shear and incorporation of small air bubbles. Whipped products use surfactants to displace proteins and weaken the interfacial layer, allowing oil from emulsion droplets to spread into the air-water interface (Dalgleish 2006). Compared to whipped products, ice cream has a lower fat volume, presence of ice crystals, and also utilizes a different whipping process (Petrut et al. 2016). For these products, it is important that the fat phase is semi-liquid during whipping, but crystallizes afterwards (Dalgleish 2006), The purpose of fat network formation is to entrap air bubbles and stabilize the foam structure (Petrut et al. 2016).

In ice cream, the destabilization of fat emulsions during whipping and subsequent freezing is necessary to impart dryness during extrusion, smooth mouthfeel/texture, and resistance to meltdown. Ice cream has an aqueous phase composed of sugar, salt, and a colloidal casein suspension. Its discrete globular phase is partially-crystalline and contains triglycerides, diglycerides and monoglycerides. The interface contains milk proteins, phospholipids, lipoprotein particles and any added surfactants (Goff and Jordan 1989).

Low HLB surfactants are not effective destabilizers as they adsorb to the fat globules, which helps retain their structural integrity. High HLB emulsifiers do not associate with fat globules as strongly and are easily displaced during agitation. This exposes the fat globules and promotes coalescence. For example, Tweens $(\mathrm{HLB}=15)$ are very effective destabilizers, while Spans (HLB = 4.3-8.6) are not (Goff and Jordan 1989). Casein stabilized emulsions are more stable against partial coalescence than whey proteins; however, caseins are more easily displaced by LMW surfactants. This protein displacement mechanism is important in lowering emulsion 
stability to reduce viscoelasticity and thickness of interface (Fredrick et al. 2010). Typically, monoglycerides and diglycerides are used to induce partial coalescence, while polysaccharides (such as carrageenan and gums) are used as stabilizers in ice cream (Kralova and Sjöblom 2009).

Mayonnaise contains up to $70-80 \%$ fat, and is considered an acidic o/w emulsion. The low $\mathrm{pH}$ and high fat content of mayonnaise makes it highly resistant to microbial spoilage (Depree and Savage 2001). To maintain emulsion stability, there must not be fat crystals within the dispersed oil phase. The aqueous continuous phase typically consists of eggs, sugar, vinegar, salt and spices. Egg yolk contains lecithin that function as the emulsifier and stabilizer (van Nieuwenhuyzen and Szuhaj 1998). Salt is also an important ingredient as it helps disperse egg yolk granules and neutralizes the charged proteins. This allows proteins to strongly adsorb to the interface and better interact with adjacent droplets, as shown below in Figure 3.4 (Depree and Savage 2001).

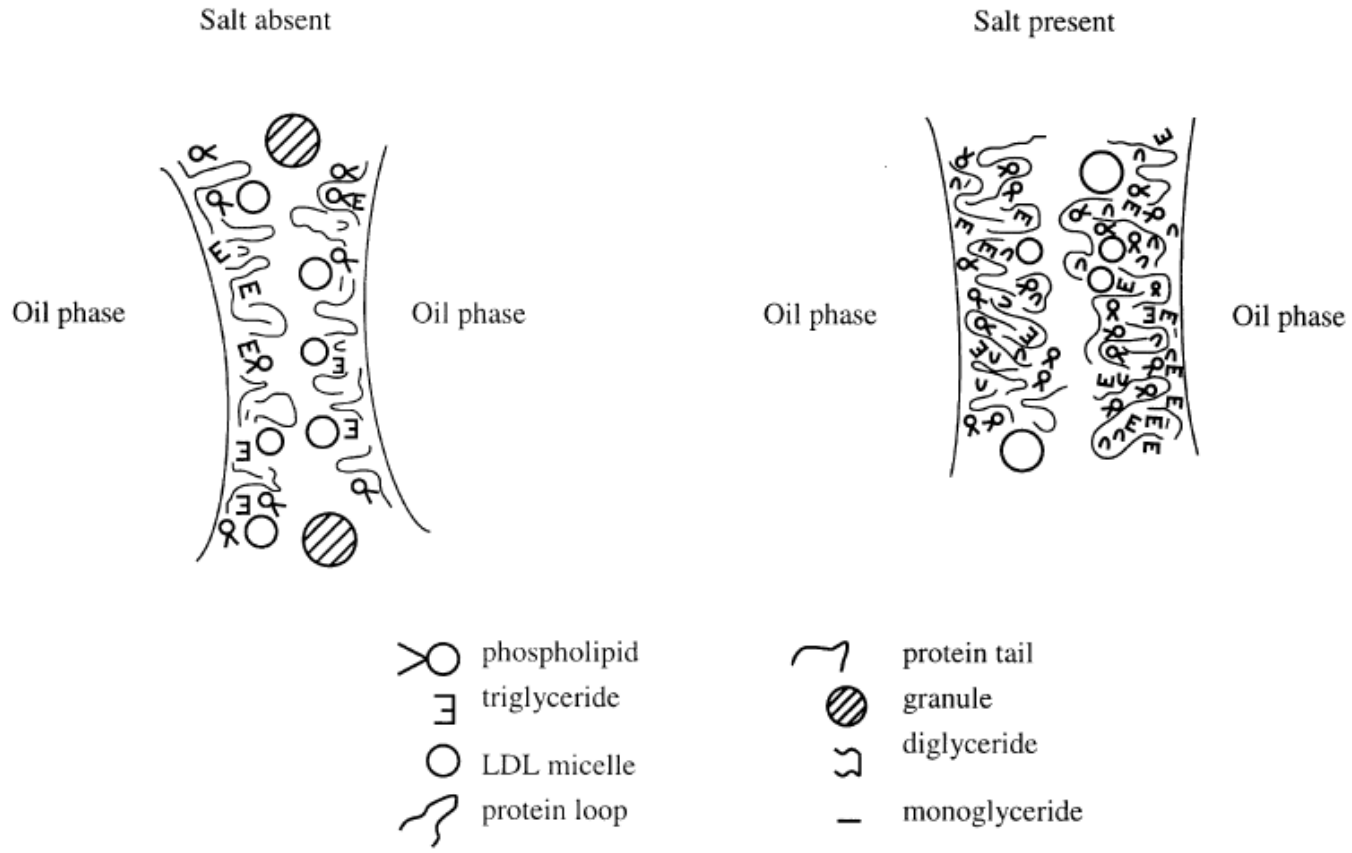

Figure 3.4 The interfacial components and effect of salts on mayonnaise o/w emulsion. Reprinted with permission from Depree, J. A.; Savage, G. P. Physical and flavour stability of mayonnaise. Trends Food Sci. Technol. 2001, 12 (5-6), 157-163. Copyright 2002 Elsevier Science Ltd. 
To make mayonnaise, the first step is to mix together the components of the aqueous phase. Next, oil must be gradually added while continuously blending. This is an important step as the mixing of both phases together all at once will result in the formation of a w/o emulsion. Oil droplets within mayonnaise are distorted from their normal spherical shape as they are tightly associated with each other. This flocculation is important for mayonnaise viscosity and texture. Consequently, the $\mathrm{pH}$ of mayonnaise should be near the isoelectric point of the egg yolk proteins ( 3.9) to allow for maximum viscoelasticity and stability (Depree and Savage 2001). This partialphase inversion results in a heat stable emulsion that can be pasteurized. Heating up mayonnaise to $60^{\circ} \mathrm{C}$ denatures the egg proteins, followed by hydrolysis to produce phosphatidylcholine which is an effective o/w surfactant. Alternatively, hydrolyzed soy lecithin is also commonly used in mayonnaise (van Nieuwenhuyzen and Szuhaj 1998).

\subsection{Conclusion}

Overall, surfactants have a multitude of applications within the food processing industry. As emulsions are thermodynamically unstable, these surface-active agents are employed to improve kinetic stability and increase product shelf-life. Emulsions may be destabilized via creaming (gravitational separation), aggregation, coalescence, and/or Ostwald ripening. However, surfactants may combat these processes through several means. Electrostatic interactions involve the surface charge of emulsions droplets which is influenced by the presence of ionic surfactants, proteins and other charged compounds. Low molecular weight surfactants have the ability to displace proteins to induce partial coalescence which is desired in promoting foam stability of ice cream and whipped toppings. Lastly, dispersed emulsion droplets may also be stabilized by the steric repulsive forces of mono- and di-glycerides or proteins. 


\subsection{References}

Bibette J, Morse DC, Witten TA, Weitz DA (1992) Stability criteria for emulsions. Phys Rev Lett 69:2439-2442 . doi: 10.1103/PhysRevLett.69.2439

Boyd J, Parkinson C, Sherman P (1972) Factors affecting emulsion stability, and the HLB concept. J Colloid Interface Sci 41:359-370 . doi: 10.1016/0021-9797(72)90122-1

Dalgleish DG (2006) Food emulsions - Their structures and structure-forming properties. Food Hydrocoll 20:415-422 . doi: 10.1016/j.foodhyd.2005.10.009

Depree JA, Savage GP (2001) Physical and flavour stability of mayonnaise. Trends Food Sci Technol 12:157-163 . doi: 10.1016/S0924-2244(01)00079-6

Dickinson E, Narhan SK, Stainsby G (1989) Stability of Cream Liqueurs Containing LowMolecular-Weight Surfactants. J Food Sci 54:77-81 . doi: 10.1111/j.13652621.1989.tb08572.x

Domínguez A, Fernández A, González N, et al (1997) Determination of Critical Micelle Concentration of Some Surfactants by Three Techniques. J Chem Educ 74:1227-1231 . doi: 10.1021/ed074p1227

Fredrick E, Walstra P, Dewettinck K (2010) Factors governing partial coalescence in oil-in-water emulsions. Adv Colloid Interface Sci 153:30-42 . doi: 10.1016/j.cis.2009.10.003

Goff HD, Jordan WK (1989) Action of Emulsifers in Promoting Fat Destabilization During the Manufacture of Ice Cream. J Dairy Sci 72:18-29 . doi: 10.3168/jds.S0022-0302(89)79075-5

Kralova I, Sjöblom J (2009) Surfactants used in food industry: A review. J Dispers Sci Technol 30:1363-1383 . doi: 10.1080/01932690902735561

Lauridsen JB (1976) Food emulsifiers: Surface activity, edibility, manufacture, composition, and application. J Am Oil Chem Soc 53:400-407 . doi: 10.1007/BF02605731

Meinders MBJ, Kloek W, Van Vliet T (2001) Effect of Surface Elasticity on Ostwald Ripening in Emulsions. Langmuir 17:3923-3929 . doi: 10.1021/la000304z

Nakai S (1983) Structure-Function Relationships of Food Proteins with an Emphasis on the Importance of Protein Hydrophobicity. J Agric Food Chem 31:676-683 . doi: 10.1021/jf00118a001

Petrut RF, Danthine S, Blecker C (2016) Assessment of partial coalescence in whippable oil-inwater food emulsions. Adv Colloid Interface Sci 229:25-33 . doi: 10.1016/j.cis.2015.12.004

Rousseau D (2000) Fat crystals and emulsion stability - A review. Food Res Int 33:3-14 . doi: 10.1016/S0963-9969(00)00017-X

Taylor P (1998) Ostwald ripening in emulsions. Adv Colloid Interface Sci 75:107-163 . doi: 10.1016/S0001-8686(98)00035-9

Van Nieuwenhuyzen W (1976) Lecithin production and properties. J Am Oil Chem Soc 53:425427 . doi: 10.1007/BF02605737

van Nieuwenhuyzen W, Szuhaj BF (1998) Effects of lecithins and proteins on the stability of emulsions. Lipid - Fett 100:282-291 . doi: 10.1002/(SICI)1521-4133(199807)

Walstra P (1993) Principles of emulsion formation. Chem Eng Sci 48:333-349 . doi: 10.1016/0009-2509(93)80021-H

Weiss TJ (1971) Food emulsifiers-Science and art. J Am Oil Chem Soc 49:457-461

Wilde P, Mackie A, Husband F, et al (2004) Proteins and emulsifiers at liquid interfaces. Adv Colloid Interface Sci 108-109:63-71 . doi: 10.1016/j.cis.2003.10.011

Zhai J li, Day L, Aguilar MI, Wooster TJ (2013) Protein folding at emulsion oil/water interfaces.

Curr Opin Colloid Interface Sci 18:257-271 . doi: 10.1016/j.cocis.2013.03.002 


\section{Chapter 4 Lipid Digestion of Oil-in-Water Emulsions Stabilized with Low Molecular Weight Surfactants ${ }^{2}$}

\subsection{Abstract}

Surfactant chemistry and concentration influences lipid digestion and thus total fatty acid bioaccessibility, as determined in the state-of-the-art simulated robotic TIM-1 gastrointestinal (GI) tract. Comparisons between surfactants (monoglycerides, (i.e., glycerol monostearate (1-GMS), glycerol monooleate (1-GMO), and $s n-2$ glycerol monooleate (2-GMO)), or non-ionic synthetic surfactants (sorbitan monostearate (Span® 60), sorbitan monooleate (Span® 80), polyoxyethylene (20) sorbitan monostearate (Tween® 60), or polyoxyethylene (20) sorbitan monooleate (Tween ${ }^{\circledR}$ 80)) were evaluated in oil-in-water emulsions. The minimum surfactant concentration to achieve stable (i.e. over a 7-day period) monomodal oil-in-water emulsions with monolayer coverage formed emulsions with an average particle size of $230 \pm 30 \mathrm{~nm}$. Although statistically significant differences in induction time and rate of lipid digestion are not observed, total bioaccessibility was significantly lower for emulsions stabilized with Span 80 and 2-GMO. Comparable trends were observed between area under the curve (AUC) of the absolute bioaccessibility and total overall bioaccessibility obtained by fitting a three-parameter shifted logistic model to the cumulative bioaccessibility. Significantly $(p<0.05)$ lower overall lipid bioaccessibility was observed for Span 80- and 2-GMO-stabilized oil-in-water emulsions compared to all other surfactants studied. No significant differences for overall bioaccessibility were observed between oil-in-water emulsions stabilized by commercial 1-GMO and 1-GMS, Span 60, Tween 60 and Tween 80 . The results confirm that sn-position and surfactant choice can influence the lipid digestion (total

\footnotetext{
${ }^{2}$ Manuscript submitted to Food \& Function, Royal Society of Chemistry Journals (July $1^{\text {st }}, 2019$ )
} 
bioaccessibility) in vitro. This provides an exciting strategy to tailor lipemic responses of food emulsions.

\subsection{Introduction}

Ultra-processed foods (UPFs) account for $>50 \%$ of the total dietary energy intake in developed countries (i.e., USA, Canada, and the UK) and 20-30\% in middle-income countries (i.e., Mexico, Chile, and Brazil) (Moubarac et al. 2017; Baraldi et al. 2018; Cediel et al. 2018; Louzada et al. 2018; Marrón-Ponce et al. 2018; Rauber et al. 2018; Monteiro et al. 2019). Common benefits of UPFs include: extended shelf-stability, hyper-palatability, convenience and low-cost, however for the most part they are obesogenic (Moubarac et al. 2013, 2014; Monteiro et al. 2013, 2019). UPFs are typically high in saturated or trans fats, refined sugars, and/or salt with high energy density of UPFs ranges from 225-275 kcal per $100 \mathrm{~g}$ of most baked products to 400-500 kcal per $100 \mathrm{~g}$ of most packaged snack products and chips (Monteiro et al. 2018a). Numerous studies correlate frequency of UPF consumption with increased obesity prevalence (Louzada et al. 2015; Juul and Hemmingsson 2015; Poti et al. 2017; Monteiro et al. 2018b; Nardocci et al. 2019). For example, Canadian adults in the highest quintile of UPF consumption ( $76 \%$ of total energy intake) were reported to have $32 \%$ greater occurrence of obesity compared to individuals in the lowest quintile (20\% of total energy intake) (Nardocci et al. 2019). Compounding the issue, being overweight (BMI $\geq 25$ to $<30 \mathrm{~kg} / \mathrm{m}^{2}$ ) or obese $\left(\mathrm{BMI}>30 \mathrm{~kg} / \mathrm{m}^{2}\right.$ ) is strongly associated with increased risk of diet-related non-communicable diseases (i.e., coronary heart disease, stroke, etc.); obese individuals exhibited the highest risk, having reported hazard ratios of 1.69 for coronary heart disease and 1.47 for stroke compared to normal weight individuals (BMI $\geq 20$ to $<25 \mathrm{~kg} / \mathrm{m}^{2}$ ) (Lu et al. 2014). Consequently, dietary risk factors have become the leading cause of all-cause 
mortality, with $~ 11$ million deaths across 195 countries attributable to dietary risk factors in 2017; this represents a new and complex form of malnutrition unseen 100 years ago (Afshin et al. 2019).

Careful attention to food processing and formulation is a potential avenue to attenuate negative health outcomes associated with diets largely dominated by UPFs (Monteiro et al. 2018a). In addition to composition, the physical structure of foods also impacts digestion and consequently their metabolic response (Golding et al. 2011). Food matrices are complex, heterogeneous combinations of solids, liquids and/or gases that may either occur naturally or form as a result of processing and formulation (Guo et al. 2017). Since food matrices influence bioavailability, understanding the relationship between food structure and digestion facilitates design of healthier processed foods. For example, healthier processed foods may be engineered to control lipid digestion kinetics, modifying their bioavailability and potentially reducing their lipemic index (McClements et al. 2008, 2009; Singh et al. 2009; Singh and Sarkar 2011; Guo et al. 2017; Dias et al. 2019). The lipemic index is similar to the well-established glycemic index and represents post-prandial serum triacylglyceride (TAG) levels following ingestion of a meal relative to a reference meal, which contains a fixed load (i.e., 50-70 g) of saturated, polyunsaturated and monounsaturated fats in known quantities (Ooi et al. 2011). Elevated post-prandial lipemia is a criterion for metabolic syndrome and is recognized as a risk factor for obesity-related chronic diseases, such as type-2 diabetes and cardiovascular diseases (Talmud et al. 2002; Patel 2004; Bansal et al. 2007; Nordestgaard et al. 2007; Sarwar et al. 2007; Dekker et al. 2009). Individuals with higher concentrations of non-fasting TAGs ( $\geq 5 \mathrm{mmol} / \mathrm{L}$ ) are associated with higher risk of heart failure (hazard ratio of 2.59) compared to individuals with concentrations $<1 \mathrm{mmol} / \mathrm{L}$, as shown in a large prospective cohort study of the general Copenhagen population (Mortensen et al. 2015; Varbo and Nordestgaard 2018). 
During digestion, lipolysis cleaves water-insoluble TAGs into amphiphilic lipolytic products (i.e., 1,2- and 2,3-diacylglycerides (DAGs), 2-monoacylglycerides (2-MAGs), and free fatty acids (FFAs)), in the oral, gastric and intestinal segments of the GI tract (Stipanuk 2006; Hur et al. 2009; Guo et al. 2017). Lipolysis is initiated in the stomach by acid-stable lingual and gastric lipases (Moreau et al. 1988; Wahbeh and Christie 2011). In adults, lingual lipase secreted by the serous Von Ebner glands of the tongue does not significantly contribute to overall lipolysis; lingual lipase is more significant in infants or patients with severe pancreatic insufficiency (Abrams et al. 1987; Hamosh 1990; Mu and Høy 2004). Gastric lipase is largely responsible for pre-duodenal lipolytic activity $(\sim 118 \mathrm{U} / \mathrm{g})$, while lingual lipase is only present in trace amounts $(\sim 0.3 \mathrm{U} / \mathrm{g})$ (DeNigris et al. 1988). Gastric lipase cleaves sn-3 ester bonds of TAGs, resulting in the formation of 1,2-DAGs and FFAs (Singh and Ye 2013). Overall, gastric lipolytic activity hydrolyzes $~ 10-$ $30 \%$ of ingested TAGs in human adults (Wahbeh and Christie 2011; Singh and Ye 2013). Gastric lipolytic products act as emulsifying agents facilitating digestion in the small intestine where lipid absorption primarily occurs (Carey et al. 1983; Lien 1994). Pancreatic lipase is responsible for the majority of lipolysis ( 40-70\% of TAG lipolysis) and cleaves both $s n-1$ and $s n-3$ ester bonds, producing 2-MAGs and FFAs as lipolytic metabolites (Reis et al. 2010; Wilde and Chu 2011; Meynier and Genot 2017). Pancreatic lipase requires co-lipase to reduce interfacial tension facilitating adsorption of lipase at oil-water interface in the presence of bile salts (Wilde and Chu 2011). Bile then displaces surface-active metabolites from the interface (Reis et al. 2008b). Lipolytic products must be incorporated into mixed micelles $(8-20 \mathrm{~nm})$ containing bile salts or larger vesicles $(40-200 \mathrm{~nm})$ containing phospholipids that then absorb via junctions between enterocytes (Meynier and Genot 2017). Therefore, the rate of lipid digestion depends on: 1) the accessibility of lipase/co-lipase at the oil-water interface, 2) competitive adsorption between bile 
salts; and, 3) surface-active materials and displacement of surface-active compounds with the aid of bile salts (Reis et al. 2009; Wilde and Chu 2011; Singh and Ye 2013; Speranza et al. 2013).

Low molecular weight surfactants are capable of displacing proteins, including lipolytic enzymes, from the oil-water interface by competing for adsorption at the oil-water interface, which potentially modifies lipolysis kinetics (Vinarov et al. 2012). Surfactant monolayers surrounding oil droplets must first be displaced by bile to facilitate lipase absorption onto the interface and lipolytic activity to occur; thus, surfactants have the potential to tailor lipid bioaccessibility (Speranza et al. 2013). Enzyme-surfactant interactions are dependent on concentration and surfactant selection (Reis et al. 2008a, 2010). Accumulation of surface-active lipolytic products at the oil-water interface occurs when the rate of hydrolysis exceeds the micellization rate (Carey et al. 1983; Reis et al. 2010). The complexity and dynamic nature of the interfacial environment makes it difficult to understand and quantify lipolysis dynamics (Reis et al. 2010). As such, a better understanding regarding the effect of surfactants on lipase activity is needed, including enzyme interactions with endogenous surface-active compounds generated in situ from lipolysis, such as 2-MAGs (Reis et al. 2010; Vinarov et al. 2012). As surfactants are widely used in the food industry to increase emulsion stability, it is imperative to consider their physiological impact on digestion (Speranza et al. 2013; McClements 2018). The purpose of this study was to investigate the effects of $s n$ - fatty acid position of monoglycerides and structure of non-ionic, synthetic surfactants on the rate and extent of lipid digestion using the TIM-1 advanced, robotic GI model.

\subsection{Materials \& Methods}

\section{Materials}

Lipase from porcine pancreas (type II, 100-500 U/mg protein), pancreatin from porcine pancreas ( >100 U/mg protein), porcine bile extract, bovine pancreas trypsin powder $(\geq 7,500 \mathrm{~N}$ - 
$\alpha$-benzoyl-L-arginine ethyl ester (BAEE) $\mathrm{U} / \mathrm{mg}$ ), pepsin from porcine gastric mucosa $(\geq 2,500$ $\mathrm{U} / \mathrm{mg}$ protein) and $\alpha$-amylase from Bacillus $s p$. (type II-A, $\geq 1,500 \mathrm{U} / \mathrm{mg}$ protein) were obtained from Sigma Aldrich (St. Louis, MO, USA). Fresh porcine bile was collected at a local slaughterhouse (Conestoga Meat Packers, Breslau, ON, Canada), pooled, aliquoted for individual TIM-1 experiments and stored at $-30^{\circ} \mathrm{C}$ until use. Tricaprylin (>99\%), triolein (>99\%), caprylic acid (>99\%), nonanoic acid ( >97\%), Span ${ }^{\circledR} 80$ (sorbitan monooleate, $\geq 60 \%$ C18:1), Span ${ }^{\circledR} 60$ (sorbitan monostearate, $\sim 50 \% \mathrm{C} 18: 0$ ), Tween ${ }^{\circledR} 80$ (polyoxyethylene sorbitan monooleate, $\geq 58 \%$ C18:1) and Tween ${ }^{\circledR} 60$ (polyoxyethylene sorbitan monostearate, 40-60\% C18:0) were obtained from Sigma Aldrich (St. Louis, MO, USA). Glycerol monostearate (1-GMS, Alphadim® 90 SBK, $>90 \%$ ) was obtained from Corbion N.V. (Amsterdam, The Netherlands). Glycerol monooleate (1GMO, 99\%) was obtained from Alfa Chemistry (Ronkonkoma, NY, USA). Anhydrous ethanol, $\mathrm{HCl}(37 \%)$, anhydrous $\mathrm{NaOH}$, hydroxypropylmethyl cellulose (HPMC), $\mathrm{NaHCO}_{3}(\geq 99 \%), \mathrm{NaCl}$ ( $\geq 99 \%), \mathrm{KCl}(\geq 99 \%)$ and $\mathrm{CaCl}_{2}(\geq 96 \%)$ were obtained from Sigma Aldrich (St. Louis, MO, USA).

Dichloromethane (DCM, >99\%, $50 \mathrm{ppm}$ amylene) was purchased from Alfa Aesar (Haverhill, MA, USA). Acetonitrile (Optima ${ }^{\mathrm{TM}}$, HPLC grade) and n-heptane (HPLC grade) were purchased from Thermo Fisher Scientific (Waltham, MA, USA).

\section{Synthesis of sn-2 MAGs}

Sn-2 glycerol monooleate (2-GMO) was obtained via enzymatic esterification of triolein using 1,3-regiospecific lipase obtained from porcine pancreas as previously described by Zaks et al. (1992) ${ }^{55} .45 \mathrm{~mL}$ ethanol solution (97\% v/v), $4.5 \mathrm{~g}$ triolein and $1.0 \mathrm{~g}$ of lipase were combined in a flask and placed in a shaking water bath at $20^{\circ} \mathrm{C}$ and $150 \mathrm{rpm}$ for $96 \mathrm{~h}$ and then filtered (Whatman Qualitative Filter Paper Grade 1, >11 $\mu$ m particle retention, Maidstone, UK). An oily residue was obtained by removing ethanol via rotary evaporation at $30^{\circ} \mathrm{C}$ under vacuum $(\sim 400$ 
$\mathrm{mm} \mathrm{Hg}$ ) (Büchi, Essen, Germany) and it was then dissolved in $30 \mathrm{~mL}$-heptane at ambient temperature $\left(22-25^{\circ} \mathrm{C}\right)$ and cooled to $-18^{\circ} \mathrm{C}$ for $>5 \mathrm{~h}$ to initiate crystallization. The crystals were separated via centrifugation at $10500 \times g$ for $3 \mathrm{~min}$ at $-10^{\circ} \mathrm{C}$ (Sorvall LYNX 6000 , Thermo Fisher Scientific, Waltham, MA, USA). The discarded supernatant contained ethyl oleate and diolein; the remaining material containing the crystallized fraction was re-dissolved in $30 \mathrm{~mL}$-heptane and re-cooled to $-18^{\circ} \mathrm{C}$ for $>5 \mathrm{~h}$, crystals were separated again by centrifugation for $3 \mathrm{~min}$ at $-10^{\circ} \mathrm{C}$. DCM was added to facilitate evaporation of residual n-heptane from the crystal fraction using rotary evaporation at $30^{\circ} \mathrm{C}$ under vacuum $(\sim 400 \mathrm{~mm} \mathrm{Hg})$ and was repeated twice. The purified 2GMO ( 97 mol \% of glycerol-containing compounds by ${ }^{1} \mathrm{H}$ NMR, Figure S1 and S2) was stored at $-30^{\circ} \mathrm{C}$ until use.

\section{Emulsion Preparation}

Test meals consisted of an oil-in-water (o/w) emulsion stabilized with either Span® 60, Span ${ }^{\circledR} 80$, Tween ${ }^{\circledR} 60$, Tween ${ }^{\circledR} 80,1-G M S, 1-G M O$ or 2-GMO (Figure 4.1). According to Reis et al., a real food matrix containing additional compounds (i.e., proteins, salts) potentially leads to complexation and interactions that significantly complicate the results often leading to inconclusive data; thus, a system with a single variable was chosen to understand solely the influence of surfactant on lipid digestion profile ${ }^{49}$. Tricaprylin was chosen as the oil fraction based on previous work by Speranza et al. (2013) because its chain length fatty acid is not present in any surfactant or simulated intestinal fluids (as described below); thus, following caprylic acid specifically allows for the measurement of FFA bioaccessibility obtained from their TAG counterpart $^{50}$.

Pre-emulsions were prepared by mixing tricaprylin $(5.0 \% \mathrm{w} / \mathrm{w})$, sodium bicarbonate buffer $(1.5 \mathrm{~g} / \mathrm{L})$ and $0.5 \%(\mathrm{w} / \mathrm{w})$ surfactant (or $0.7 \% \mathrm{w} / \mathrm{w} 2-\mathrm{GMO})$ for 2 min with a high-speed immersion 
blender (KitchenAid, Benton Harbor, MI, USA). Pre-emulsions were then cycled through a highpressure homogenizer (Emulsiflex C-5, Avestin, Ottawa, ON, Canada) 10 times at 50,000 kPa. Immediately after emulsion preparation, the particle size distribution of the emulsion was measured using dynamic light scattering (Nanotrac Flex, Microtrac, Montgomeryville, PA, USA). An aliquot of the emulsion was diluted using distilled water (Milli-Q® Direct Water Purification System, Millipore, Bedford, MA, USA) into a sample vessel to a concentration between the upper and lower limits of the loading index (20-30\%), a measure of the total alternating current (AC) signal obtained from the light scattered from particles moving via Brownian motion. An external probe was inserted and particle size distributions were computed from the measured frequency spectrum recovered from Doppler-shifted scattered light using a refractive index of 1.45 for the dispersed phase (i.e., tricaprylin) (Microtrac $®$ FLEX Windows software, Montgomeryville, PA, USA). Particle size measurements were performed in triplicate over $120 \mathrm{~s}$ at ambient temperature $\left(22-25^{\circ} \mathrm{C}\right)$.

\section{TIM-1 Gastrointestinal Model}

Simulated digestion of emulsions was performed using a state-of-the-art in vitro gastrointestinal model (TIM-1) developed by TNO (Zeist, The Netherlands). The TIM-1 is an advanced, multi-compartmental system that has been validated with in vivo human and animal studies (Souliman et al. 2006; Gervais et al. 2009; Brouwers et al. 2011; Barker et al. 2014; Thilakarathna et al. 2016; Dupont et al. 2018). The TIM-1 models the human digestive system using four serial compartments to simulate dynamic luminal conditions of the stomach, duodenum, jejunum and ileum (Figure 4.2). The system controls numerous aspects of digestion dynamically

(i.e., gastric acidification and emptying, peristaltic movements, nutrient and water absorption, $\mathrm{pH}$ and temperature control $\left(37 \pm 1^{\circ} \mathrm{C}\right)$ and continual secretion of digestive solutions) (Figure 4.2). As 
the TIM-1 digestion model has no hormonal feedback mechanisms (i.e., ileal brake) to regulate gastric emptying, digestion follows a pre-programmed emptying curve (half-time $=80 \mathrm{~min}$; TNO, Zeist, The Netherlands).

Formulated gastric and intestinal solutions were added to each compartment using the fed state protocol, which included: $\mathrm{HCl}(1.00 \mathrm{M})$, sodium bicarbonate $(1.00 \mathrm{M})$, gastric enzyme solution $\left(\mathrm{NaCl} 4.80 \mathrm{~g} / \mathrm{L}, \mathrm{KCl} 2.20 \mathrm{~g} / \mathrm{L}, \mathrm{CaCl}_{2} 0.22 \mathrm{~g} / \mathrm{L}\right.$, lipase $20 \mathrm{U} / \mathrm{mL}$, pepsin $4800 \mathrm{U} / \mathrm{mL}$ and amylase $47 \mathrm{U} / \mathrm{mL})$, gastric solution ( $0.40 \%$ HPMC and $0.04 \%$ bile powder), small intestinal electrolyte solution (SIES: $\mathrm{NaCl} 5.00 \mathrm{~g} / \mathrm{L}, \mathrm{KCl} 0.60 \mathrm{~g} / \mathrm{L}$, and $\mathrm{CaCl}_{2} 0.25 \mathrm{~g} / \mathrm{L}$ ), $7.0 \%$ (w/v) pancreatin solution and fresh porcine bile. Gastric enzyme solution and 7.0\% (w/v) pancreatin solution were stored in cooling units (between $0-5^{\circ} \mathrm{C}$ ) throughout the $5 \mathrm{~h}$ simulated digestion experiments.

Before 'feeding' emulsions to the TIM-1, the system was pre-heated and maintained at physiological temperature $\left(37 \pm 1^{\circ} \mathrm{C}\right)$ and compartments were conditioned with starting residues. Gastric starting residue consisted of $5.0 \mathrm{~g}$ gastric enzyme solution and $5.0 \mathrm{~g}$ gastric solution $(0.40 \%$ HPMC and $0.04 \%$ bile powder). Duodenal starting residue consisted of $15 \mathrm{~g}$ small intestinal electrolyte solution (SIES), $15 \mathrm{~g} 7.0 \%$ (w/v) pancreatin solution, $30 \mathrm{~g}$ fresh porcine bile and $2 \mathrm{mg}$ trypsin solution. Jejunal starting residue contained $35 \mathrm{~g}$ SIES, $35 \mathrm{~g} 7.0 \%$ pancreatin solution and $70 \mathrm{~g}$ fresh porcine bile. Ileal starting residue consisted of $140 \mathrm{~g}$ SIES. The "meal" for the TIM-1 consisted of $100 \mathrm{~g}$ of $5.0 \%(\mathrm{w} / \mathrm{w})$ tricaprylin oil-in-water emulsion, $90 \mathrm{~g}$ gastric electrolyte solution, $50 \mathrm{~g}$ water and $11 \mathrm{mg} \alpha$-amylase (to simulate the effect of salivary amylase). The meal was immediately fed into the gastric compartment (containing $10 \mathrm{~g}$ gastric starting residue) along with a final $50 \mathrm{~g}$ water rinse for a starting volume of $300 \mathrm{~mL}$ 
Micellar fractions were obtained from the filtrates using semi-permeable, $0.05 \mu \mathrm{m}$ pore size capillary membrane filters (Spectrum Milikros modules M80S-300-01P, Repligen, Waltham, MA, USA) located in the ileal and jejunal compartments. Intestinal secretions were added into the intestinal compartments throughout digestion at controlled rates. Duodenal secretions consisted of fresh porcine bile added at $0.50 \mathrm{~mL} / \mathrm{min}, 7.0 \%(\mathrm{w} / \mathrm{v})$ pancreatin solution at $0.25 \mathrm{~mL} / \mathrm{min}$ and SIES at $3.2 \mathrm{~mL} / \mathrm{min}$. Jejunal secretions consisted of SIES and $10 \%$ fresh porcine bile at $3.20 \mathrm{~mL} / \mathrm{min}$. Ileal secretions consisted of SIES at $3.00 \mathrm{~mL} / \mathrm{min}$. Secretion of $\mathrm{HCl}(1.00 \mathrm{M})$ into the gastric compartment followed a pre-programmed $\mathrm{pH}$ curve. In the fed state, the gastric compartment $\mathrm{pH}$ decreased from $\mathrm{pH} 6.5$ to 1.7 as shown in Table 4.1. Secretion of sodium bicarbonate solution $(1.00 \mathrm{M})$ was used to maintain the $\mathrm{pH}$ of the duodenal, jejunal, and ileal compartments at $6.5,6.8$ and 7.2, respectively.

Throughout the $5 \mathrm{~h}$ simulated digestion, digestates were collected from the jejunal and ileal filtrates and at the ileal efflux at: 30, 60, 90, 120, 180, 240 and $300 \mathrm{~min}$. The collected filtrates represented the bioaccessible fraction which represents the metabolites available to be absorbed and become bioavailable. Samples collected at the ileum efflux represented what would have transited from the small intestine into the large intestine. TIM-1 experiments were performed in triplicate for each surfactant-stabilized emulsion, which were freshly prepared within $1 \mathrm{~h}$ prior to use.

\section{Extraction of FFAs}

Prior to the DCM fatty acid extraction, nonanoic acid was added as an internal standard to calculate extraction efficiency. $2.0 \mathrm{~mL}$ of digestate was adjusted to a $\mathrm{pH}$ between $10-12 \mathrm{using}$ $\mathrm{NaOH}(10 \mathrm{M})$ to deprotonate the FFAs, rendering them insoluble in DCM. Samples were then extracted with $6.0 \mathrm{~mL}$ DCM for $24 \mathrm{~h}$ at ambient temperature $\left(22-25^{\circ} \mathrm{C}\right)$. The organic DCM layer 
containing TAGs was discarded after partitioning. The aqueous layer was adjusted to $\mathrm{pH} 1-2 \mathrm{using}$ $\mathrm{HCl}(1.00 \mathrm{M})$ to protonate the FFAs, making them soluble in DCM. FFAs were extracted from the sample using $4.0 \mathrm{~mL}$ of DCM for $24 \mathrm{~h}$ at ambient temperature $\left(22-25^{\circ} \mathrm{C}\right)$. After separation, the aqueous layer containing proteins and carbohydrates was discarded. $1.0 \mathrm{~mL}$ of the DCM layer was transferred into new vials and nitrogen-dried (ZipVap Evaporator, Glas-Col, Terre Haute, IN, USA). They were then reconstituted in $1.0 \mathrm{~mL}$ acetonitrile-water (60:40) for analysis using highperformance liquid chromatography coupled with a diode-array detector (HPLC-DAD) (Agilent 1100 HPLC G1315B DAD, Santa Clara, CA, USA).

\section{HPLC-DAD Analysis}

The degree of lipolysis was determined by measuring the concentration of caprylic acid in the digestates using HPLC-DAD and a reversed-phase C18 column (Zorbax 300SB-C18, Agilent, Santa Clara, CA, USA) equipped with a guard column (Zorbax 300SB-C18, 4.6 x 12.5 mm, 5micron, Agilent, Santa Clara, CA, USA) measuring absorbance at $210 \mathrm{~nm}$, the wavelength which is absorbed via carboxyl functional groups. The column temperature was maintained at $30 \pm 5{ }^{\circ} \mathrm{C}$ and the injection volume was $10 \mathrm{uL}$. Peak resolution was obtained using a $1.0 \mathrm{~mL} / \mathrm{min}$ isocratic flow rate consisting of $60 / 40 \%(\mathrm{v} / \mathrm{v})$ acetonitrile/water with $0.1 \%$ trifluoroacetic acid (v/v). The retention time of caprylic acid and nonanoic acid was $\sim 2.7$ and $\sim 3.3 \mathrm{~min}$, respectively. Calibration standards for caprylic acid and nonanoic acid were prepared in acetonitrile-water (60:40) at the following concentrations: 78, 156, 312, 625, 1250, 2500, 5000 and $10000 \mathrm{mg} / \mathrm{L}$. Calibration curves were created by plotting peak area against corresponding concentrations and fitted using linear regression to obtain linear equations for caprylic acid $\left(y=0.0872 x-0.8506, \mathrm{R}^{2}=0.999\right)$ and nonanoic acid $\left(\mathrm{y}=0.0770 \mathrm{x}-0.9143, \mathrm{R}^{2}=0.999\right)($ Figure $S 3)$. Peak areas were integrated based on cardinal points (i.e., baseline points, valley points, peak apex, points at peak half height) using 
Agilent ChemStation for LC data-processing software (rev. C.01.07, Santa Clara, CA, USA), which establishes the baseline using trapezoidal area and proportional height corrections.

\section{Mathematical Modeling}

The generation of FFAs by lipolysis followed a sigmoidal pattern under simulated GI conditions, which has been previously reported (Li and McClements 2011; Troncoso et al. 2012; Speranza et al. 2013; Fondaco et al. 2015; Yao et al. 2018; Dias et al. 2019). The cumulative bioaccessible fraction of FFAs throughout lipolysis is characterized by a lag period of minimal lipolysis, followed by a rapid increase in FFA release and finishes with a plateauing region of cumulative FFA concentration ( $\mathrm{Li}$ and McClements 2011). The lag period is attributed to the time to displace surfactants from the oil-water interface that act as a barrier to lipase. The delay in lipase adsorption results in less FFA released due to the time required for bile to displace surfactants from the interface (Reis et al. 2009; Troncoso et al. 2012).

A three-parameter shifted logistic equation (eqn. 1) has been used to model lipolytic generation of FFAs as a function of time (t) (Speranza et al. 2013; Ye et al. 2013; Fondaco et al. 2015; Verkempinck et al. 2018):

$$
C(t)=\frac{C_{a s y m p}}{1+e^{\left[k\left(t_{c}-t\right)\right]}}-\frac{C_{a s y m p}}{1+e^{\left(k t_{c}\right)}}
$$

$\mathrm{C}_{\text {asymp }}$ is the asymptotic plateau and represents the total bioaccessible fraction of FFAs released, $\mathrm{k}$ is the rate constant of FFA release per unit time, and $t_{c}$ is the time to reach effective lipolysis at which half the total amount of FFAs are released, crudely defined here as induction time. To force the shifted logistic model to pass through the origin at $t=0$ min, since no digestion has occurred at this point in time, the second term is incorporated (Speranza et al. 2013). The cumulative FFA concentration in the jejunum and ileal filtrates were fitted using non-linear regression using leastsquares method in Graphpad Prism (version 6.0, La Jolla, CA, USA). Initial values (i.e., $\mathrm{C}_{\text {asymp }}=$ 
$\left.60 \% ; \mathrm{k}=0.02 ; \mathrm{t}_{\mathrm{c}}=100\right)$ were provided allowing the software to perform 1000 iterations to fit the data. The coefficients of determination $\left(\mathrm{R}^{2}\right)$ were all greater than 0.97 .

\section{Statistical Analysis}

TIM-1 measurements were performed in triplicate and reported as means \pm standard deviation. The total area under the curve (AUC) of absolute \% bioaccessibility of caprylic acid over the $5 \mathrm{~h}$ digestion was calculated using the trapezoidal integration method (Graphpad Prism version 6.0, La Jolla, CA, USA). As AUC results were obtained from the averaged triplicates, statistical analysis was not able to be performed. Statistically significant differences in the three parameters obtained from the shifted logistic model (i.e., $t_{c}, k$, and $C_{\text {asymp }}$ ) were determined by comparing overlaps between their 95\% confidence intervals (Graphpad Prism version 6.0, La Jolla, CA, USA). Sample parameters that did not have overlapping 95\% confidence intervals were considered statistically different.

\subsection{Results \& Discussion}

Surfactant concentration was selected to ensure consistent particle size and stability over a 7-day period (Figure $4.3,4.4$ ). The average particle sizes of o/w emulsions were measured as a function of surfactant concentration between $0-2.0 \%(w / w)$ for the synthetic surfactants, $0-1.5 \%$ (w/w) for commercial MAGs, and $0-1.0 \%(w / w)$ for 2-GMO (narrower concentration range due to limited amount of purified material). Based on the concentration as a function of average particle size (Figure 4.3), 0.5\% (w/w) surfactant was maintained constant across all samples, except for $2-$ GMO which was at $0.7 \%(\mathrm{w} / \mathrm{w})$ because emulsion stability was not achieved at $0.5 \%(\mathrm{w} / \mathrm{w})$. These values were selected as they were the lowest concentration to achieve the desired average particle size (i.e., $230 \pm 30 \mathrm{~nm}$ ) with monolayer coverage based on our emulsification protocol. The synthetic surfactants (Spans and Tweens) and commercial monoglycerides (1-GMO and 1-GMS) 
were expected to be more effective surfactants and better at reducing particle size compared to 2GMO, due to symmetry of the attached hydroxyl groups for $s n-2$ MAG molecules (Lauridsen 1976; Krog 1977). To confirm the chosen concentrations were able to produce stable emulsions, particle size distributions were measured over 7 days (Figure 4.4). Emulsions were stable at the chosen concentrations for each surfactant as particle size distributions remained monomodal with no shift in peak position over the 7-day period.

Bioaccessibility is reported as the percentage of caprylic acid hydrolyzed from tricaprylin by porcine pancreatic lipase collected in the jejunal and ileal filtrates (Figure 4.5). Irrespective of the surfactant used to stabilize the o/w emulsions, the absolute bioaccessible fraction peaked at $\sim 180 \mathrm{~min}$ and then progressively declined for the remaining $120 \mathrm{~min}$ (Figure 4.5). The cumulative bioaccessible fraction as a function of time was fitted using the three-parameter shifted logistic model (eqn. 1) (Figure 4.6). The parameters obtained (i.e., $\mathrm{t}_{\mathrm{c}}, \mathrm{k}$, and $\mathrm{C}_{\text {asymp }}$ ) from the shifted logistic model were compared to study induction times, lipolysis kinetics and FFA bioaccessibility throughout the simulated digestion period (Table 4.3).

The time to reach effective lipolysis $\left(t_{c}\right)$ obtained from the cumulative bioaccessibility curves (Figure 4.6) were interpreted as an indirect measure of induction time and were not statistically different in the jejunum (Figure 4.7). A previous study by Speranza et al. (2013) observed significant differences in induction time in the jejunum; however, a considerably higher surfactant concentration (1.5\% w/w compared to $0.5-0.7 \% \mathrm{w} / \mathrm{w})$ was used (Speranza et al. 2013). This study used the minimal surfactant concentration $(0.5-0.7 \% \mathrm{w} / \mathrm{w})$ required to achieve desired average particle size (i.e., $230 \pm 30 \mathrm{~nm}$ ) and maintain monomodal particle size distributions over a 7-day period (Figure 4.3, 4.4). An initial lag period observed before effective lipolysis has been previously attributed to an interfacial surfactant barrier that delays lipase accessibility to lipid 
droplet interface (Reis et al. 2009; Troncoso et al. 2012). Due to the frequency of our sampling interval and the pre-programmed transit time set for the TIM-1, which controls gastric emptying, significant changes in induction time were not observed (Figure 4.7).

Lipolysis rate constants $(\mathrm{k})$ for the $\mathrm{o} / \mathrm{w}$ emulsions stabilized with various surfactants were not statistically different in either the jejunal or ileal filtrates (Figure 4.8). Transit of the emulsion through simulated GI conditions causes physicochemical changes at the interface due to the generation of surface-active metabolites; by the time the emulsion reaches the ileal compartment, the original surfactant monolayer is displaced by bile salts and other surface-active lipolytic products (i.e., FFAs, MAGs) (Speranza et al. 2013). There were no significant differences in rates of lipolysis amongst o/w emulsions stabilized by non-ionic, synthetic surfactants (Figure 4.8). Studies have shown Span-stabilized emulsions are acid-unstable, while Tween-stabilized emulsions are acid-stable under gastric conditions (Marciani et al. 2006, 2007, 2009). A study by Verkempinck et al. (2018) compared in vitro lipolysis of emulsions stabilized by Tween 80 and sucrose esters $(0.5 \% \mathrm{w} / \mathrm{v})$ and demonstrated that the acid-instability of sucrose esters led to coalescence in the gastric conditions (Verkempinck et al. 2018). Previously, it has been shown that oil droplet size determines the interfacial surface area available for enzyme absorption. The reaction rate constant was highest for Tween 80 -stabilized emulsions due to smaller oil droplet sizes $(1.37-1.50 \mu \mathrm{m})$ at the beginning of the intestinal phase compared to sucrose ester-stabilized emulsions (40.84-77.58 $\mu \mathrm{m})$ (Verkempinck et al. 2018).

An in vivo study by Marciani et al. (2006) reported the half-life of gastric emptying differed between acid-stable Tween 60 (107 \pm 11 min) compared to acid-unstable Span 80 (84 \pm 14 min) o/w emulsions (Marciani et al. 2006). In the stomach, phase separation of acid-unstable emulsions into aqueous and lipid layers caused the aqueous layer to empty the stomach more rapidly than the 
acid-stable meals, resulting in slower gastric emptying for acid-stable emulsions (Marciani et al. 2006, 2007, 2009). As acid-stable emulsions provided larger surface areas during lipolysis, greater concentrations of FFAs were able to reach the duodenum and trigger the duodenal brake mediated by release of cholecystokinin, which reduced the gastric emptying rate (Marciani et al. 2009). A limitation of the TIM-1 is that the instrument controls gastric emptying rates (i.e., fed state protocol gastric emptying half-time $=80 \mathrm{~min}$ ) and does not vary between emulsions. This suggests that differences in acid stability between the non-ionic, synthetic surfactants had a limited role in modifying lipolysis rates due to regulated gastric emptying of the in vitro TIM-1 system and lack of hormonal feedback mechanisms (i.e., duodenal brake).

Similarly, there were no significant differences in lipolysis rates in the jejunum and ileum for the monoglyceride-stabilized o/w emulsions (Figure 4.8). Previous work by Reis and coauthors (2008) used the TIM-1 to compare the influence of $s n-2$ monopalmitin, $\beta$-lactoglobulin and lysophosphatidylcholine on in vitro lipid digestion of tricaprylin o/w emulsions. They reported that the presence of $s n-2$ monopalmitin had an inhibitory effect on lipolysis based on the reduction in concentration of caprylic acid measured in TIM-1 intestinal compartments (i.e., duodenal, jejunal, ileal, and ileal efflux) throughout the simulated digestion period (Reis et al. 2008b). Rate constant values used here to assess lipolysis kinetics were obtained from the cumulative bioaccessible fractions as a function of time fitted using the shifted logistic model (eqn. 1) from the jejunal and ileal filtrates. Using this shifted logistic model, significant differences in rate constant values were previously demonstrated at higher concentrations of surfactants $(1.5 \% \mathrm{w} / \mathrm{w})$ for o/w emulsions in jejunal filtrates, while no differences were observed in ileal filtrates (Speranza et al. 2013). Therefore, at lower concentrations $(0.5-0.7 \% \mathrm{w} / \mathrm{w})$ of surfactants used, significant 
differences in rate constant values may only be observable prior to the emulsions reaching the jejunal compartment.

AUC values obtained from the absolute bioaccessible lipid fractions over the $5 \mathrm{~h}$ digestion period (Figure 4.5) showed o/w emulsions stabilized by Span 80 and 2-GMO resulted in lower AUC bioaccessibility values compared to the other surfactants (Figure S4). It is important to note the TIM-1 digestion model does not account for first-pass metabolism in the liver; AUC measurements are typically proportional to the fraction of absorbed compound assuming the rate of elimination from the body follows linear kinetics (i.e., clearance is constant and concentration is uniform) (Rescigno 2000). Overall bioaccessibility (Figure 4.6) was interpreted as the plateau in the cumulative bioaccessible fraction of caprylic acid (i.e., $\mathrm{C}_{\text {asymp}}$ ) using the three-parameter shifted logistic model (eqn. 1). Similar to the AUC results, overall bioaccessibility was significantly lower in both the jejunum and ileum for Span 80- and 2-GMO-stabilized o/w emulsions (Figure 4.9). Trends for AUC and overall bioaccessibility, obtained from the shifted logistic model, were consistent: Span 80- and 2-GMO-stabilized o/w emulsions had lower overall bioaccessibility in both the jejunum and ileum compared to o/w emulsions stabilized by the other surfactants (Figure S4, 4.9).

Span 80-stabilized o/w emulsions resulted in significantly lower overall bioaccessibility in the jejunum and ileum compared to o/w emulsions stabilized by the other non-ionic, synthetic surfactants (Figure 4.9). According to Cornec and co-authors (1996), the small hydrophilic head group relative to the length of the fatty acid chain of Span 80 is much less effective at protein displacement compared to the larger hydrophilic head groups on water-soluble surfactants (i.e., Tweens) (Cornec et al. 1996). The sorbitan head group of Span 80 has high head group motility (measured via dielectric dispersion analysis) that allows it to impart flexibility and fluidity at 
interfacial surfaces (Hayashi et al. 2011). A study by Peltonen et al. (2001) compared sorbitan monolayers using surface compressional moduli calculated from surface pressure-molecular area isotherms, based on known values for liquid-expanded monolayers $(12.5-50 \mathrm{mN} / \mathrm{m})$ and liquidcondensed layers (100-250 mN/m) (Davies and Rideal 1963; Peltonen et al. 2001). Although Span 60 consists of the same aliphatic chain length as Span 80, the double bond in the oleic acid chain of Span 80 imparts a dominant bent conformation. As a result, Span 60 formed the most condensed and stable monolayer at physiological temperature, attributed to its saturated hydrocarbon chain that promotes stronger chain-chain interactions; while Span 80 formed expanded monolayers (Peltonen et al. 2001). Therefore, the significantly lower overall bioaccessibility observed for Span 80-stabilized compared to Span 60-stabilized emulsions may be attributed to differences in interfacial monolayer structure. Monolayer properties of emulsion droplets affect emulsion stability by altering coalescence and flocculation (Patino and Domínguez 1993). The structure and stability of interfacial films are influenced by packing of surfactants at the interface: molecular association is stronger in condensed monolayers due to closer packing, promoting greater emulsion stability against coalescence; in comparison, expanded monolayers that have weaker mechanical surface properties and less intermolecular interactions result in less stable emulsions (Dickinson et al. 1988; Patino and Domínguez 1993). As lipases are present in excess in the GI tract, larger available interfacial surface area allows for more lipase molecules to absorb to the oil-water interface (Armand et al. 1999). Emulsion destabilization (i.e., droplet coalescence) during digestion decreases available interfacial area for lipolytic activity, leading to a lower extent of lipolysis (Salvia-Trujillo et al. 2013, 2017; Day et al. 2014; Zhang and McClements 2016; Verkempinck et al. 2018). 
An accumulation of surface-active lipolytic products at the oil-water interface occurs when the rate of hydrolysis exceeds their rate of incorporation into mixed bile salt micelles (Carey et al. 1983; Reis et al. 2010). The surface-activity of lipolytic products is greatest for 2-MAGs, followed by FFAs, DAGs and TAGs (Reis et al. 2008b; Golding and Wooster 2010). Pancreatic lipases exhibit their greatest activity on TAGs, followed by DAGs and MAGs (Senior and Isselbacher 1963). Emulsions stabilized with 2-GMO resulted in lower AUC values and overall bioaccessibility compared to emulsions stabilized with 1-GMO and 1-GMS (Figure S4, 4.9). This agrees with the previously mentioned study by Reis et al. (2008) that reported emulsions stabilized with $s n-2$ monopalmitin largely reduced the amount of FFAs generated throughout the in vitro digestion period (Reis et al. 2008b). A combination of tensiometry, ellipsometry and interfacialrheological studies confirmed 2-MAGs (i.e., sn-2 monocaprylin and sn-2 monopalmitin) are highly surface-active and can displace $s n-1,3$ regiospecific lipases from the oil-water interface (Reis et al. 2008a). Commercial monoglycerides typically contain $95 \% s n-1 / 3$ isomers and $5 \% s n-2$ isomers (Krog and Sparso 2004). As pancreatic lipases cleave sn-1/3 ester bonds, commercial-grade 1GMS and 1-GMO were potentially cleaved by pancreatic lipase (Reis et al. 2009). On the other hand, an interfacial accumulation of 2-MAGs which cannot be cleaved by pancreatic lipase would be expected to impact the total extent of lipolysis (Reis et al. 2008a). Overall, this suggests the significantly lower overall bioaccessibility observed with 2-GMO-stabilized emulsions was due to an accumulation of 2-GMO at the interface hindering lipase from accessing the substrate (Figure S4, 4.9). 


\subsection{Conclusions}

The objective of this study was to investigate the effects of $s n$-position for monoglycerides and non-ionic, synthetic surfactants on lipid digestion of o/w emulsions using the TIM-1 simulated digestion model. Significant changes in induction time were not observed due to the frequency of the sampling intervals and pre-programmed transit time set for the TIM-1 system. The kinetics of lipid digestion were not significantly different in the jejunum and ileum due to the generation of surface-active metabolites in situ and surfactant concentrations used $(0.5-0.7 \% \mathrm{w} / \mathrm{w})$. Acid stability of surfactants played a minimal role in modifying lipolysis rates due to the preprogrammed gastric emptying of the TIM-1 system and lack of hormonal feedback mechanisms. Both Span 80- and 2-GMO-stabilized o/w emulsions had statistically lower overall bioaccessibility compared to o/w emulsions stabilized by other surfactants tested. For Span 80-stabilized emulsions, lower bioaccessibility was attributed to the unsaturated oleic acid chain forming less stable expanded monolayers; thus, reducing emulsion stability during digestion thereby decreasing the available interfacial area for lipolytic activity (Dickinson et al. 1988; Patino and Domínguez 1993). For 2-GMO-stabilized emulsions, the reduced bioaccessibility was attributed to an accumulation of 2-GMO at the interface, which hindered lipase adsorption and activity. The extent of lipolysis was expected to be decreased for an o/w emulsion system consisting of an interface covered by $s n-2$ monoglycerides, which are resistant to enzymatic hydrolysis by $1 / 3$-regiospecific pancreatic lipase (Reis et al. 2008b, 2010). Trends for AUC and overall bioaccessibility, obtained from the shifted logistic model, were consistent. These results confirm that sn-position of monoglycerides and surfactant selection significantly influence the extent of lipid digestion in vitro and provides an opportunity to tailor lipemic index of processed foods. 


\subsection{References}

Abrams CK, Hamosh M, Dutta SK, et al (1987) Role of nonpancreatic lipolytic activity in exocrine pancreatic insufficiency. Gastroenterology 92:125-129 . doi: 10.1016/00165085(87)90848-1

Afshin A, Sur PJ, Fay KA, et al (2019) Health effects of dietary risks in 195 countries, 19902017: a systematic analysis for the Global Burden of Disease Study 2017. Lancet. doi: 10.1016/S0140-6736(19)30041-8

Armand M, Pasquier B, André M, et al (1999) Digestion and absorption of 2 fat emulsions with different droplet sizes in the human digestive tract. Am J Clin Nutr 70:1096-1106 . doi: 10.1093/ajen/70.6.1096

Bansal S, Buring JE, Rifai N, et al (2007) Fasting compared with nonfasting triglycerides and risk of cardiovascular events in women. J Am Med Assoc 298:309-316 . doi: 10.1001/jama.298.3.309

Baraldi LG, Martinez Steele E, Canella DS, Monteiro CA (2018) Consumption of ultraprocessed foods and associated sociodemographic factors in the USA between 2007 and 2012: Evidence from a nationally representative cross-sectional study. BMJ Open 8:e020574 . doi: 10.1136/bmjopen-2017-020574

Carey MC, Small DM, Bliss CM (1983) Lipid Digestion and Absorption. Annu Rev Physiol 45:651-677 . doi: 10.1146/annurev.ph.45.030183.003251

Cediel G, Reyes M, Da Costa Louzada ML, et al (2018) Ultra-processed foods and added sugars in the Chilean diet (2010). Public Health Nutr 21:125-133 . doi: $10.1017 / \mathrm{S} 1368980017001161$

Compton DL, Vermillion KE, Laszlo JA (2007) Acyl migration kinetics of 2-Monoacylglycerols from soybean oil via 1H NMR. JAOCS, J Am Oil Chem Soc 84:343-348 . doi: 10.1007/s11746-007-1049-1

Cornec M, Mackie AR, Wilde PJ, Clark DC (1996) Competitive adsorption of $\beta$-lactoglobulin and $\beta$-casein with Span 80 at the oil-water interface and the effect on emulsion behaviour. Colloids Surfaces A Physicochem Eng Asp 114:237-244 . doi: 10.1016/09277757(96)03538-8

Davies JT, Rideal EK (1963) Interfacial phenomena. Academic Press, London

Day L, Golding M, Xu M, et al (2014) Tailoring the digestion of structured emulsions using mixed monoglyceride-caseinate interfaces. Food Hydrocoll 36:151-161 . doi: 10.1016/j.foodhyd.2013.09.019

Dekker MJ, Wright AJ, Mazurak VC, et al (2009) Fasting triacylglycerol status, but not polyunsaturated/saturated fatty acid ratio, influences the postprandial response to a series of oral fat tolerance tests. J Nutr Biochem 20:694-704 . doi: 10.1016/j.jnutbio.2008.06.012

DeNigris SJ, Hamosh M, Kasbekar DK, et al (1988) Lingual and gastric lipases: species differences in the origin of prepancreatic digestive lipases and in the localization of gastric lipase. Biochim Biophys Acta (BBA)/Lipids Lipid Metab 959:38-45 . doi: 10.1016/00052760(88)90147-6

Dias CB, Zhu X, Thompson AK, et al (2019) Effect of the food form and structure on lipid digestion and postprandial lipaemic response. Food Funct 10:112-124 . doi: $10.1039 / \mathrm{c} 8$ fo01698d

Dickinson E, Murray BS, Stainsby G (1988) Coalescence stability of emulsion-sized droplets at a planar oil-water interface and the relationship to protein film surface rheology. J Chem 
Soc Faraday Trans 1 Phys Chem Condens Phases 84:871-883 . doi: 10.1039/F19888400871

Dickinson PA, Abu Rmaileh R, Ashworth L, et al (2012) An Investigation into the Utility of a Multi-compartmental, Dynamic, System of the Upper Gastrointestinal Tract to Support Formulation Development and Establish Bioequivalence of Poorly Soluble Drugs. AAPS J 14:196-205 . doi: 10.1208/s12248-012-9333-x

Fondaco D, AlHasawi F, Lan Y, et al (2015) Biophysical Aspects of Lipid Digestion in Human Breast Milk and Similac ${ }^{\mathrm{TM}}$ Infant Formulas. Food Biophys 10:282-291 . doi: 10.1007/s11483-014-9388-6

Golding M, Wooster TJ (2010) The influence of emulsion structure and stability on lipid digestion. Curr Opin Colloid Interface Sci 15:90-101 . doi: 10.1016/j.cocis.2009.11.006

Golding M, Wooster TJ, Day L, et al (2011) Impact of gastric structuring on the lipolysis of emulsified lipids. Soft Matter 7:3513 . doi: 10.1039/c0sm01227k

Guo Q, Ye A, Bellissimo N, et al (2017) Modulating fat digestion through food structure design. Prog Lipid Res 68:109-118 . doi: 10.1016/j.plipres.2017.10.001

Hamosh M (1990) Lingual and gastric lipases. Nutrition 6:421-8

Hasenhuettl G, Hartel RW (2008) Food emulsifiers and their applications, 2nd ed.

Hayashi K, Shimanouchi T, Kato K, et al (2011) Span 80 vesicles have a more fluid, flexible and "wet" surface than phospholipid liposomes. Colloids Surfaces B Biointerfaces 87:28-35 . doi: 10.1016/j.colsurfb.2011.04.029

Hur SJ, Decker EA, McClements DJ (2009) Influence of initial emulsifier type on microstructural changes occurring in emulsified lipids during in vitro digestion. Food Chem 114:253-262 . doi: 10.1016/j.foodchem.2008.09.069

Juul F, Hemmingsson E (2015) Trends in consumption of ultra-processed foods and obesity in Sweden between 1960 and 2010. Public Health Nutr 18:3096-3107 . doi: $10.1017 / \mathrm{S} 1368980015000506$

Krog N (1977) Functions of emulsifiers in food systems. J Am Oil Chem Soc 54:124-131 . doi: $10.1007 / \mathrm{BF} 02894388$

Krog NJ, Sparso FV (2004) Food emulsifiers: their chemical and physical properties. In: Food Emulsions, 4th ed. pp 45-91

Lauridsen JB (1976) Food emulsifiers: Surface activity, edibility, manufacture, composition, and application. J Am Oil Chem Soc 53:400-407 . doi: 10.1007/BF02605731

Li Y, McClements DJ (2011) Inhibition of lipase-catalyzed hydrolysis of emulsified triglyceride oils by low-molecular weight surfactants under simulated gastrointestinal conditions. Eur J Pharm Biopharm 79:423-431 . doi: 10.1016/j.ejpb.2011.03.019

Lien EL (1994) The role of fatty acid composition and positional distribution in fat absorption in infants. J Pediatr 125:S62-S68 . doi: 10.1016/S0022-3476(06)80738-9

Louzada ML da C, Baraldi LG, Steele EM, et al (2015) Consumption of ultra-processed foods and obesity in Brazilian adolescents and adults. Prev Med (Baltim) 81:9-15 . doi:

10.1016/J.YPMED.2015.07.018

Louzada ML da C, Ricardo CZ, Steele EM, et al (2018) The share of ultra-processed foods determines the overall nutritional quality of diets in Brazil. Public Health Nutr 21:94-102 . doi: $10.1017 /$ S1368980017001434

Lu Y, Hajifathalian K, Ezzati M, et al (2014) Metabolic mediators of the effects of body-mass index, overweight, and obesity on coronary heart disease and stroke: a pooled analysis of 97 prospective cohorts with 1.8 million participants. Lancet 383:970-83 . doi: 10.1016/S01406736(13)61836-X 
Marciani L, Faulks R, Wickham MSJ, et al (2009) Effect of intragastric acid stability of fat emulsions on gastric emptying, plasma lipid profile and postprandial satiety. Br J Nutr 101:919-928 . doi: 10.1017/S0007114508039986

Marciani L, Wickham M, Singh G, et al (2007) Enhancement of intragastric acid stability of a fat emulsion meal delays gastric emptying and increases cholecystokinin release and gallbladder contraction. Am J Physiol Liver Physiol 292:G1607-G1613 . doi: 10.1152/ajpgi.00452.2006

Marciani L, Wickham MSJ, Bush D, et al (2006) Magnetic resonance imaging of the behaviour of oil-in-water emulsions in the gastric lumen of man. Br J Nutr 95:331 . doi: 10.1079/BJN20051628

Marrón-Ponce JA, Sánchez-Pimienta TG, Louzada ML da C, Batis C (2018) Energy contribution of NOVA food groups and sociodemographic determinants of ultra-processed food consumption in the Mexican population. Public Health Nutr. doi: $10.1017 /$ S1368980017002129

McClements DJ (2018) The biophysics of digestion: lipids. Curr Opin Food Sci 21:1-6 . doi: 10.1016/j.cofs.2018.03.009

McClements DJ, Decker EA, Park Y (2009) Controlling lipid bioavailability through physicochemical and structural approaches. Crit Rev Food Sci Nutr 49:48-67 . doi: 10.1080/10408390701764245

McClements DJ, Decker EA, Park Y, Weiss J (2008) Designing food structure to control stability, digestion, release and absorption of lipophilic food components. Food Biophys 3:219-228 . doi: 10.1007/s11483-008-9070-y

Meynier A, Genot C (2017) Molecular and structural organization of lipids in foods: their fate during digestion and impact in nutrition. OCL 24:D202 . doi: 10.1051/oc1/2017006

Monteiro CA, Cannon G, Levy RB, et al (2019) Ultra-processed foods: what they are and how to identify them. Public Health Nutr 22:936-941 . doi: 10.1017/S1368980018003762

Monteiro CA, Cannon G, Moubarac J-C, et al (2018a) The UN Decade of Nutrition, the NOVA food classification and the trouble with ultra-processing. Public Health Nutr 21:5-17 . doi: 10.1017/S1368980017000234

Monteiro CA, Moubarac J-C, Cannon G, et al (2013) Ultra-processed products are becoming dominant in the global food system. Obes Rev 14:21-28 . doi: 10.1111/obr.12107

Monteiro CA, Moubarac J-C, Levy RB, et al (2018b) Household availability of ultra-processed foods and obesity in nineteen European countries. Public Health Nutr 21:18-26 . doi: $10.1017 /$ S1368980017001379

Moreau H, Laugier R, Gargouri Y, et al (1988) Human preduodenal lipase is entirely of gastric fundic origin. Gastroenterology 95:1221-1226 . doi: 10.1016/0016-5085(88)90354-X

Mortensen MB, Afzal S, Nordestgaard BG, Falk E (2015) The high-density lipoprotein-adjusted SCORE model worsens SCORE-based risk classification in a contemporary population of 30824 Europeans: the Copenhagen General Population Study. Eur Heart J 36:2446-2453 . doi: 10.1093/eurheartj/ehv251

Moubarac J-C, Martins APB, Claro RM, et al (2013) Consumption of ultra-processed foods and likely impact on human health. Evidence from Canada. Public Health Nutr 16:2240-8 . doi: 10.1017/S1368980012005009

Moubarac J-C, Parra DC, Cannon G, Monteiro CA (2014) Food Classification Systems Based on Food Processing: Significance and Implications for Policies and Actions: A Systematic Literature Review and Assessment. Curr Obes Rep 3:256-272 . doi: 10.1007/s13679-014- 
0092-0

Moubarac JC, Batal M, Louzada ML, et al (2017) Consumption of ultra-processed foods predicts diet quality in Canada. Appetite 108:512-520 . doi: 10.1016/j.appet.2016.11.006

$\mathrm{Mu}$ H, Høy CE (2004) The digestion of dietary triacylglycerols. Prog Lipid Res 43:105-133 . doi: 10.1016/S0163-7827(03)00050-X

Nardocci M, Leclerc B-S, Louzada M-L, et al (2019) Consumption of ultra-processed foods and obesity in Canada. Can J Public Heal 110:4-14 . doi: 10.17269/s41997-018-0130-x

Nordestgaard BG, Benn M, Schnohr P, Tybjærg-Hansen A (2007) Nonfasting triglycerides and risk of myocardial infarction, ischemic heart disease, and death in men and women. $\mathrm{J}$ Am Med Assoc 298:299-308 . doi: 10.1001/jama.298.3.299

Ooi TC, Robinson L, Graham T, et al (2011) Proposing a "lipemic index" as a nutritional and research tool. Curr Vasc Pharmacol 9:313-7

Patel A (2004) Serum triglycerides as a risk factor for cardiovascular diseases in the Asia-Pacific region. Circulation 110:2678-2686 . doi: 10.1161/01.CIR.0000145615.33955.83

Patino JM, Domínguez MR (1993) Surface properties of monoglyceride monolayers spread on aqueous glycerol solutions. Colloids Surfaces A Physicochem Eng Asp 75:217-228 . doi: 10.1016/0927-7757(93)80433-F

Peltonen L, Hirvonen J, Yliruusi J (2001) The effect of temperature on sorbitan surfactant monolayers. J Colloid Interface Sci 239:134-138 . doi: 10.1006/jcis.2001.7520

Poti JM, Braga B, Qin B (2017) Ultra-processed Food Intake and Obesity: What Really Matters for Health-Processing or Nutrient Content? Curr Obes Rep 6:420-431 . doi: $10.1007 / \mathrm{s} 13679-017-0285-4$

Rauber F, Louzada ML da C, Steele EM, et al (2018) Ultra-processed food consumption and chronic non-communicable diseases-related dietary nutrient profile in the UK (2008-2014). Nutrients 10:E587 . doi: 10.3390/nu10050587

Reis P, Holmberg K, Miller R, et al (2009) Lipase reaction at interfaces as self-limiting processes. Comptes Rendus Chim 12:163-170 . doi: 10.1016/j.crci.2008.04.018

Reis P, Holmberg K, Miller R, et al (2008a) Competition between lipases and monoglycerides at interfaces. Langmuir 24:7400-7407 . doi: 10.1021/1a800531y

Reis P, Watzke H, Leser M, et al (2010) Interfacial mechanism of lipolysis as self-regulated process. Biophys Chem 147:93-103 . doi: 10.1016/j.bpc.2010.01.005

Reis PM, Raab TW, Chuat JY, et al (2008b) Influence of surfactants on lipase fat digestion in a model gastro-intestinal system. Food Biophys 3:370-381 . doi: 10.1007/s11483-008-9091-6

Rescigno A (2000) Area under the curve and bioavailability. Pharmacol Res 42:539-540 . doi: 10.1006/phrs.2000.0719

Salvia-Trujillo L, Qian C, Martín-Belloso O, McClements DJ (2013) Influence of particle size on lipid digestion and $\beta$-carotene bioaccessibility in emulsions and nanoemulsions. Food Chem 141:1475-1480 . doi: 10.1016/j.foodchem.2013.03.050

Salvia-Trujillo L, Verkempinck SHE, Sun L, et al (2017) Lipid digestion, micelle formation and carotenoid bioaccessibility kinetics: Influence of emulsion droplet size. Food Chem 229:653-662 . doi: 10.1016/j.foodchem.2017.02.146

Sarwar N, Danesh J, Eiriksdottir G, et al (2007) Triglycerides and the Risk of Coronary Heart Disease. Circulation 115:450-458 . doi: 10.1161/circulationaha.106.637793

Senior JR, Isselbacher KJ (1963) Demonstration of an intestinal monoglyceride lipase: an enzyme with a possible role in the intracellular completion of fat digestion. J Clin Invest 42:187-95 . doi: 10.1172/JCI104705 
Singh H, Sarkar A (2011) Behaviour of protein-stabilised emulsions under various physiological conditions. Adv Colloid Interface Sci 165:47-57 . doi: 10.1016/j.cis.2011.02.001

Singh H, Ye A (2013) Structural and biochemical factors affecting the digestion of proteinstabilized emulsions. Curr Opin Colloid Interface Sci 18:360-370 . doi: 10.1016/j.cocis.2013.04.006

Singh H, Ye A, Horne D (2009) Structuring food emulsions in the gastrointestinal tract to modify lipid digestion. Prog Lipid Res 48:92-100 . doi: 10.1016/j.plipres.2008.12.001

Speranza A, Corradini MG, Hartman TG, et al (2013) Influence of emulsifier structure on lipid bioaccessibility in oil-water nanoemulsions. J Agric Food Chem 61:6505-6515 . doi: 10.1021/jf401548r

Stipanuk MH (2006) Biochemical, physiological, and molecular aspects of human nutrition, 2nd ed. Saunders Elsevier, Philadelphia, Pa Edinburgh

Talmud PJ, Hawe E, Miller GJ, Humphries SE (2002) Nonfasting apolipoprotein B and triglyceride levels as a useful predictor of coronary heart disease risk in middle-aged UK men. Arterioscler Thromb Vasc Biol 22:1918-1923 . doi: 10.1161/01.ATV.0000035521.22199.C7

Troncoso E, Aguilera JM, McClements DJ (2012) Fabrication, characterization and lipase digestibility of food-grade nanoemulsions. Food Hydrocoll 27:355-363 . doi: 10.1016/j.foodhyd.2011.10.014

Varbo A, Nordestgaard BG (2018) Nonfasting Triglycerides, Low-Density Lipoprotein Cholesterol, and Heart Failure Risk. Arterioscler Thromb Vasc Biol 38:464-472 . doi: 10.1161/ATVBAHA.117.310269

Verkempinck SHE, Salvia-Trujillo L, Moens LG, et al (2018) Emulsion stability during gastrointestinal conditions effects lipid digestion kinetics. Food Chem 246:179-191 . doi: 10.1016/j.foodchem.2017.11.001

Vinarov Z, Tcholakova S, Damyanova B, et al (2012) Effects of emulsifier charge and concentration on pancreatic lipolysis: 2. Interplay of emulsifiers and biles. Langmuir 28:12140-12150 . doi: 10.1021/la301820w

Wahbeh GT, Christie DL (2011) Basic Aspects of Digestion and Absorption. In: Pediatric Gastrointestinal and Liver Disease. W.B. Saunders, pp 10-19.e2

Wilde PJ, Chu BS (2011) Interfacial \& colloidal aspects of lipid digestion. Adv Colloid Interface Sci 165:14-22 . doi: 10.1016/j.cis.2011.02.004

Yao X, Nie K, Chen Y, et al (2018) The influence of non-ionic surfactant on lipid digestion of gum Arabic stabilized oil-in-water emulsion. Food Hydrocoll 74:78-86 . doi: 10.1016/j.foodhyd.2017.07.043

Ye A, Cui J, Zhu X, Singh H (2013) Effect of calcium on the kinetics of free fatty acid release during in vitro lipid digestion in model emulsions. Food Chem 139:681-688 . doi: 10.1016/j.foodchem.2013.02.014

Zhang R, McClements DJ (2016) Enhancing nutraceutical bioavailability by controlling the composition and structure of gastrointestinal contents: Emulsion-based delivery and excipient systems. Food Struct 10:21-36 . doi: 10.1016/j.foostr.2016.07.006 


\subsection{Tables \& Figures}

Table 4.1 TIM-1 gastric compartment $\mathrm{pH}$ values throughout the $5 \mathrm{~h}$ fed state digestion protocol. Digestion time (min)

\begin{tabular}{ll}
\hline 0 & 6.5 \\
30 & 4.2 \\
60 & 2.9 \\
120 & 2.0 \\
210 & 1.7 \\
300 & 1.7 \\
\hline
\end{tabular}


Table 4.2 Class, common names, and HLB value of surfactants (Hasenhuettl G, Hartel 2008).

\begin{tabular}{llcc}
\hline Class & Surfactant & HLB value & \\
\hline Non-ionic sorbitans & Span 80 (sorbitan monooleate) & 4.3 & Oil-soluble \\
& Span 60 (sorbitan monostearate) & 4.7 & \\
Non-ionic & Tween 80 (polyoxyethylenesorbitan & 15.0 & \\
polysorbates & $\begin{array}{l}\text { monooleate } \\
\text { Tween 60 (polyoxyethylenesorbitan }\end{array}$ & 14.9 & Water-soluble \\
& monostearate) & & \\
Monoglycerides & GMO (glycerol monooleate) & 3.8 & Oil-soluble \\
& GMS (glycerol monostearate) & 3.8 & \\
\hline
\end{tabular}


Table 4.3 Induction time $\left(\mathrm{T}_{\mathrm{c}}\right)$, rate constant $(\mathrm{K})$, and overall bioaccessibility $\left(\mathrm{C}_{\mathrm{asymp}}\right)$ parameters obtained from the shifted logistic model in the jejunum (Jej), ileum (Ile), and combined jejunum and ileum (Jej + Ile)

\begin{tabular}{llccc}
\hline Surfactant & Compartment & $\mathrm{T}_{\mathrm{c}}(\mathrm{min})$ & $\mathrm{K}\left(\mathrm{min}^{-1}\right)$ & $\mathrm{C}_{\text {asymp }}(\%)$ \\
\hline $0.5 \%$ 1-GMS & Jej & $122.1 \pm 4.9$ & $0.02728 \pm 0.00394$ & $55.05 \pm 2.45$ \\
& Ile & - & $0.02896 \pm 0.00385$ & $21.49 \pm 0.93$ \\
& Jej + Ile & - & - & $76.58 \pm 3.15$ \\
$0.5 \%$ 1-GMO & Jej & $127.3 \pm 3.7$ & $0.02763 \pm 0.00284$ & $57.23 \pm 1.81$ \\
& Ile & - & $0.02908 \pm 0.00476$ & $19.81 \pm 1.05$ \\
& Jej + Ile & - & - & $76.98 \pm 2.55$ \\
$0.7 \%$ 2-GMO & Jej & $119.4 \pm 9.3$ & $0.02398 \pm 0.00642$ & $40.78 \pm 3.87$ \\
& Ile & - & $0.02959 \pm 0.00807$ & $16.30 \pm 1.40$ \\
& Jej + Ile & - & - & $57.03 \pm 5.01$ \\
$0.5 \%$ Span 60 & Jej & $122.6 \pm 4.2$ & $0.02735 \pm 0.00337$ & $53.86 \pm 2.04$ \\
& Ile & - & $0.02993 \pm 0.00354$ & $21.64 \pm 0.81$ \\
& Jej + Ile & - & - & $75.50 \pm 2.54$ \\
$0.5 \%$ Span 80 & Jej & $119.9 \pm 9.8$ & $0.02369 \pm 0.00662$ & $41.10 \pm 4.12$ \\
& Ile & - & $0.02856 \pm 0.00912$ & $14.03 \pm 1.46$ \\
& Jej + Ile & - & - & $55.11 \pm 5.55$ \\
$0.5 \%$ Tween 60 & Jej & $124.6 \pm 3.3$ & $0.02573 \pm 0.00234$ & $56.32 \pm 1.68$ \\
& Ile & - & $0.02760 \pm 0.00410$ & $21.27 \pm 1.08$ \\
& Jej + Ile & - & - & $77.65 \pm 2.33$ \\
& Jej & $118.9 \pm 4.7$ & $0.02750 \pm 0.00393$ & $52.29 \pm 2.28$ \\
$0.5 \%$ Tween 80 & Ile & - & $0.03061 \pm 0.00669$ & $19.05 \pm 1.30$ \\
& Jej + Ile & - & - & $71.45 \pm 2.88$ \\
\hline
\end{tabular}



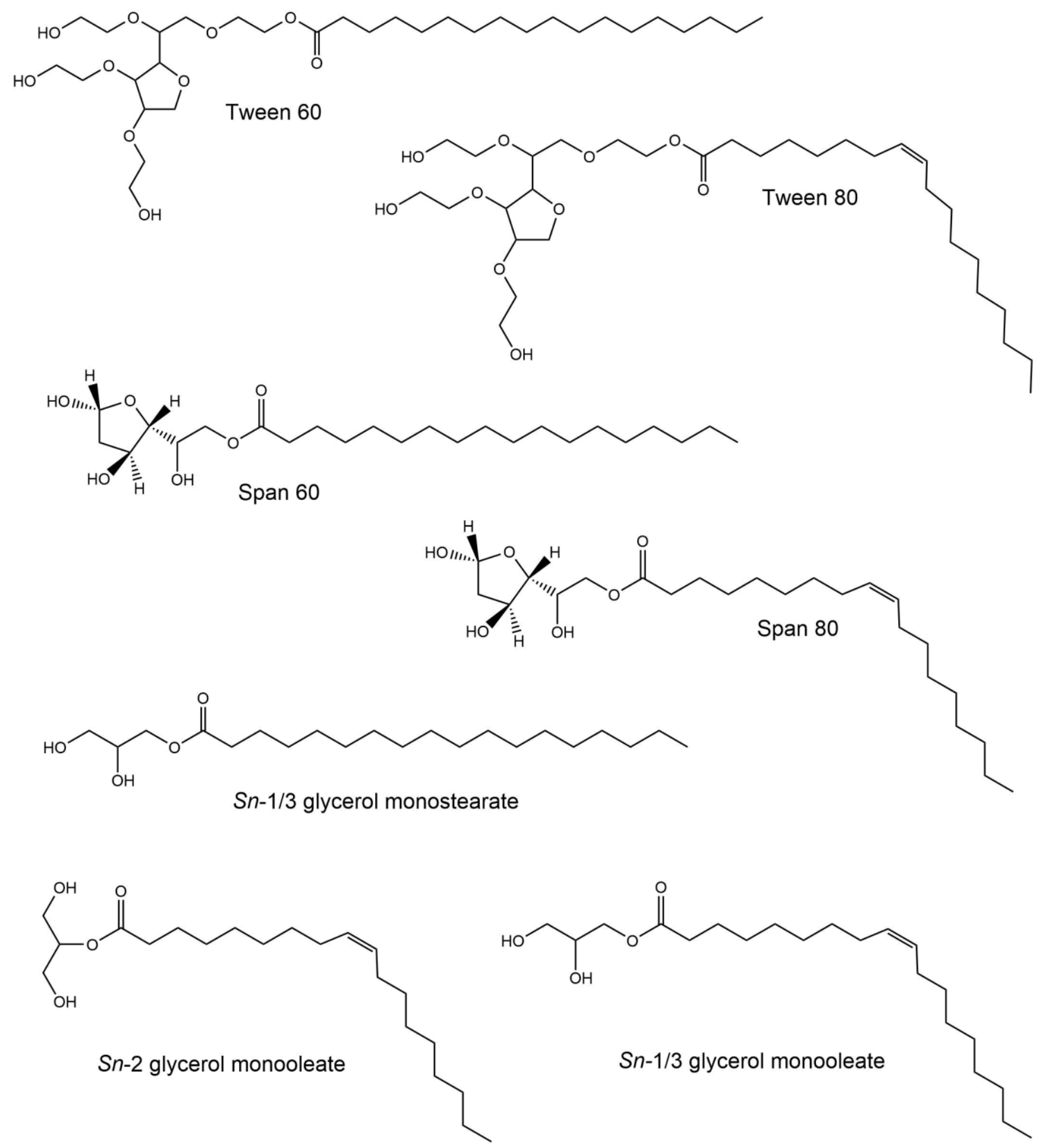

Figure 4.1 Chemical structures of surfactants employed in this study. 


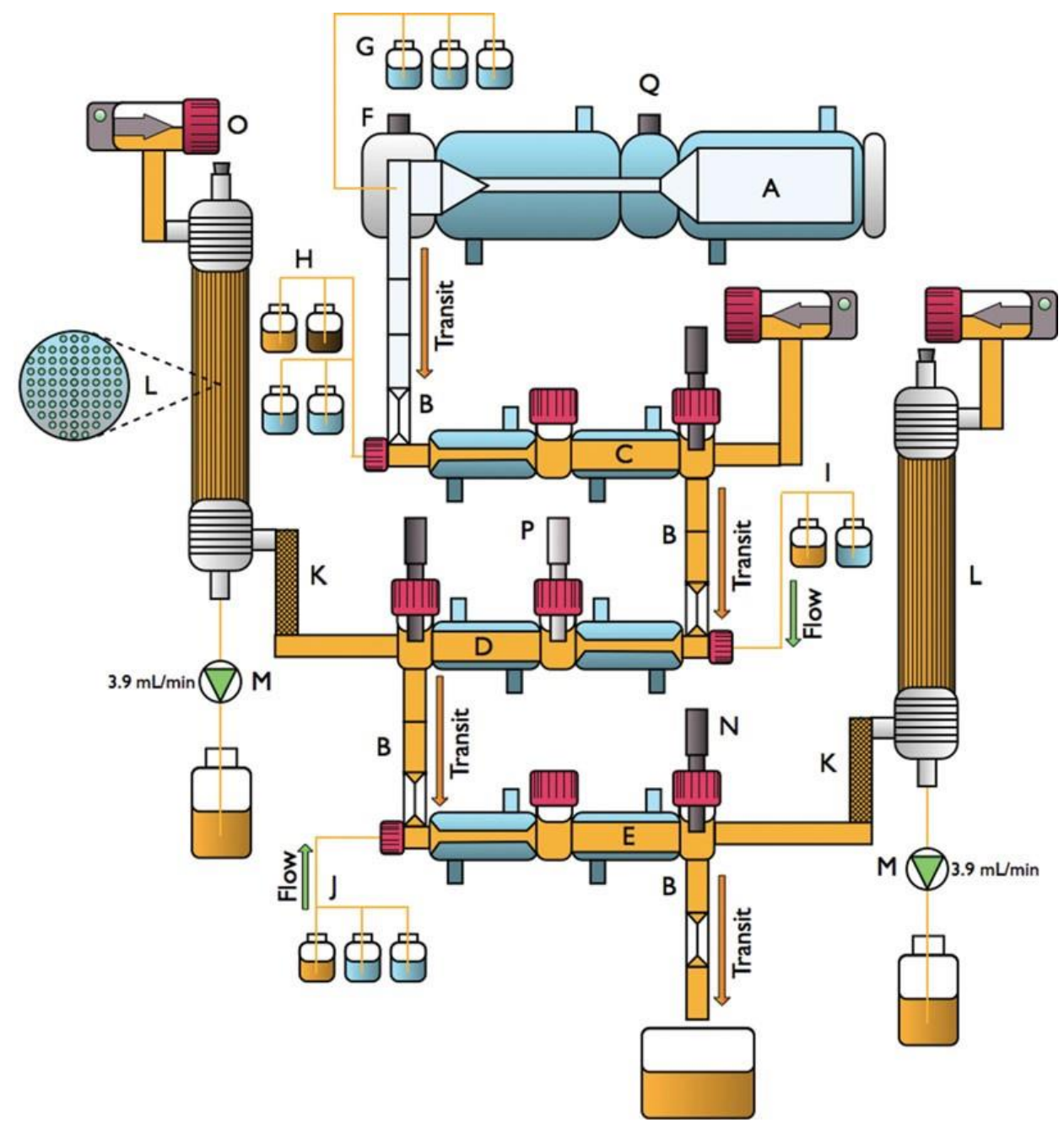

Figure 4.2 Schematic diagram of the TIM-1 system: A) gastric compartment; B) pyloric sphincter; C) duodenal compartment; D) jejunal compartment; E) ileal compartment; F) pressure sensor; G) gastric secretion; H) duodenal secretion; I) jejunal secretion; J) ileal secretion; K) pre-filter; L) semi-permeable membrane (hollow fibre cross-section); M) filtrate pump; N) pH electrode; O) level sensor; P) temperature sensor; Q) dosing port (Dickinson et al. 2012). 

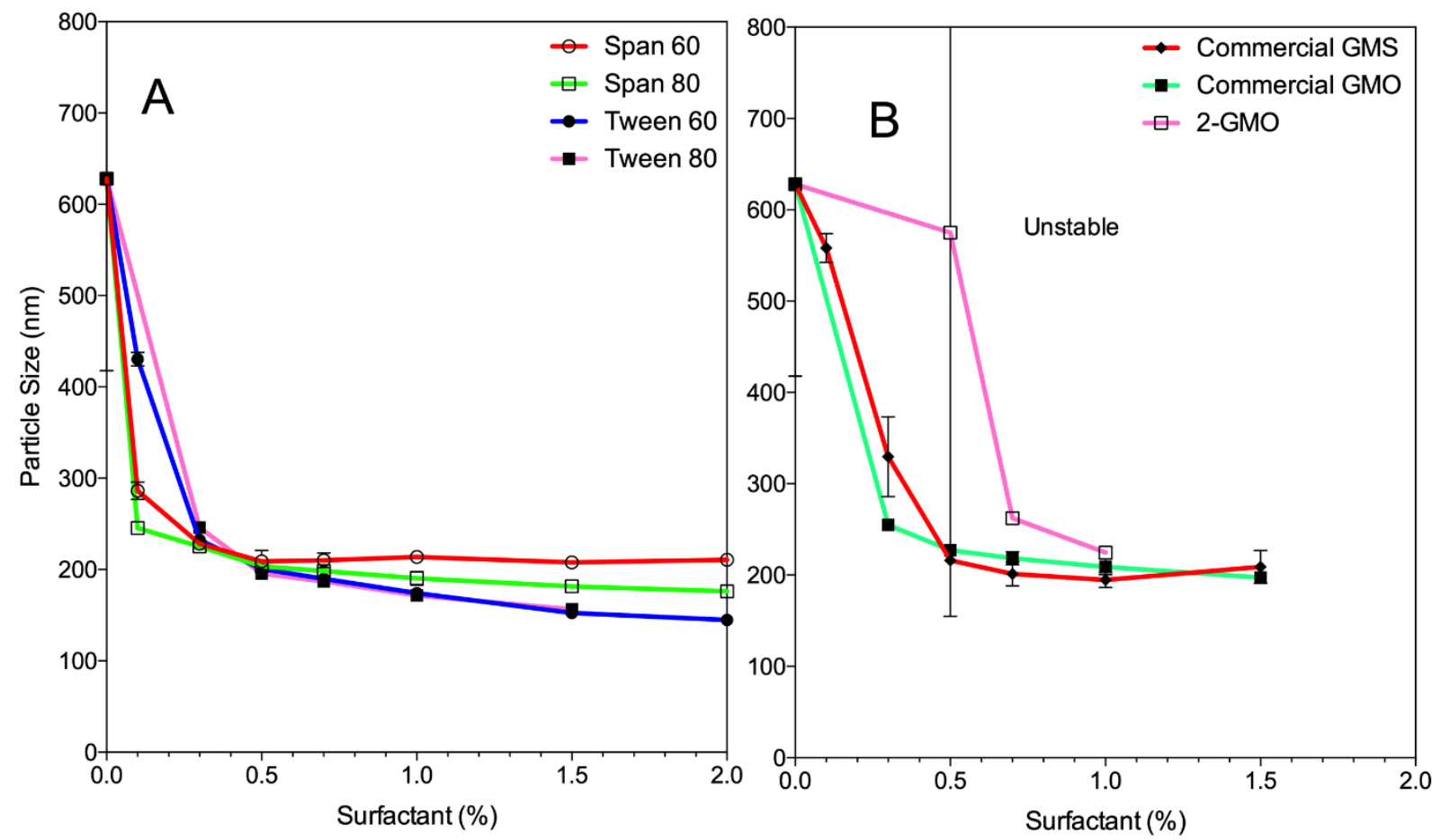

Figure 4.3 Concentration as a function of average particle size obtained for each surfactantstabilized emulsion measured; $0.5 \%(\mathrm{w} / \mathrm{w})$ was chosen as the lowest concentration at which desired particle size is achieved for all surfactants, except for $2-\mathrm{GMO}$ at $0.7 \%$ (w/w) concentration. 

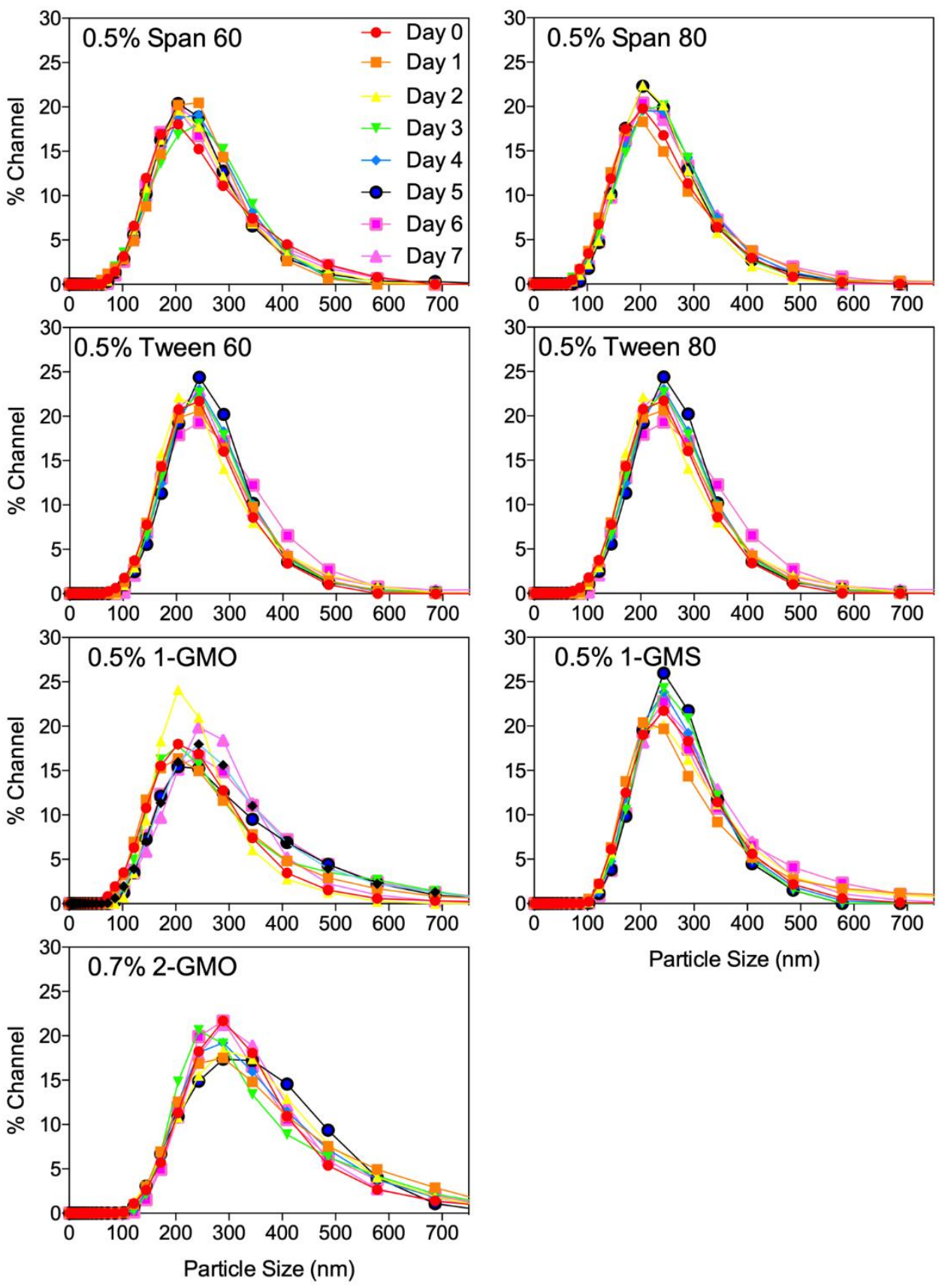

Figure 4.4 Particle size distributions of surfactant-stabilized $\mathrm{o} / \mathrm{w}$ emulsions stored at $37^{\circ} \mathrm{C}$; emulsions were considered stable when particle size distributions remained monomodal with no major shift in peak over the 7-day period. 

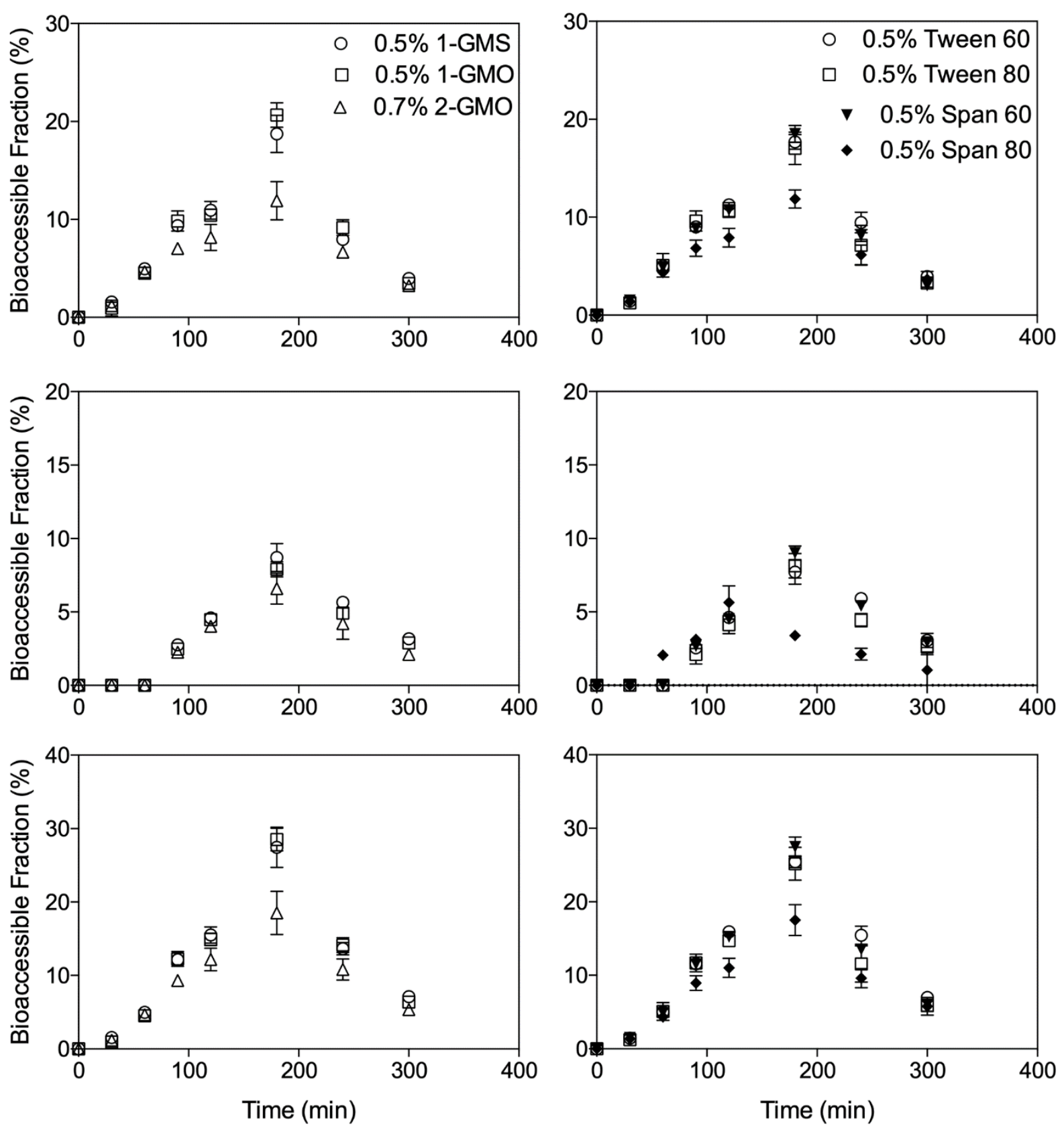

Figure 4.5 Absolute caprylic acid bioaccessibility from low molecular weight surfactant stabilized tricaprylin oil-in-water emulsions over a $5 \mathrm{~h}$ simulated digestion period. 

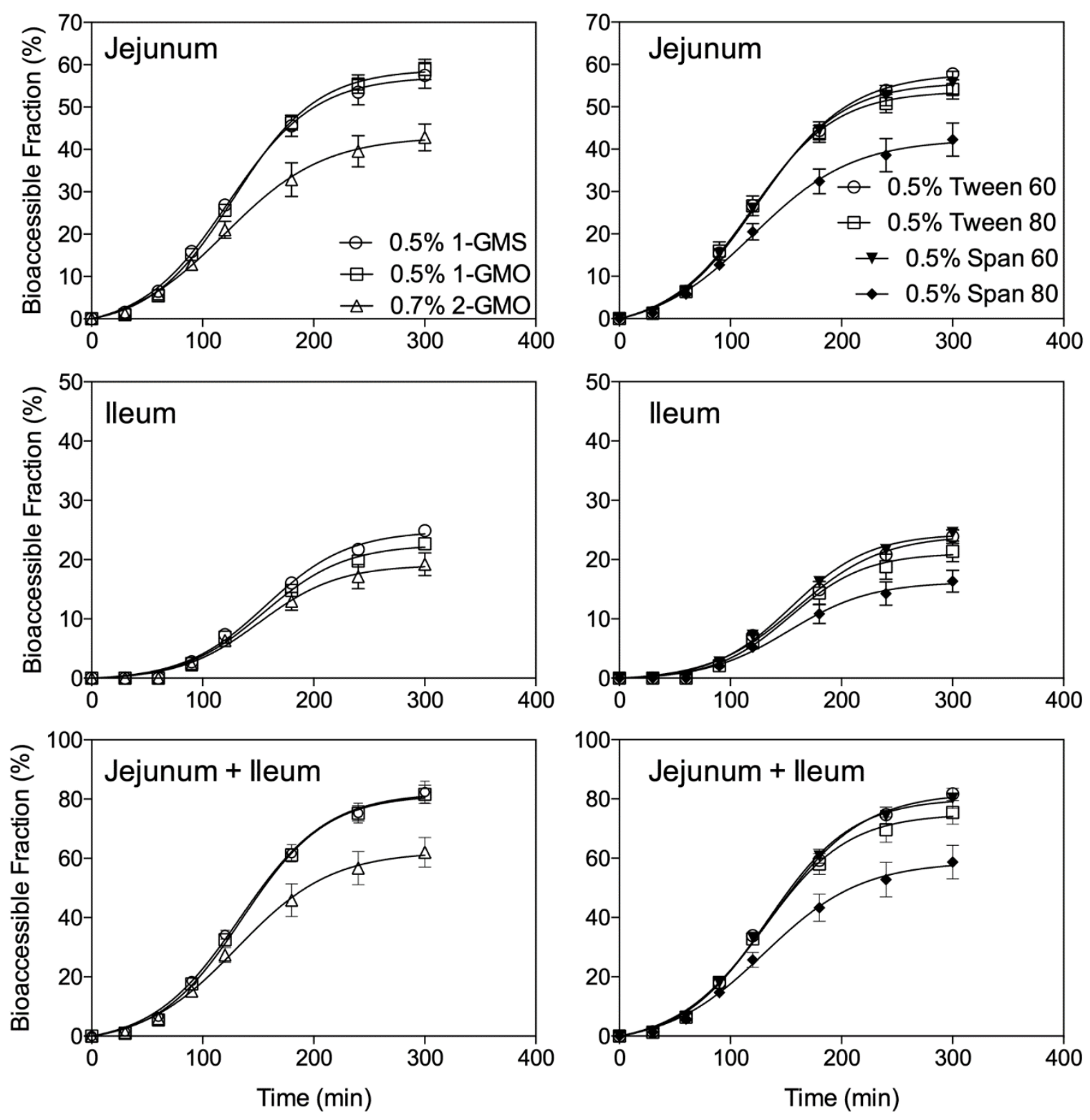

Figure 4.6 Cumulative percent bioaccessibility of caprylic acid cleaved from tricaprylin oil-inwater emulsions stabilized with various low molecular weight surfactants over a $5 \mathrm{~h}$ simulated digestion period. 


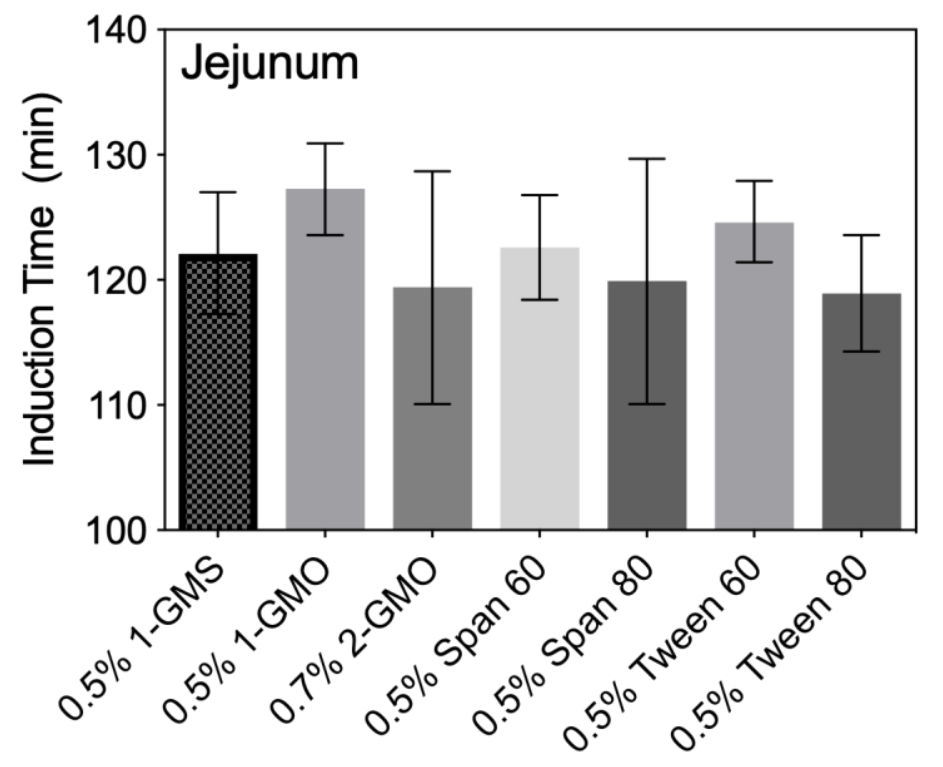

Figure 4.7 Induction time obtained from the shifted logistic model of the jejunal filtrate. No significant differences between samples were observed $(p>0.05)$. 

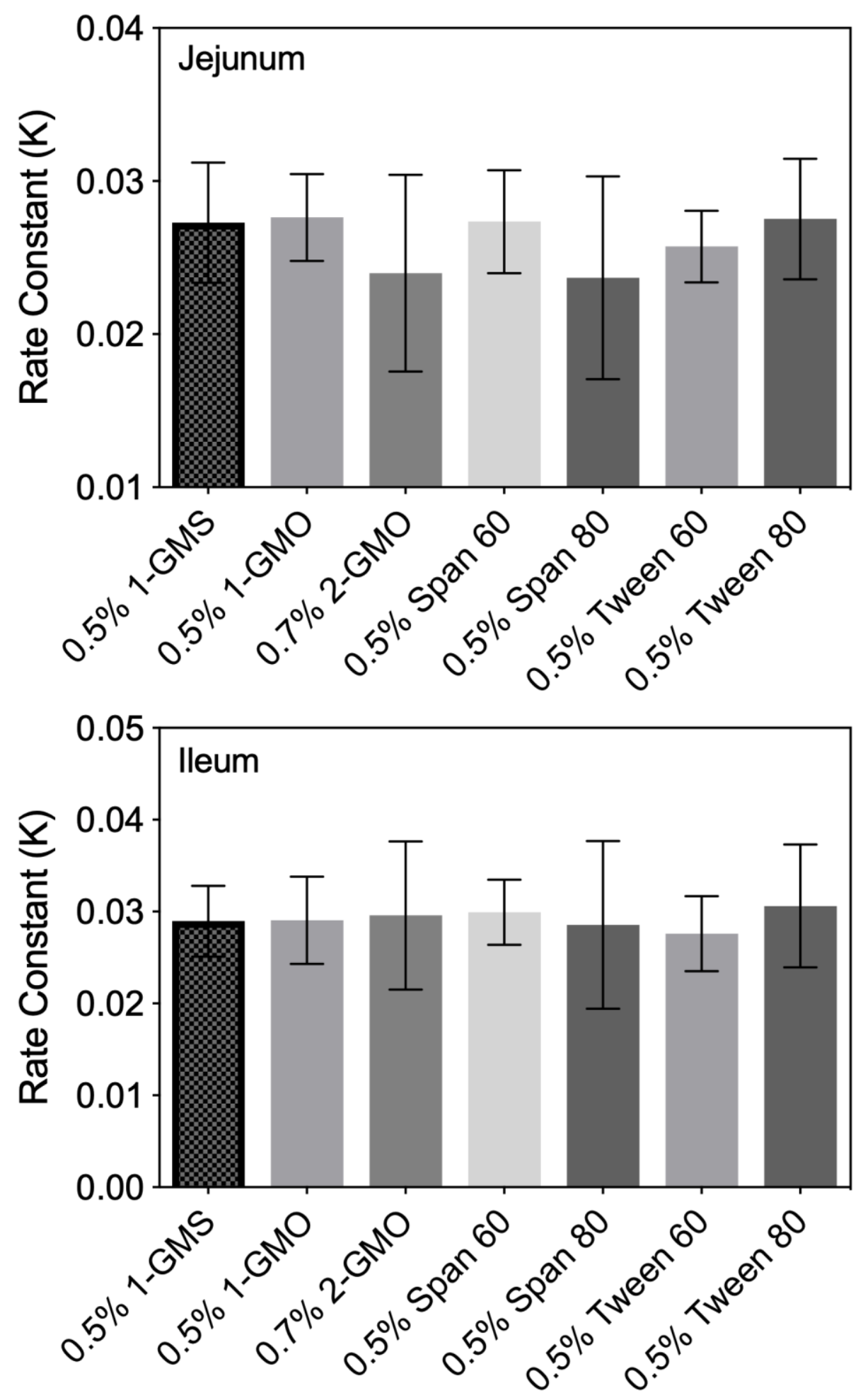

Figure 4.8 Rate constant calculated from the shifted logistic model of the jejunal and ileal filtrate. No significant differences between samples were observed $(p>0.05)$. 

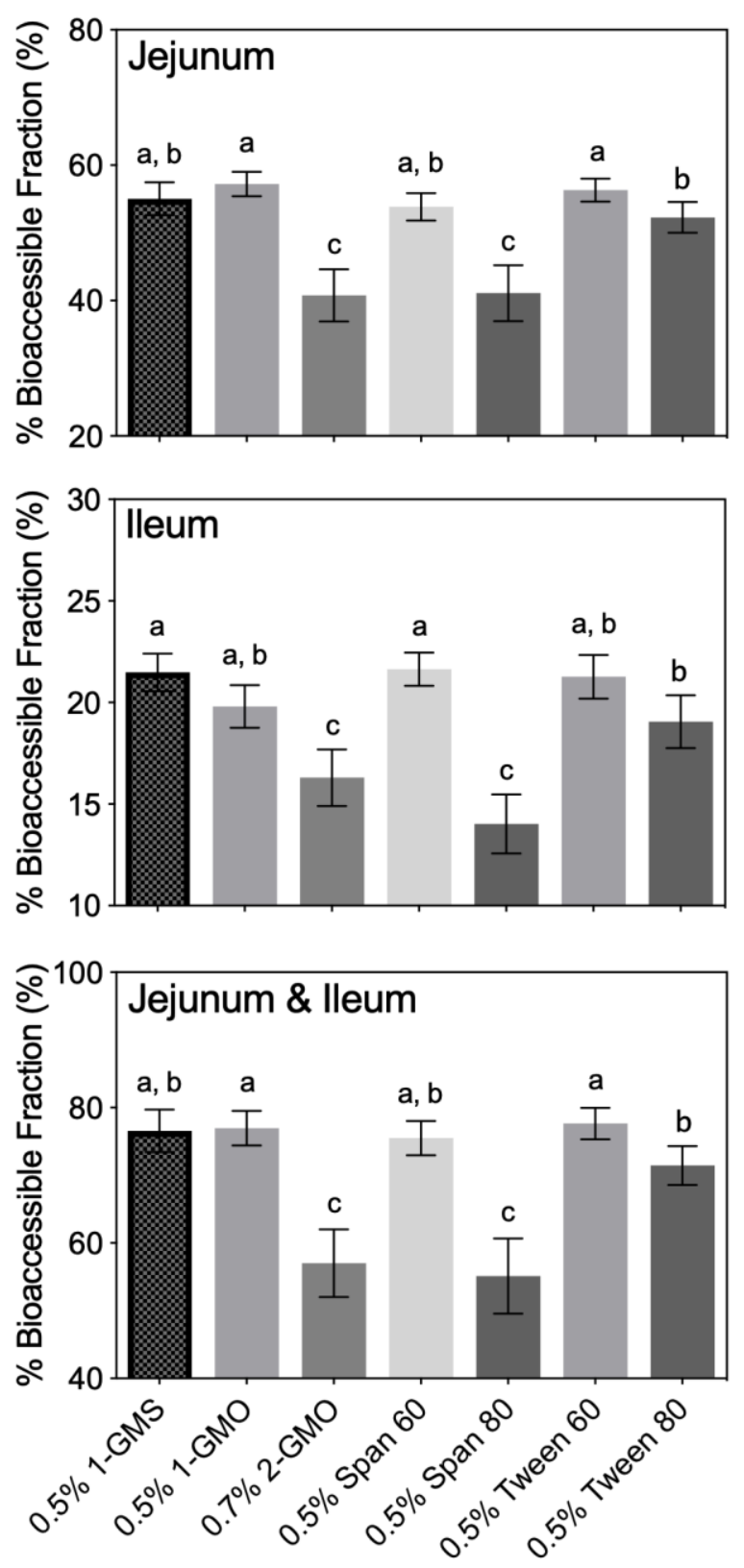

Figure 4.9 Total caprylic acid bioaccessibility (i.e., $\mathrm{C}_{\text {asymp }}$ ) calculated from the shifted logistic model of the jejunal and ileal filtrate. Different letters indicate significant differences between samples $(p<0.05)$. 


\subsection{Supporting Information}

Proton NMR

The ${ }^{1} \mathrm{H}$ NMR spectrum was collected on a Bruker AVANCE III $600 \mathrm{MHz}$ spectrometer (Bruker, Milton, ON, Canada) equipped with a $5 \mathrm{~mm}$ TCI cryoprobe (Bruker, Milton, ON, Canada). A $20 \mathrm{uL}$ sample of purified $s n-2$ glycerol monooleate (2-GMO) was dissolved in 600 uL $\mathrm{CDCl}_{3}(99.9 \%$, Cambridge Isotype Laboratories, Tewksbury, MA, USA) and temperature was regulated at $298 \pm 1 \mathrm{~K}$. The spectrum was referenced to residual internal $\mathrm{CHCl}_{3}(\delta 7.26)$. Purified 2-GMO: ${ }^{1} \mathrm{H}$ NMR $\left(\mathrm{CDCl}_{3}, 600 \mathrm{MHz}\right): \delta 5.34(\mathrm{~m}, 2 \mathrm{H}), 4.92$ (quint, $\left.1 \mathrm{H}, J=4.7 \mathrm{~Hz}\right), 3.83$ (m, 4H), $2.37(\mathrm{t}, 2 \mathrm{H}, J=7.6 \mathrm{~Hz}), 2.01(\mathrm{~m}, 4 \mathrm{H}), 1.64$ (quint, $2 \mathrm{H}, J=7.4 \mathrm{~Hz}), 1.28(\mathrm{~m}, 36.6 \mathrm{H})$, $0.88(\mathrm{t}, 12.6 \mathrm{H}, J=7.0 \mathrm{~Hz})$. The inflated integrations of $36.6 \mathrm{H}$ and $12.6 \mathrm{H}$ are due to residual heptane in the sample (Figure S1). These assignments are consistent with those reported for $s n-2$ monoglycerides from soybean oil (Compton et al. 2007).

The region from 5.25 to $3.5 \mathrm{ppm}$ of the ${ }^{1} \mathrm{H}$ NMR spectrum (Figure S2) included intense peaks attributed to glycerol backbone protons of 2-GMO and low intensity peaks corresponding to the glycerol backbone protons of diolein and $s n-1 / 3$ glycerol monooleate. Other low intensity peaks in this region included a multiplet corresponding to a $\mathrm{CH}_{2}$ group from the ethyl oleate byproduct at $4.11 \mathrm{ppm}$ and a peak at $4.02 \mathrm{ppm}$ attributed to an unknown impurity, which was also present in the ${ }^{1} \mathrm{H}$ NMR of the pure triolein starting material (data not shown). By integrating all the peaks in this region, the purity of 2-GMO was calculated relative to all the glycerolcontaining compounds and found to be $\sim 97 \mathrm{~mol} \%$. 


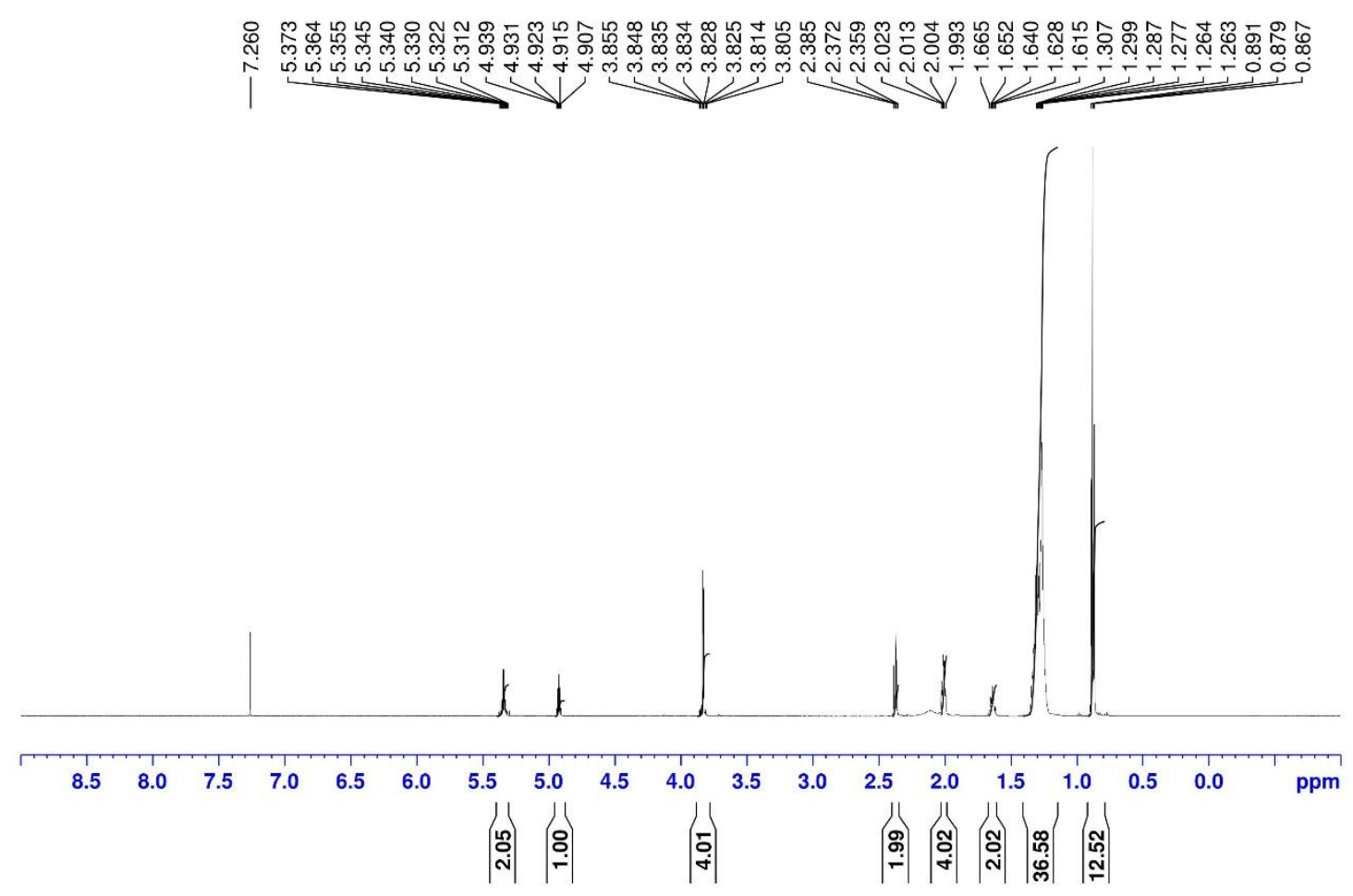

Figure S1. ${ }^{1} \mathrm{H}$ NMR spectrum $\left(\mathrm{CDCl}_{3}, 600 \mathrm{MHz}\right)$ of 2-GMO. 


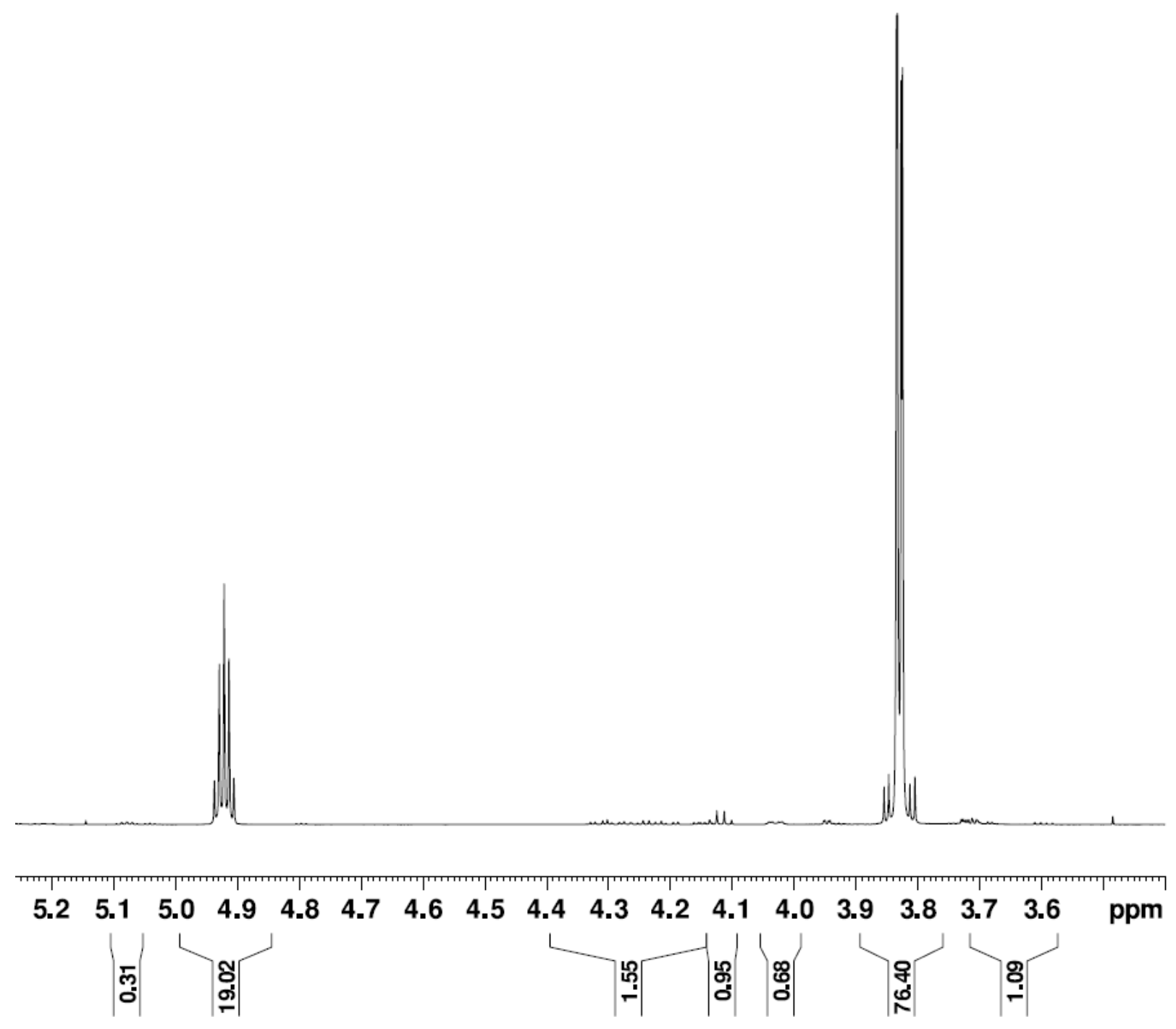

Figure S2. ${ }^{1} \mathrm{H} \mathrm{NMR}$ spectrum $\left(\mathrm{CDCl}_{3}, 600 \mathrm{MHz}\right)$ of the glycerol backbone region of 2-GMO. 


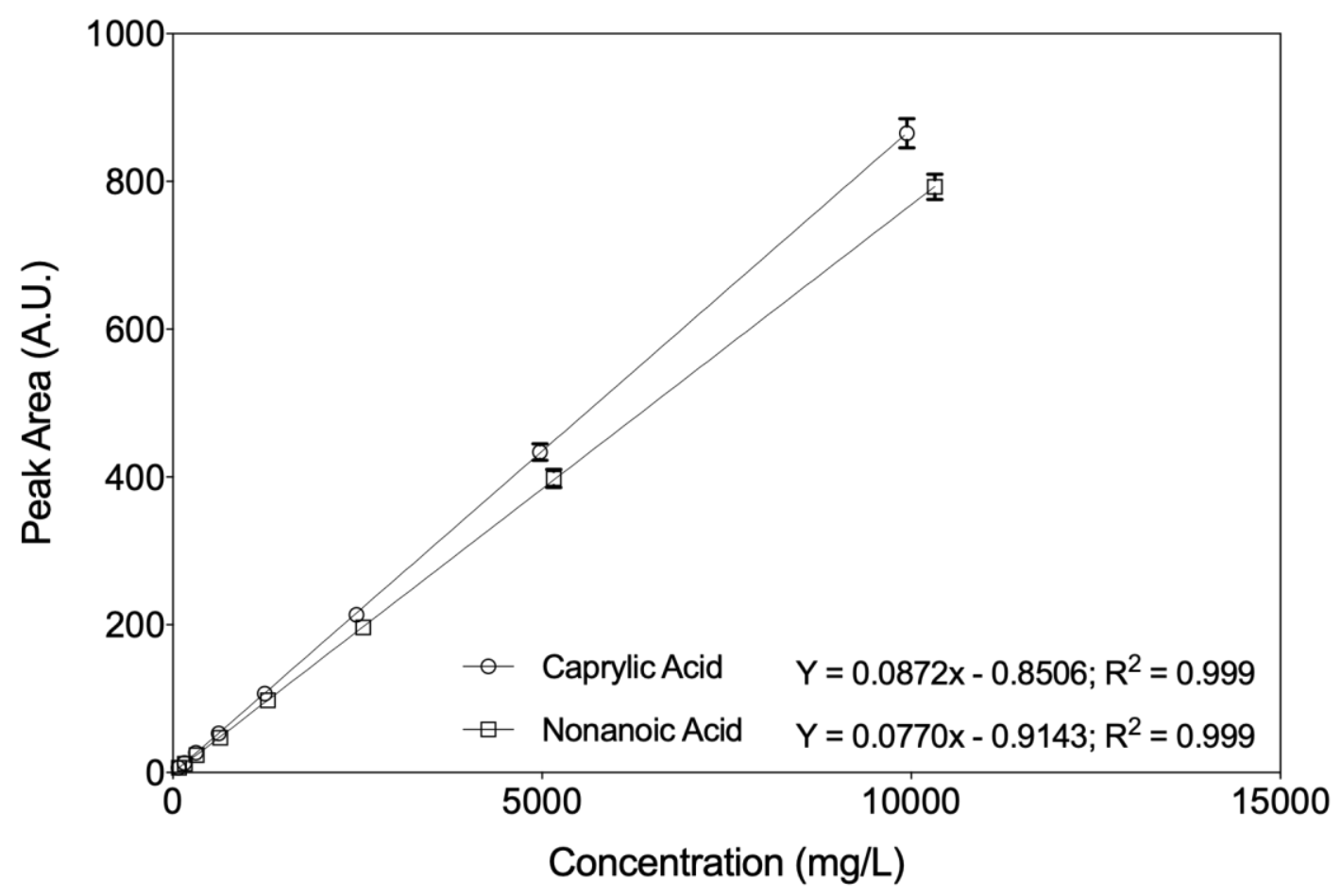

Figure S3. Calibration curves for nonanoic acid and caprylic acid in acetonitrile-water (60:40) over a concentration range of 78-10000 mg/L using HPLC-DAD with absorbance set to $210 \mathrm{~nm}$. 

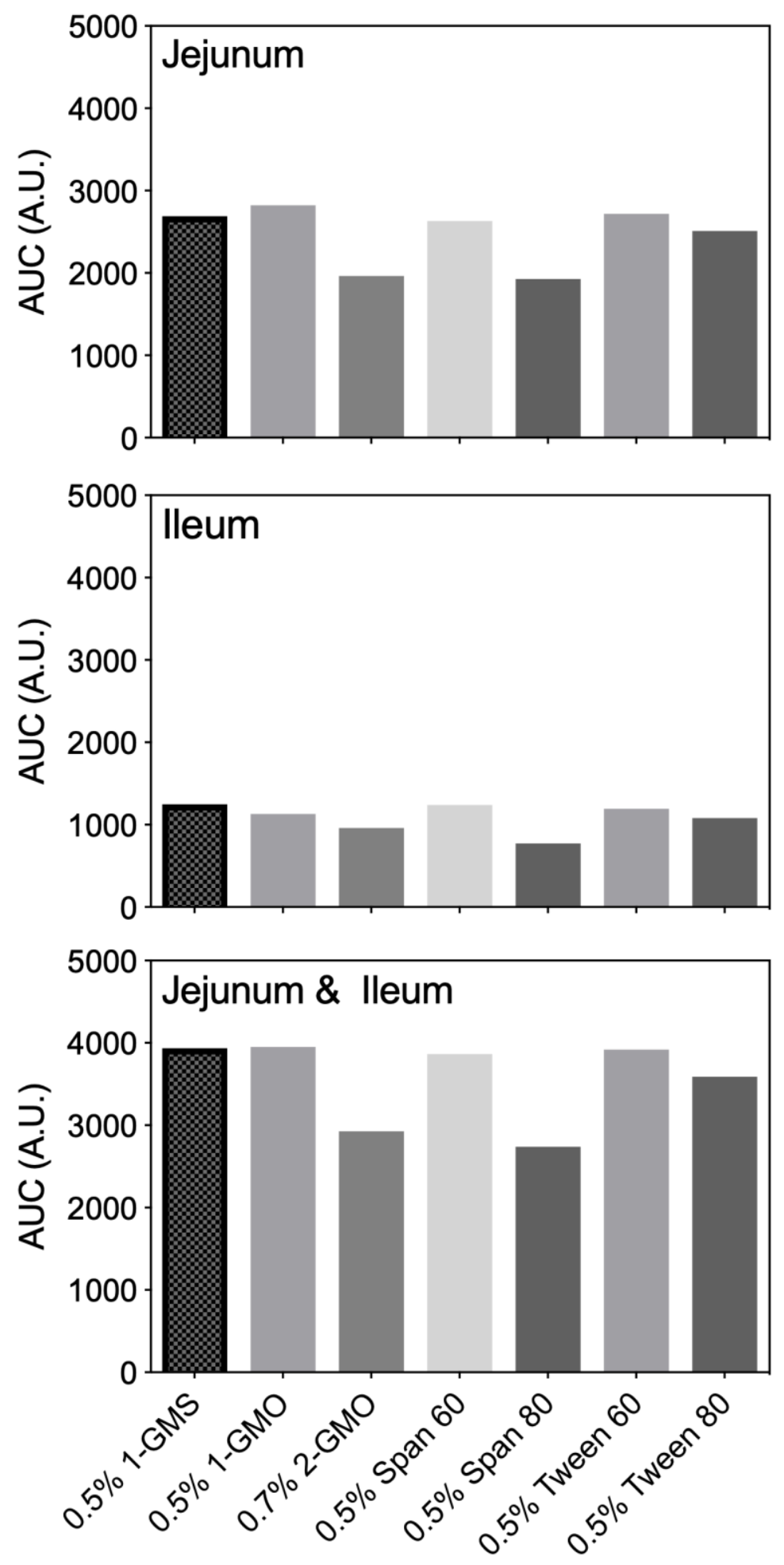

Figure S4. Area under the curve (AUC, expressed in A.U.) calculated from the absolute bioaccessibility values of caprylic acid over the $5 \mathrm{~h}$ digestion period using the trapezoid method. 


\section{Conclusions \& Future Works}

Surfactants are amphiphilic structures widely-used in the food industry as functional ingredients to facilitate the formation and stabilization of emulsions, as well as, increase product shelf-life. Surfactants can be divided into two major categories: low molecular weight surfactants (i.e., monoglycerides, Spans, Tweens, lecithins) and high molecular weight surfactants (i.e., proteins and polysaccharides). Numerous studies have established that food emulsion properties such as, choice of surfactant, characteristics of the oil phase, and emulsion droplet size, all have the potential to modify the rate and extent of lipid digestion. This study sought to further investigate the effects of surfactants on lipid digestion by comparing in vitro lipid digestion profiles of oil-in-water $(\mathrm{o} / \mathrm{w})$ emulsions stabilized with common low molecular weight surfactants (monoglycerides and non-ionic, synthetic surfactants) along with evaluating the impact of $s n$ position for monoglycerides.

Tricaprylin o/w emulsions were stabilized with one of the following surfactants: glycerol monostearate (1-GMS), glycerol monooleate (1-GMO), sn-2 glycerol monooleate (2-GMO), Span 60, Span 80, Tween 60 , or Tween 80 . Model test meals containing the prepared emulsions were fed into an advanced, multi-compartmental gastrointestinal model (TIM-1) for $5 \mathrm{~h}$ fed state digestion periods. There were no significant differences in time to reach effective lipolysis (i.e., $\mathrm{T}_{\mathrm{C}}$ ), interpreted as induction time, due to the frequency of the sampling intervals and preprogrammed transit time set for the TIM-1 system. Acid stability of surfactants had a limited role in modifying lipolysis rates which was attributed to surfactant concentration levels used (0.5-0.7\% w/w) and regulated gastric emptying time of the TIM-1 system. The impact of acid-stability is primarily observed in vivo by influencing gastric emptying rates; emulsion breakdown into aqueous and lipid layers causes the aqueous layer to empty the stomach more rapidly than the acid- 
stable meals. Both Span 80- and 2-GMO-stabilized o/w emulsions had statistically lower overall bioaccessibility compared to emulsions stabilized by the other surfactants. For Span 80-stabilized emulsions, this was attributed to the unsaturated oleic acid chain forming less stable expanded monolayers; thus, reducing emulsion stability (i.e., droplet coalescence) during digestion and decreasing available interfacial area for lipolytic activity. For 2-GMO-stabilized emulsions, this was attributed to an accumulation at the interface which hindered lipase adsorption and activity. The extent of lipolysis was expected to decrease in an $\mathrm{o} / \mathrm{w}$ emulsion system consisting of an interface covered by $s n-2$ monoglycerides which are resistant to enzymatic hydrolysis by $1 / 3$ regiospecific pancreatic lipase. No statistically significant differences were observed for overall bioaccessibility between emulsions stabilized with commercial 1-GMO and 1-GMS, Span 60, Tween 60, and Tween 80 . Overall, the results confirm that sn-position and surfactant choice have an evident impact on the extent lipid digestion in vitro.

The findings from this study provide insights into which common surfactants have the potential to modify lipid digestion. Further testing could involve emulsion stability tests during digestion by measuring particle size distributions throughout digestion. For example, sample measurements could be taken at intervals from the gastric compartment to evaluate the impact of acid stability of surfactants. Certain surfactants may be susceptible to hydrolysis by pancreatic lipase; thus, it may be of interest to correlate digestion of surfactants used to stabilize an o/w emulsion with lipid digestion kinetics of the o/w emulsion. As the TIM-1 uses semi-permeable capillary membranes to obtain bioaccessible fractions rather than bioavailability, testing in combination with intestinal cell lines or tissues (i.e., Caco-2 cells) could better model the human intestinal barrier. While careful inferences may be extrapolated from in vitro results, it is important that in vitro results are validated with in vivo studies. Clinical trials provide the opportunity to 
measure parameters such as post-prandial serum TAG levels, evaluate satiety responses, hormonal feedback mechanisms, and account for variability between individuals. Lastly, surfactants have been reported to affect gut microbiota composition and thus TIM-1 experiments could be paired with dynamic in vitro colon models (i.e., TIM-2, SHIME) to gain a more holistic understanding of the physiological effects of surfactants used to stabilize o/w emulsions.

The implications of this study for the food industry are to better understand the role of surfactants in lipid digestion, and eventually expand upon these findings to aid in the design and formulation of functional foods with tailored lipemic responses. Span 80 is a commercially available surfactant that merits further testing to evaluate its potential to control lipolysis, which could also potentially increase satiety responses in vivo. Sn-2 monoglycerides are not commonly used in food products; however, understanding the effects of lipolytic products generated in situ offers insights into the regulation of fat metabolism and implications, particularly for individuals who suffer from absorption insufficiencies. In conclusion, controlling interfacial composition by choice of surfactant has the potential to significantly influence the metabolic response of foods we consume. Ultimately, the goal of this project and further studies is to decrease the negative impacts of processed foods, provide consumers with healthier food product options, and better combat the rise in diet-related diseases. 